\title{
LACEwING: A New Moving Group Analysis Code
}

\author{
Adric R. Riedel ${ }^{1,2,3,4}$, Sarah C. Blunt ${ }^{4,5}$, Erini L. Lambrides ${ }^{6}$, Emily L. Rice ${ }^{2,4,7}$, \\ Kelle L. Cruz ${ }^{3,4,7}$, and Jacqueline K. Faherty ${ }^{4,8}$ \\ ${ }^{1}$ Department of Astronomy, California Institute of Technology, Pasadena, CA 91125, USA; arr@astro.caltech.edu \\ ${ }^{2}$ Department of Engineering Science and Physics, The College of Staten Island, Staten Island, NY 10314, USA \\ ${ }^{3}$ Department of Physics and Astronomy, Hunter College, New York, NY 10065, USA \\ ${ }^{4}$ Department of Astrophysics, American Museum of Natural History, New York, NY 10024, USA \\ ${ }^{5}$ Department of Physics, Brown University, Providence, RI 02912, USA \\ ${ }^{6}$ Department of Physics and Astronomy, Johns Hopkins University, Baltimore, MD 21218, USA \\ ${ }^{7}$ Physics Ph.D. Program, The Graduate Center, City University of New York, New York, NY 10016, USA \\ 8 Department of Terrestrial Magnetism, Carnegie Institute of Science, Washington DC 20015, USA \\ Received 2015 July 11; revised 2016 November 14; accepted 2016 November 15; published 2017 February 3
}

\begin{abstract}
We present a new nearby young moving group (NYMG) kinematic membership analysis code, LocAting Constituent mEmbers In Nearby Groups (LACEwING), a new Catalog of Suspected Nearby Young Stars, a new list of bona fide members of moving groups, and a kinematic traceback code. LACEwING is a convergence-style algorithm with carefully vetted membership statistics based on a large numerical simulation of the Solar Neighborhood. Given spatial and kinematic information on stars, LACEwING calculates membership probabilities in 13 NYMGs and three open clusters within $100 \mathrm{pc}$. In addition to describing the inputs, methods, and products of the code, we provide comparisons of LACEwING to other popular kinematic moving group membership identification codes. As a proof of concept, we use LACEwING to reconsider the membership of 930 stellar systems in the Solar Neighborhood (within $100 \mathrm{pc}$ ) that have reported measurable lithium equivalent widths. We quantify the evidence in support of a population of young stars not attached to any NYMGs, which is a possible sign of new as-yet-undiscovered groups or of a field population of young stars.
\end{abstract}

Key words: open clusters and associations: general - stars: kinematics and dynamics - stars: low-mass stars: pre-main sequence

Supporting material: animation, machine-readable table

\section{Introduction}

Young stars were traditionally thought to exist in starforming regions and open clusters, the closest of which are the Scorpius-Centaurus complex and Taurus-Auriga, both over $100 \mathrm{pc}$ away. In the last 30 years (starting with studies like Rucinski \& Krautter 1983 and de la Reza et al. 1989), a number of stars have been discovered within that distance that are relatively young $(5-500 \mathrm{Myr})$. This population of stars has been extensively studied and has immense scientific value as the nearest examples of later stages of star formation. Nearby young moving groups (NYMGs) are older than star-forming regions, but they are significantly closer and therefore their members are easier to study. As groups, the NYMGs are spread out over large (often overlapping) volumes of space and large areas of the sky, which makes defining groups and identifying interlopers challenging.

Currently, young stars within $100 \mathrm{pc}$ of the Sun and roughly 10 gravitationally unbound NYMGs (Table 1; Zuckerman \& Song 2004; Torres et al. 2008, p. 757; Malo et al. 2013) are thought to exist in three open clusters: Hyades, Coma Ber, and $\eta$ Cha. These moving groups (occasionally called "loose associations") are distinct from open clusters: they have no strong nuclei and are incredibly sparse, with a few dozen stars spread over thousands of cubic parsecs of space. They are also distinct from the streams and pre-Hipparcos kinematic overdensities like the Local Association/Pleiades Moving Group (Jeffries 1995; Montes et al. 2001b), Hyades Supercluster (Eggen 1985), and IC 2391 Supercluster (Eggen 1991), which have been identified as heterogeneous assemblages of stars
(Famaey et al. 2008). A few of the NYMGs appear to be related to open clusters: AB Dor to the Pleiades, Argus to IC 2391, and $\epsilon$ Cha to $\eta$ Cha, suggesting a common or at least related origin. The groups have ages between $\sim 5 \mathrm{Myr}$ old ( $\epsilon$ Cha) and 600-800 Myr old (Hyades).

The fundamental assumption about these NYMGs and open clusters is that they are the products of single bursts of star formation. This means that every constituent member should be roughly the same age (with attendant constraints on activity, radius, and rotational velocity), have the same chemical composition, have been in the same location at the time of formation, and have formed under the same conditions. Although the moving groups are not gravitationally bound, they are young enough that their space motion should still trace the Galactic orbits of their natal gas clouds. Due to their proximity and lack of gas, NYMG members allow easy and uncomplicated analysis of their photometric and spectroscopic properties.

The existence of these groups has been beneficial to the study of extremely low-mass objects-planets (Baines et al. 2012; Delorme et al. 2013), brown dwarfs (Faherty et al. 2016), and very low-mass stars (Mathieu et al. 2007, p. 411) - whose formation and evolutionary sequence and properties are still largely unknown. Using the assumption of a common origin, the age, metallicity, and formation environment deduced from the high-mass members can be applied to very low-mass objects.

The methods for identifying young stars vary with their mass and age. They include measurements of coronal activity, as seen in X-rays (Schmitt et al. 1995; Micela et al. 1999; 
Table 1

Nearby Young Moving Groups and Open Clusters

\begin{tabular}{|c|c|c|c|c|c|c|c|}
\hline \multirow{2}{*}{$\begin{array}{l}\text { Name } \\
\text { (1) }\end{array}$} & \multirow{2}{*}{$\begin{array}{l}\text { Abbreviation } \\
\text { (2) }\end{array}$} & \multicolumn{2}{|c|}{ Members } & \multicolumn{2}{|r|}{ Min Age } & \multicolumn{2}{|r|}{ Max Age } \\
\hline & & $\begin{array}{l}\text { All } \\
(3)\end{array}$ & $\begin{array}{c}\text { OBAFGK } \\
\text { (4) }\end{array}$ & $\begin{array}{c}\text { (Myr) } \\
(5)\end{array}$ & $\begin{array}{l}\text { Reference } \\
\text { (6) }\end{array}$ & $\begin{array}{l}\text { (Myr) } \\
(7)\end{array}$ & $\begin{array}{l}\text { Reference } \\
\text { (8) }\end{array}$ \\
\hline$\epsilon$ Chamæleontis & $\epsilon$ Cha & 35 & 17 & 5 & Murphy et al. (2013) & 8 & Torres et al. (2008, p. 757) \\
\hline$\eta$ Chamæleontis $^{\mathrm{a}}$ & $\eta$ Cha & 21 & 6 & 6 & Torres et al. (2008, p. 757) & 11 & Bell et al. (2015) \\
\hline TW Hydrae & TW Hya & 38 & 7 & 3 & Weinberger et al. (2013) & 15 & Weinberger et al. (2013) \\
\hline$\beta$ Pictoris & $\beta \mathrm{Pic}$ & 94 & 34 & 10 & Torres et al. (2008, p. 757) & 24 & Bell et al. (2015) \\
\hline 32 Orionis & 32 Ori & 16 & 12 & 15 & E. E. Mamajek (2016, private communication) & 65 & David \& Hillenbrand (2015) \\
\hline Octans & Octans & 46 & 22 & 20 & Torres et al. (2008, p. 757) & 40 & Murphy \& Lawson (2015) \\
\hline Tucana-Horologium & Tuc-Hor & 209 & 63 & 30 & Torres et al. (2008, p. 757) & 45 & Kraus et al. (2014a) \\
\hline Columba & Columba & 82 & 52 & 30 & Torres et al. (2008, p. 757) & 42 & Bell et al. (2015) \\
\hline Carina & Carina & 32 & 22 & 30 & Torres et al. (2008, p. 757) & 45 & Bell et al. (2015) \\
\hline Argus & Argus & 90 & 38 & 35 & Barrado y Navascués et al. (1999a) & 50 & Barrado y Navascués et al. (1999a) \\
\hline AB Doradus & AB Dor & 146 & 86 & 50 & Torres et al. (2008, p. 757) & 150 & Bell et al. (2015) \\
\hline Carina-Near & Car-Near & 13 & 10 & 150 & Zuckerman et al. (2006) & 250 & Zuckerman et al. (2006) \\
\hline Coma Berenices $^{\mathrm{a}}$ & Coma Ber & 195 & 104 & 400 & Casewell et al. (2006) & & \\
\hline Ursa Major & Ursa Major & 62 & 55 & 300 & Soderblom \& Mayor (1993) & 500 & King et al. (2003) \\
\hline$\chi^{01}$ Fornax & $\chi^{01}$ For & 14 & 14 & 500 & Pöhnl \& Paunzen (2010) & & \\
\hline Hyades $^{\mathrm{a}}$ & Hyades & 724 & 260 & 600 & Zuckerman \& Song (2004) & 800 & Brandt \& Huang (2015) \\
\hline
\end{tabular}

Notes. More details on these groups can be found in Section 7.

a Open cluster.

Feigelson et al. 2002; Torres et al. 2008, p. 757); UV activity (Shkolnik et al. 2012; Rodriguez et al. 2013); chromospheric activity, as seen in $\mathrm{H} \alpha$ (West et al. 2008) and optical calcium (Hillenbrand et al. 2013); measuring the lithium equivalent width (EW) (e.g., Mentuch et al. 2008; Malo et al. 2014a), EWs of gravity-sensitive spectral features (e.g., Lyo et al. 2004; Schlieder et al. 2012a); rotational velocity measurements (e.g., Mamajek \& Hillenbrand 2008); emission line core widths (e.g., Shkolnik et al. 2009); chemical abundances (e.g., D'Orazi et al. 2012; Tabernero et al. 2012; De Silva et al. 2013); and isochrone fitting (e.g., Torres et al. 2008, p. 757; Malo et al. 2013). Most of those techniques can establish or at least constrain ages for ranges of stellar temperatures and masses, but they cannot generally be used to identify memberships in a particular group. Conversely, kinematic memberships themselves do not generally convey any proof of youth (LópezSantiago et al. 2009), but they alone can group stars so that collective properties can be determined. Given that the spatial distributions of many NYMGs are overlapping and distributed across the sky (at least three-AB Dor, $\beta$ Pic, Ursa Major-are effectively all sky), space velocities are often the only practical way to identify memberships. This makes identifying members of NYMGs a different task from identifying members of more distant clusters, which are more localized on the sky.

A variety of codes are publicly available to accomplish the task of identifying NYMG members kinematically: BANYAN (Malo et al. 2013), which implements Bayesian methods to choose between membership in seven moving groups and a field/old option; BANYAN II (Gagné et al. 2014b), a modification of BANYAN with updated kinematic models and algorithms; and a convergence algorithm (Rodriguez et al. 2013) that uses the convergence points of the NYMGs to determine probable membership in six NYMGs. There are also other prominent but less widely available codes, including ones used in Montes et al. (2001b) and subsequent papers; Torres et al. (2008, p. 757) and subsequent papers, Kraus et al. (2014a), Lépine \& Simon (2009) and follow-up papers
(Schlieder et al. 2010, 2012b, 2012c), Shkolnik et al. (2012), Klutsch et al. (2014), Riedel et al. (2014), and Binks et al. (2015). All require varying amounts of the six kinematic elements to identify objects by their kinematics, but none include all the moving groups and open clusters currently believed to exist within 100 parsecs of the Sun. Throughout the paper, we will follow this nomenclature for the six kinematic elements: right ascension $\alpha \equiv$ R.A., declination $\delta \equiv$ decl., parallax $\pi$ (or distance Dist), proper motion in right ascension $\mu_{\alpha} \equiv \mu_{\text {R.A. }}$ cos decl., proper motion in declination $\mu_{\delta} \equiv \mu_{\text {decl. }}$, and systemic radial velocity $\gamma \equiv \mathrm{RV}$.

In this paper we present a new kinematic moving group code, LocAting Constituent mEmbers In Nearby Groups (LACEwING), which has been presented in international conferences (Riedel 2016b) and publications (Faherty et al. 2016; Riedel et al. 2016b). LACEwING, given stellar kinematic properties, calculates membership probabilities for 16 groups, comprising the 13 moving groups and 3 open clusters within $100 \mathrm{pc}$ mentioned earlier and in Table 1 . The following discussion, and the moving group code described herein, present three interrelated products:

1. The LACEwING kinematic moving group identification code (Section 2), comprised of both the code itself (Section 2.1) and its calibration (Section 2.2).

2. An epicyclic traceback code, TRACEwING (Section 3), which was used to identify interlopers in samples of NYMG members.

3. A catalog (Section 4) of data on 5350 known and suspected nearby young stars, from which a sample of 400 high-confidence members of NYMGs (later reduced to 297 systems; Section 5.1) and a sample of 930 lithiumdetected objects (Section 5.4) were taken. That new bona fide sample is used to form the kinematic models and calibration of the particular implementation of LACEwING presented here. 
As a proof of concept, we use LACEwING to calculate the membership probabilities for both the bona fide (Section 5.1) and lithium (Section 5.4) samples. We then compare LACEwING's recovery of known members in the lithium sample to BANYAN, BANYAN II, and the Rodriguez et al. (2013) convergent point analysis (Section 6), and outline our conclusions about the moving group members themselves (Section 7). Conclusions are summarized in Section 8.

\section{The LACEwING Moving Group Identification Code}

LACEwING is a frequentist observation space kinematic moving group identification code. Using the spatial and kinematic information available about a target object $(\alpha, \delta$, Dist, $\mu_{\alpha}, \mu_{\delta}$, and $\gamma$ ), it determines the probability that the object is a member of each of the known NYMGs (Table 1). As with other moving group identification codes, LACEwING is capable of estimating memberships for stars with incomplete kinematic and spatial information.

LACEwING works in right-handed Cartesian Galactic coordinates, with $U V W$ space velocities and $X Y Z$ space positions, where the $U / X$ axis is toward the Galactic center. The matrices of Johnson \& Soderblom (1987) transform observational equatorial coordinates into $U V W$ and $X Y Z$, and vice versa. The LACEwING code takes kinematic models of the NYMGs (represented as freely oriented triaxial ellipsoids in $U V W$ and $X Y Z$ space), predicts the observable values for members of each group at the $\alpha$ and $\delta$ of the input star, and compares them directly to the measured observable quantities.

Producing a membership probability from the goodness-offit value is a complex task, because most of the NYMGs considered here (particularly those under $100 \mathrm{Myr}$ ) are found within a very small range of space velocities, typified by the Zuckerman \& Song (2004) "good box," with boundaries $U=(0,-15) \mathrm{km} \mathrm{s}^{-1}, V=(-10,-34) \mathrm{km} \mathrm{s}^{-1}, W=(+3$, $-20) \mathrm{km} \mathrm{s}^{-1}$. Young stars are also significantly less common than older field stars (see Section 5.2.2). Thus, the potential for confusion between the groups and field is potentially large, and different for each NYMG. The LACEwING code accounts for these factors with equations, derived from a large simulation of the Solar Neighborhood, that convert goodness-of-fit parameters to membership probabilities.

A functional implementation of LACEwING requires three components:

1. A code to predict proper motion, radial velocity, and distances based on $\alpha$ and $\delta$; compare the predictions to input stellar data; and produce goodness-of-fit values, described in Section 2.1.

2. Per-NYMG equations to transform goodness-of-fit values into membership probabilities. The simulation of the Solar Neighborhood and method for producing the equations are given in Section 2.2.

3. Spatial and velocity distributions that describe each of the moving groups, used for predictions and calibration. The process of creating and vetting these stars is explained in Section 5.1, and uses data from the Catalog of Suspected Nearby Young Stars described in Section 4.

\subsection{The LACEwING Algorithm}

Within the LACEwING framework, each NYMG is represented by two triaxial ellipsoids described by base values
$U V W$ and $X Y Z$, semimajor and semiminor axes $A B C$ and $D E F$, and Tait-Bryant angles $U V, U W, V W, X Y, X Z$, and $Y Z$, which are used to rotate the ellipses around the $W, V, U$ and $Z, Y, X$ axes, respectively. This is unlike the BANYAN II (Gagné et al. 2014b) rotation order $U, V, W(X, Y, Z)$.

For each NYMG, we use 100,000 Monte Carlo iterations within the triaxial ellipsoids to generate a realistic spread of $U V W$ velocities. Using inverted matrices from Johnson \& Soderblom (1987), we convert the $U V W$ velocities into $\mu_{\alpha}, \mu_{\delta}$, and $\gamma$ for a simulated star at the $\alpha$ and $\delta$ of the target, at a standard distance, which we have chosen to be $10 \mathrm{pc}$ in analogy to the absolute magnitude. The lengths of the proper motion vectors are later used to estimate the kinematic distance, which can be compared to a parallax measurement if it exists.

As an example, we use the bona fide $\beta$ Pic member AO Men (K4 Ve). At the $\alpha$ and $\delta$ of AO Men and a distance of $10 \mathrm{pc}$, an object sharing the mean $U V W$ velocity of $\beta$ Pic should have $\mu_{\alpha}=-45 \operatorname{mas~yr}^{-1}, \mu_{\delta}=289$ mas yr $^{-1}$, and $\gamma=+16 \mathrm{~km} \mathrm{~s}^{-1}$.

With the predicted values of $\mu_{\alpha}, \mu_{\delta}$, and $\gamma$, and at least some measured kinematic data on the star, the code derives (up to) four of the following metrics:

1. Proper motion. The code splits the measured proper motion into components parallel $\left(\mu_{\|}\right)$and perpendicular $\left(\mu_{\perp}\right)$ to the predicted proper motion vector; the best match is where the perpendicular component is zero. Our goodness-of-fit metric for proper motions is

$$
\psi_{\mu}^{2}=\frac{\mu_{\perp \text { star }}^{2}}{\sigma_{\mu_{\perp \text { star }}}^{2}+\sigma_{\mu_{\perp \text { pred }}}^{2}} .
$$

If $\mu_{\| \text {star }}$ has the opposite sign from $\mu_{\| \text {pred }}$ and is more than $1 \sigma$ from zero, we add 1000 to $\psi_{\mu}$ to ensure a poor goodness-of-fit metric.

For our example object AO Men, the real proper

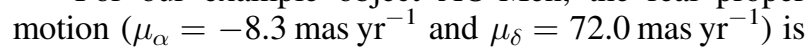
along a vector very similar to the predicted object,

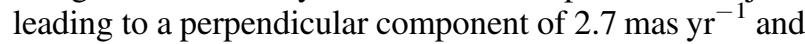
a $\psi_{\mu}$ of $0.05 \sigma$.

2. Distance. Kinematic distance is derived from a ratio of the star's measured proper motion and the predicted proper motion calculated for a member at $10 \mathrm{pc}$ :

$$
\frac{\text { Dist }_{\text {pred }}}{10}=\frac{\mu_{\text {para,pred }}}{\mu_{\text {para,star }}} .
$$

If a trigonometric parallax distance exists, the resulting goodness-of-fit metric is

$$
\psi_{\text {Dist }}^{2}=\frac{\text { Dist }_{\text {star }}^{2}-\text { Dist }_{\text {pred }}^{2}}{\sigma_{\text {Dist, star }}^{2}+\sigma_{\text {Dist,pred }}^{2}} .
$$

For our example object AO Men, the two parallel components of the proper motion are 72.4 mas yr $^{-1}$ (real)

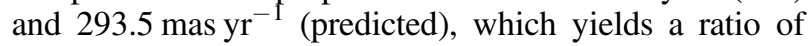
4.05 and a distance of $40.5 \mathrm{pc}$. The actual distance (measured by Hipparcos; van Leeuwen 2007-we are not using the Gaia DR1 results from Gaia Collaboration et al. 2016) is 38.6 pc.

3 . Radial velocity. The code compares the measured radial velocity to the predicted $\gamma$. In this case, the goodness-of- 
fit metric is

$$
\psi_{\gamma}^{2}=\frac{\gamma_{\text {star }}^{2}-\gamma_{\text {pred }}^{2}}{\sigma_{\gamma, \text { star }}^{2}+\sigma_{\gamma, \text { pred }}^{2}}
$$

For AO Men, the predicted $\gamma$ of an ideal member of $\beta$ Pic is +16.04 , while the measured $\gamma$ of AO Men is +16.25 . The difference between the two is $0.1 \sigma$.

4. Position. With either trigonometric parallax or kinematic distance, the code uses the $\alpha, \delta$ and distance to determine how near the star is to the moving group or cluster. As the moving groups are defined with freely oriented ellipses, this requires a matrix rotation to bring a 100,000 element Monte Carlo approximation of the stellar position uncertainty into the coordinate system of the moving group. The goodness-of-fit metric is

$$
\psi_{\text {Pos }}^{2}=\frac{X_{\text {star }}^{2}}{\sigma_{X}^{2}+\sigma_{D}^{2}}+\frac{Y_{\text {star }}^{2}}{\sigma_{Y}^{2}+\sigma_{E}^{2}}+\frac{Z_{\mathrm{star}}^{2}}{\sigma_{Z}^{2}+\sigma_{F}^{2}} .
$$

AO Men and its measured parallax put it $30 \mathrm{pc}(2.1 \sigma)$ from the center of the $\beta$ Pic moving group.

While the last test for spatial position is not a standard convergence test, it is useful for preventing the code from identifying members of spatially concentrated groups (e.g., Hyades, TW Hya) in physically unreasonable locations.

Each of the four goodness-of-fit metrics has a different characteristic median value when analyzing bona fide members, and that value varies slightly between groups. The median $\psi_{\mu}$ value is particularly small ( $\left.\sim 0.02\right)$ when matching bona fide members to their correct groups, while $\psi_{\text {Dist }}$ and $\psi_{\gamma}$ are much larger $(\sim 0.5)$, and $\psi_{\text {Pos }}$ was generally around 2 . All goodnessof-fit values tended to be significantly larger when matching stars to the wrong groups, although $\psi_{\mu}$ was again the most sensitive discriminator.

\subsection{LACEwING Calibration}

At this stage, the LACEwING code has produced up to four different goodness-of-fit metrics estimating the quality of match between a star and one of the NYMGs. We combine these metrics in quadrature:

$$
\psi^{2} \times N_{\text {metrics }}=\psi_{\mu}^{2}+\psi_{\text {Dist }}^{2}+\psi_{\gamma}^{2}+\psi_{\text {Pos}}^{2}
$$

All tests indicated that the code recovers more members when each metric is given equal weight, as shown here.

We now want to derive functions to transform the goodnessof-fit values into membership probabilities. This is not simple, because the groups overlap with each other to different degrees. Some, like Ursa Major, have distinct $U V W$ velocities that do not overlap with any other group. Others, like the Hyades, have unique and compact $X Y Z$ spatial distributions. Most, however, overlap in $U V W$ and $X Y Z$ with other moving groups.

The additional complication is that there are seven different possible combinations of data, each with its own goodness-offit value that we need to calibrate separately, for each moving group considered:

$$
\begin{aligned}
& \text { 1. } \alpha, \delta, \mu_{\alpha} \text { and } \mu_{\delta} . \\
& \text { 2. } \alpha, \delta, \text { Dist. } \\
& \text { 3. } \alpha, \delta, \gamma \text {. } \\
& \text { 4. } \alpha, \delta, \mu_{\alpha} \text { and } \mu_{\delta}, \text { Dist. } \\
& \text { 5. } \alpha, \delta, \mu_{\alpha} \text { and } \mu_{\delta}, \gamma \text {. }
\end{aligned}
$$

6. $\alpha, \delta$, Dist, $\gamma$.

7. $\alpha, \delta, \mu_{\alpha}$ and $\mu_{\delta}$, Dist, $\gamma$.

To quantify these probabilities, we generate a simulation of the Solar Neighborhood that we run through the LACEwING algorithm. The results of the simulation will be used to determine the relationship between the goodness of fit and membership probability.

When generating the simulation, a random number generator first assigns the new star to one of the groups, in proportion to the population of each group as derived in Section 5.1. Thus, more field stars will be generated than TW Hya members because the field is more populous.

To generate $U V W$ and $X Y Z$ values for a simulated star, we use the freely oriented ellipse parameters of the moving groups (from Section 5.1). If the assigned group is one of the NYMGs, a $U V W$ velocity and $X Y Z$ position is generated under the assumption that the velocity and spatial distributions are both Gaussian. If the assigned group is the field, the $U V W$ velocities are assumed to be Gaussian distributions, but the $X Y$ positions are drawn from a uniform distribution truncated at semimajor and semiminor axes $D$ and $E$, and the $Z$ position above the plane is drawn from an exponential distribution with a scale height of 300 parsecs (Bochanski et al. 2010) truncated at semiminor axes $F$

Once a star has been generated, the $U V W$ and $X Y Z$ values are converted into equatorial coordinates $\alpha, \delta, \pi, \mu_{\alpha}, \mu_{\delta}$, and $\gamma$. Measurement errors are generated in the form of Gaussian

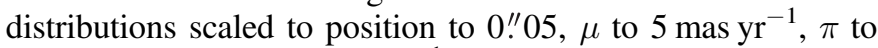
0.5 mas, and $\gamma$ to $0.5 \mathrm{~km} \mathrm{~s}^{-1}$, and added to the equatorial values. The simulated stars are then run through the LACEwING algorithm, comparing them to every moving group using all seven combinations of data. The goodness-of-fit values are then recorded.

To produce the actual relations that yield membership percentages, we then take all of the stars compared to a particular moving group $X$, using a combination of data $Y$. The goodness-of-fit values are binned into 0.1 goodness-of-fit value bins. Within each bin, we calculate the fraction of stars in that bin that were actually generated as members ("true" members) of moving group $X$.

These functions are best described by Gaussian Cumulative Distribution Functions (CDFs) with normalized amplitude. The fraction of stars that were "true members" as a function of goodness-of-fit value (for $\beta$ Pic) is shown in Figure 1 as histograms, along with the fitted functions. The coefficients of the Gaussian CDFs are saved and used to derive membership probabilities given a goodness-of-fit value.

In order to handle the case where a star is already known to be young (and the probability of membership in an NYMG is higher), there is a second calibration of LACEwING. In this mode, a subset containing all the NYMG members and an equally sized portion of the field stars (to account for the young field; see Section 8) is retained, for a 1:1 ratio of NYMG members:young field is selected. This subset is binned into 0.1 goodness-of-fit bins, fractions of stars are calculated in the same way as the above "field star" sample, and a different set of Gaussian CDF coefficients is produced (the "young star" curves for $\beta$ Pic are shown in Figure 2).

These curves are different for different moving groups; the results of Coma Ber in Field Star mode are shown in Figure 3. With 16 groups, 7 combinations of data, and 2 modes of 


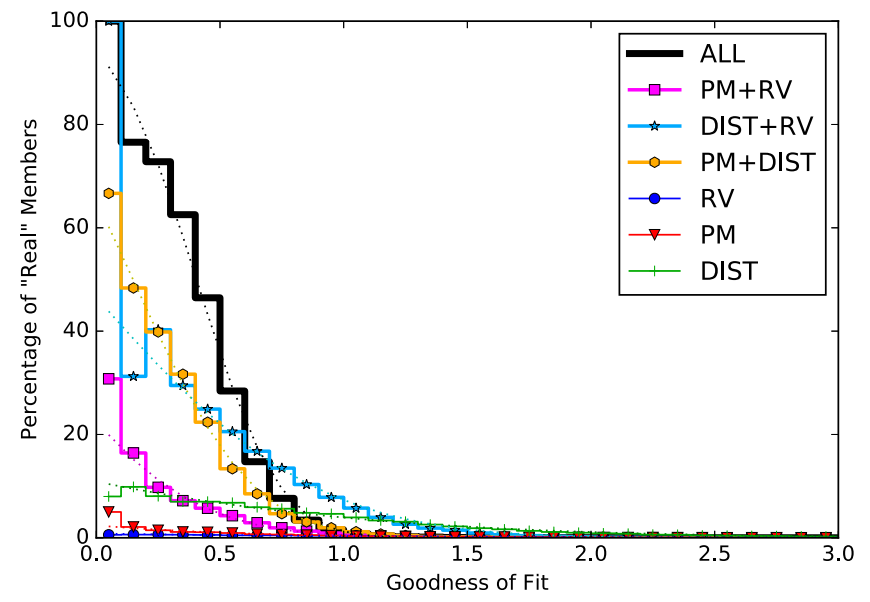

Figure 1. Goodness-of-fit histograms for $\beta$ Pic in field star mode and associated fitted Gaussian CDFs (curved lines). Higher curves mean that the estimated probability of membership in $\beta$ Pic is greater.

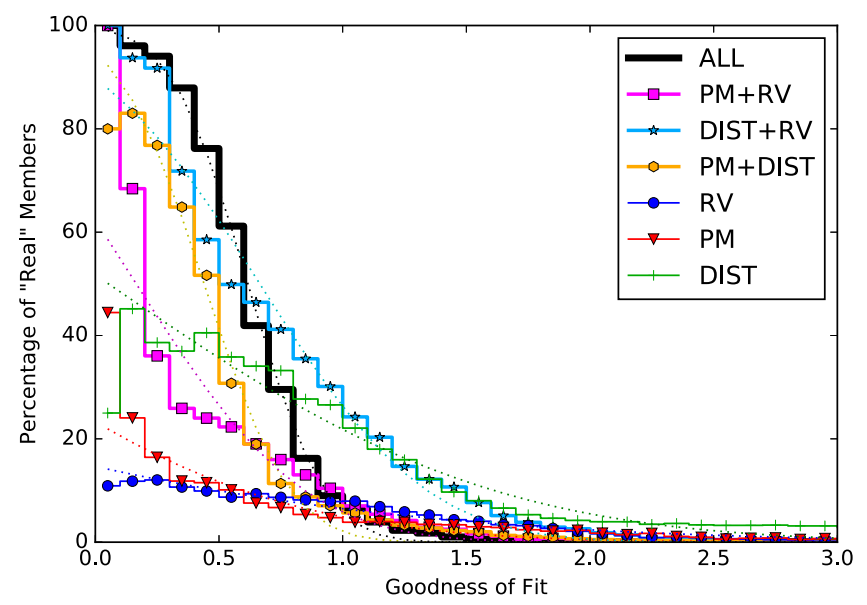

Figure 2. Same as Figure 1, but for $\beta$ Pic in young star mode.

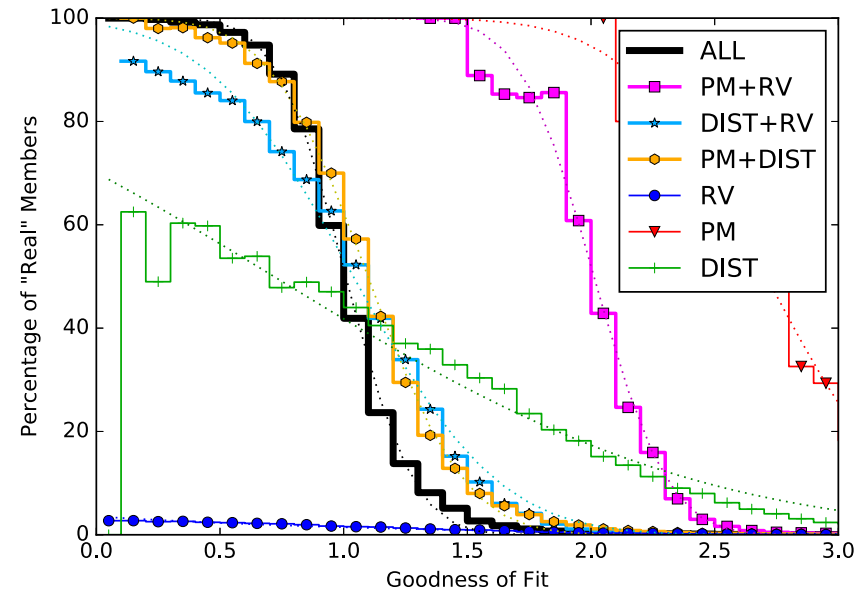

Figure 3. Same as Figure 1, but for Coma Ber. Coma Ber has a unique space velocity and spatial position that allows us to identify members more confidently.

operation, there are 224 sets of coefficients that make up this implementation of LACEwING.

For AO Men, our combined goodness-of-fit value was 0.52 . In the field star mode histogram (Figure 1) and the case of having complete kinematic data, 3632 of the 8 million stars

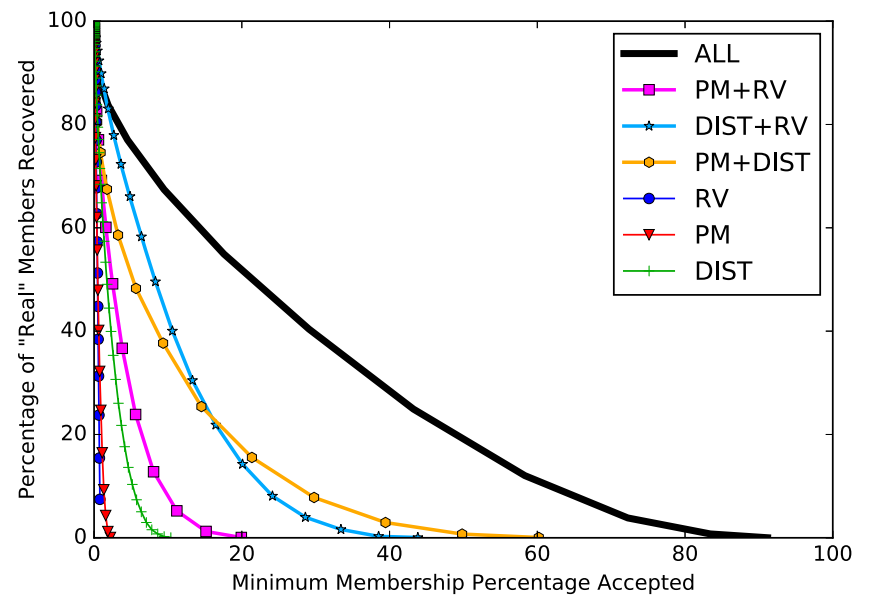

Figure 4. Percentage of "true" members of $\beta$ Pic (from the simulation) recovered as a function of minimum acceptable membership probability.

scored between 0.5 and 0.6 , and only 1032 of those (28\%) were generated as members of $\beta$ Pic. If we use the young star mode histograms (Figure 2), 61\% of the stars in the 0.5-0.6 bin were genuine members of $\beta$ Pic. Using the actual curve fits to compute the probabilities of $\beta$ Pic membership for AO Men yields $26 \%$ (field star mode) and $57 \%$ (young star mode), with an additional $3 \%$ (field star) $/ 13 \%$ (young star) chance that it is actually a member of Columba according to those curves.

The membership probabilities given by LACEwING are ultimately the complement of the contamination probability: the probability that a star is a member of a given group and not something else. This is a subtly different question than, "what group $X$ is star $Z$ a member of?" The latter question involves comparing different NYMGs directly, and is much more difficult to answer. When interpreting LACEwING probabilities, it is important to keep in mind that LACEwING does not force all membership probabilities to add up to $100 \%$ in the way that BANYAN and BANYAN II do; each of the probabilities of membership is an independent assessment of "Group $X$ or not Group $X$ ?" connected only by the fact that all the probability coefficients are derived from the same simulation. Probabilities indicated by LACEwING may add up to more than $100 \%$ if the uncertainties on the input parameters are larger than the typical values in the simulation. In practice, taking the NYMG that is matched with the highest membership probability is an excellent means of identifying memberships.

It is important to generate enough simulated stars to properly sample the membership function for even the smallest groupa minimum of roughly 1000 stars are necessary to fit the Gaussian CDF correctly. For the particular implementation of LACEwING presented in this paper, the smallest group was $\eta$ Cha (6 members, out of 40,902), which required at least 6.8 million simulated stars; 8 million were calculated.

From Figure 1, in field star mode and with all kinematic data, even the best possible match to $\beta$ Pic (goodness-of-fit $=0$ ) has only a $92 \%$ probability of membership in $\beta$ Pic. That is, $8 \%$ of objects that match $\beta$ Pic perfectly are not members. With only proper motion, the maximum possible probability of membership in $\beta$ Pic is only 5\%. The young star mode yields higher probabilities; Figure 2 shows a maximum probability of $100 \%$ for $\beta$ Pic members with all information, and a maximum membership probability of $23 \%$ for only proper motion. If we plot the cumulative number of $\beta$ Pic members recovered as a 


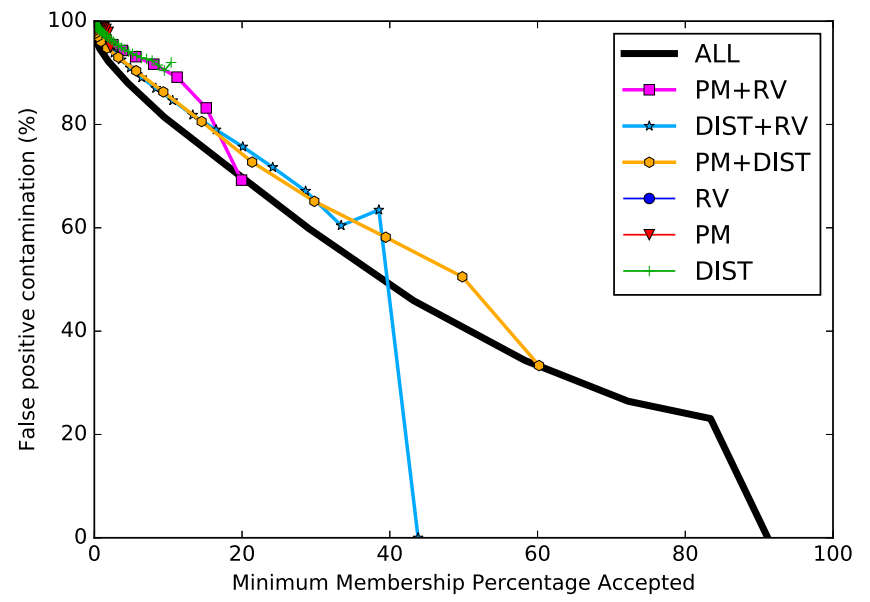

Figure 5. Percentage of false positives in a data set as a function of the minimum accepted membership percentage in $\beta$ Pic.

function of minimum probability accepted (Figure 4), we see that we have to set a minimum threshold of $10 \%$ membership probability to recover $90 \%$ of the members in the best possible case where all kinematic data are available.

Setting a low membership probability cutoff means a much larger false positive contamination, as shown in Figure 5. Selecting an adequate cutoff requires balancing the recovery rate with the contamination rate (Figure 6; similar figures describing the BANYAN II Bayesian models can be found in Figures 5 and 6 of Gagné et al. 2014b). The false positive rates highlight the danger of using kinematics alone to identify young stars: any kinematically selected survey needs to use other spectroscopic and photometric youth indicators to weed out false positives.

Rough guidelines used throughout the rest of this work, and in the stand-alone version of LACEwING, are that probabilities of $66 \%$ and higher are high probabilities of membership, $40 \%-$ $66 \%$ and above are moderate probability, $20 \%-40 \%$ are low probability, and below $20 \%$ is too low to consider meaningful.

Technical details on using LACEwING, recalibrating LACEwING, and incorporating it into other codes are given in Appendix A.

\section{The TRACEwING Epicyclic Traceback Code}

What we wish to accomplish with tracebacks is to identify and reject stars that could not possibly have been near the formation site of a moving group at the time of formation. They may still fall within the $U V W$ velocity (and even $X Y Z$ spatial) dispersion of the current distribution, but if we track their position as a function of time they will end up far from the rest of the members.

\subsection{Principles of Traceback}

The TRACEwING code uses an epicyclic approximation of Galactic orbital motion (Makarov et al. 2004) to trace the positions of stars back in time using their current measured motion, in increments of $0.1 \mathrm{Myr}$. It compares the positions of single objects to an NYMG, which is represented by stored freely oriented ellipse parameters fit using the same process used in Section 2.1. Based on the equal-volume radius of the group, the TRACEwING code presents a single-valued representation of how close the target is to the moving group as a function of time.

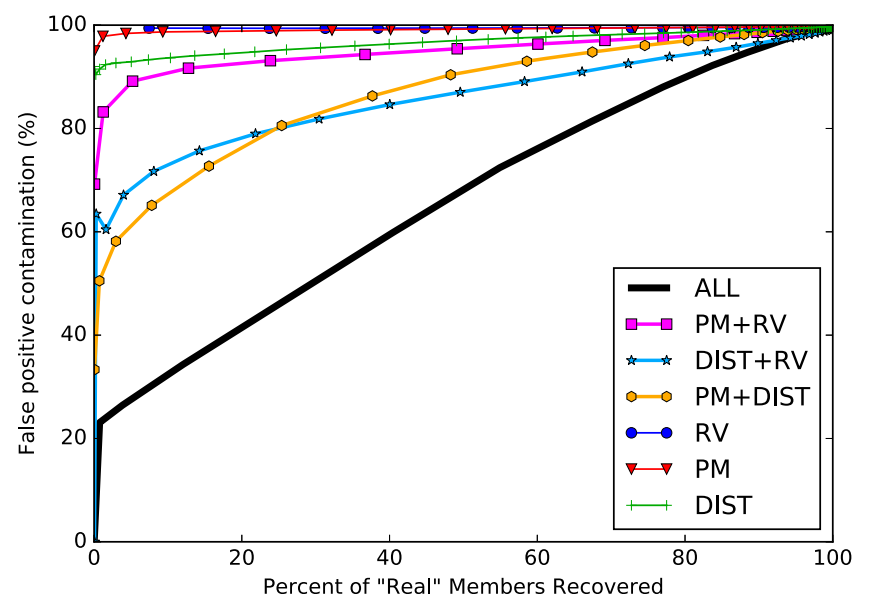

Figure 6. $\beta$ Pic recovery rate of LACEwING as a function of the false positive rate.

TRACEwING is essentially two separate steps, carried out by two different programs:

1. A program that uses the epicyclic kinematic approximation to trace all bona fide members of an NYMG back in time, and fits freely oriented ellipsoids to the ensemble at each time step, saving the parameters for future use.

2. A program that uses the epicyclic kinematic approximation to trace a single star back in time, and compares its positions to saved moving group ellipsoids at each time step.

\subsection{Design of TRACEwING}

With epicyclic traceback, the effects of Galactic orbital motion are approximated by use of sine and cosine functions, controlled by Oort constants and a vertical oscillation parameter. For TRACEwING, we use the equations of position (relative to the Sun, as a function of time in Myr) given in Makarov et al. (2004) and reproduced here:

$$
X=X_{0}+U_{0} \kappa^{-1} \sin \kappa T+\left(V_{0}-2 A X_{0}\right)(1-\cos \kappa T)(2 B)^{-1},
$$

$$
\begin{aligned}
Y= & Y_{0}-U_{0}(1-\cos \kappa T)(2 B)^{-1} \\
& +V_{0}(A \kappa T-(A-B) \sin \kappa T)(\kappa B)^{-1} \\
- & 2 X_{0} A(A-B)(\kappa T-\sin \kappa T)(\kappa B)^{-1}, \\
& Z=Z_{0} \cos \nu T+W_{0} \nu^{-1} \sin \nu T .
\end{aligned}
$$

In the above equations, $A$ and $B$ are the Oort constants (from Bobylev 2010; $A=+0.0178 \mathrm{~km} \mathrm{~s}^{-1} \mathrm{pc}^{-1}, B=-0.0132 \mathrm{~km} \mathrm{~s}^{-1}$ $\left.\mathrm{pc}^{-1}\right) ; \kappa$ is the planar oscillation frequency, $\sqrt{-4 B \times(A-B)}$; and $\nu$ is the vertical oscillation frequency, $2 \pi / 85 \mathrm{Myr}^{-1}$ (Makarov et al. 2004). The approximation deviates noticeably from an unperturbed linear traceback motion after 10 million years.

For the first step of TRACEwING, we calculate 4000 Monte Carlo tracebacks for each bona fide member, in time increments of 0.1 Myr. At each time step, freely oriented ellipses are fit to the positions of the members of the moving group for each of the 4000 Monte Carlo trials, and averaged to produce a mean position, extent, and orientation of the group at that time. These parameters are saved for comparison to individual stars. 


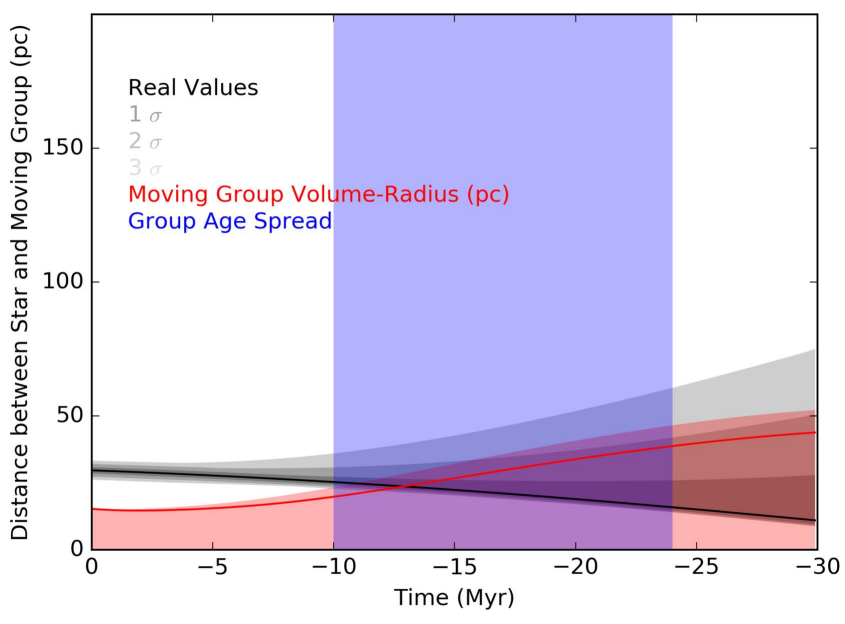

Figure 7. Separation between the star AO Men and the center of the $\beta$ Pic moving group (black line), as a function of time. The dark, medium, and light gray regions show the $1 \sigma, 2 \sigma$, and $3 \sigma$ uncertainties on the separation between AO Men and $\beta$ Pic. The red line and red region show the volume-equivalent radius of $\beta$ Pic itself (and its $1 \sigma$ uncertainty). The blue region represents the range of ages of $\beta$ Pic. AO Men's kinematics (black line and dark gray region) place it plausibly within the radius of $\beta$ Pic (red region) at the time of formation (blue region).

For the second step, we take a target object of interest, which may be any object with full kinematic information-star, brown dwarf, or planet. To determine the potential memberships of the target object, we generate and trace back 20,000 trials within each of the $1 \sigma, 2 \sigma$, and $3 \sigma$ uncertainties on their observational (equatorial) positions and motions. At each time step, the distance between the object and the previously calculated position of the moving group (both in Cartesian Galactic $X Y Z$ coordinates) is calculated, and an equal-volume radius $\left(\frac{4}{3} \pi r^{3}=\frac{4}{3} \pi a b c\right)$ is used as the effective radius of the group. The targets are then visually classified by whether the $1 \sigma, 2 \sigma$, or $3 \sigma$ positions potentially place them within the effective radius of the group at the time of formation. The traceback of the bona fide $\beta$ Pic member AO Men is displayed in Figure 7, and shows a star that was plausibly within the confines of $\beta$ Pic at the time of formation.

\subsection{Traceback Limitations}

Epicyclic approximations do not take into account the gravitational influence of other stars, molecular clouds, Gould's Belt, or the Galactic disk and bar itself. This is most pronounced in open clusters, where the stars themselves are gravitationally bound to each other, but sets limits on the reliability of the technique for moving groups as well.

To quantify the limits of the technique, we perform two tests. First, we simulate a "real" moving group of stars, move it forward in time, "observe" it, and trace it back in time to see what a genuine NYMG of various ages should look like traced back to its origin. Second, we select unrelated field stars with a velocity and spatial distribution similar to known NYMGs, and move it back in time as a "fake" NYMG. The upper limit on reliability of the traceback technique is the point at which the "real" moving group is indistinguishable in volume from the "fake" one.

Our "real" moving group is a simulated group of 50 stars with a Gaussian velocity dispersion of $1.5 \mathrm{~km} \mathrm{~s}^{-1}$ (Preibisch \& Mamajek 2008) typical of the Sco-Cen star-forming region, and a uniform spatial distribution with a radius of $5 \mathrm{pc}$. These values are perhaps smaller than most clusters, but provide a best-case scenario. The 50 stars were moved forward in time in steps of $0.1 \mathrm{Myr}$ using the epicyclic approximation and their positions (with the mean position subtracted off, so all stars were near the origin at the final epoch) were saved at 5, 8, 12, $25,45,50,125,250,400$, and 500 Myr intervals. To observe the stars, we generated new $U V W$ velocities using the individual stars' change of position over the last $0.1 \mathrm{Myr}$ of the simulated time range, e.g.,

$$
U=0.9778 \frac{\mathrm{km} \mathrm{Myr}}{\mathrm{s}^{-1} \mathrm{pc}} \Delta X \frac{\mathrm{pc}}{0.1 \mathrm{Myr}} .
$$

We then converted all $U V W X Y Z$ values to equatorial coordinates, and applied randomly generated "observational"

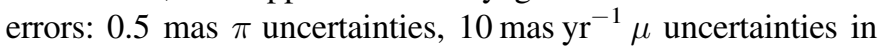
each axis, and $1 \mathrm{~km} \mathrm{~s}^{-1} \gamma$ uncertainties. These collections of stars were run back in time the same way as before to determine the apparent size at formation. To test the trivial case (perfect information), we added no observational errors to the generated cluster of stars and traced them back in time.

To assemble the group of field stars, we searched the Extended Hipparcos catalog of Anderson \& Francis (2012) to find stars with $\mu, \pi$, and $\gamma$ distributed according to the presentday median velocity dispersion and spatial distribution parameters of our unbound moving groups, with a velocity dispersion of $1.6 \mathrm{~km} \mathrm{~s}^{-1}$ and spatial distribution within $11.5 \mathrm{pc}$. Our fake moving group is centered on $X Y Z=(-5,-5,20) \mathrm{pc}$ and $U V W=(-5,-5,-5) \mathrm{km} \mathrm{s}^{-1}$, a well-populated region of velocity space not populated by any known NYMG. The 15 selected stars are given in Table 2.

In the trivial case with no observational uncertainties (Figure 8), the synthetic group traces back to having its smallest volume (although it is larger than the $5 \mathrm{pc}$ initial radius) near the actual time of formation. In the more realistic case (Figure 9), the large measurement errors mean that the minimum size of the simulated moving group appears to be right now, and the apparent effective radius of the simulated moving group at time of formation is $40 \mathrm{pc}$. In Figure 10 we plot the estimated volume-at-formation of the simulated moving group at various ages, and the fake moving group of field stars moved back in time.

In the case of the fake moving group made up of unrelated field stars (Figure 11), the volume is smaller than the simulated moving group (Figure 10) after $125 \mathrm{Myr}$. The epicyclic approximation's Galactic shear causes the simulated group to have a larger present-day velocity dispersion than is currently expected for the known NYMGs, suggesting that we are missing outlying members of the known groups. Crucially, these stars should trace back toward the origin of the moving group as they do in our simulated moving group example, unlike the outliers we intend to remove from current membership lists.

The Gaia mission will provide astrometry that is several magnitudes more precise than is currently available for stars brighter than $G \approx 20.7$. To determine the effectiveness of Gaia data, we have repeated our first test using measurement uncertainties expected for $G \sim 15$ sources (Gaia Collaboration et al. 2016) of $50 \mu$ as for parallaxes, $40 \mu$ as for positions, and $25 \mu \mathrm{as} \mathrm{yr}^{-1}$ for each axis of proper motions. The simulated Gaia-precision data demonstrate significant improvements. The size of the simulated burst of stars shrinks by a factor of 1000 (roughly linearly with the increased precision). As late as 
Table 2

Stars in the Fake Cluster

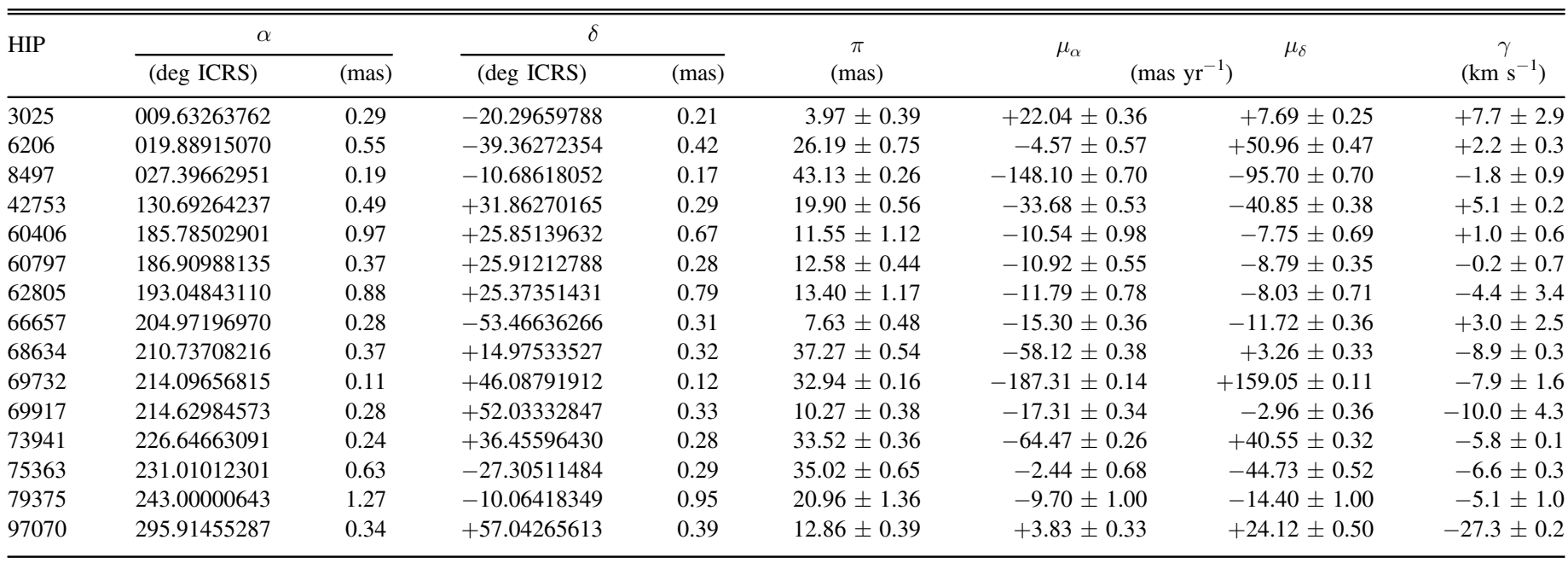

Note. All stars in the Extended Hipparcos Catalog (Anderson \& Francis 2012) with full kinematic information within $11.5 \mathrm{pc}$ of $X Y Z=(-5,-5,20)$ pc and $1.6 \mathrm{~km} \mathrm{~s}^{-1}$ of $U V W=(-5,-5,-5) \mathrm{km} \mathrm{s}^{-1}$.

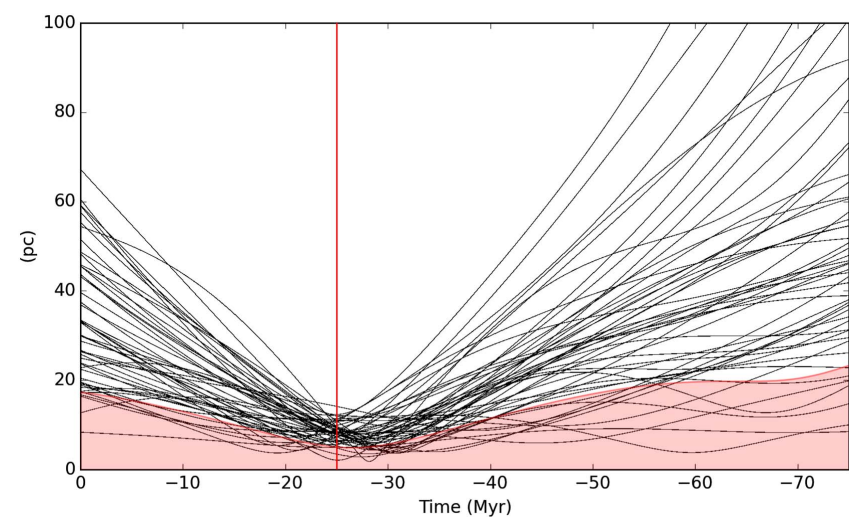

Figure 8. Separations between generated "real" stars moved forward $25 \mathrm{Myr}$ and the center of their simulated moving group when traced back, as a function of time. The $1 \sigma$ volume equivalent radius, representing the volume of the ellipse fit to the calculated positions of the bona fide member draws, is represented by the red curve; the time of formation is shown by the vertical red line.

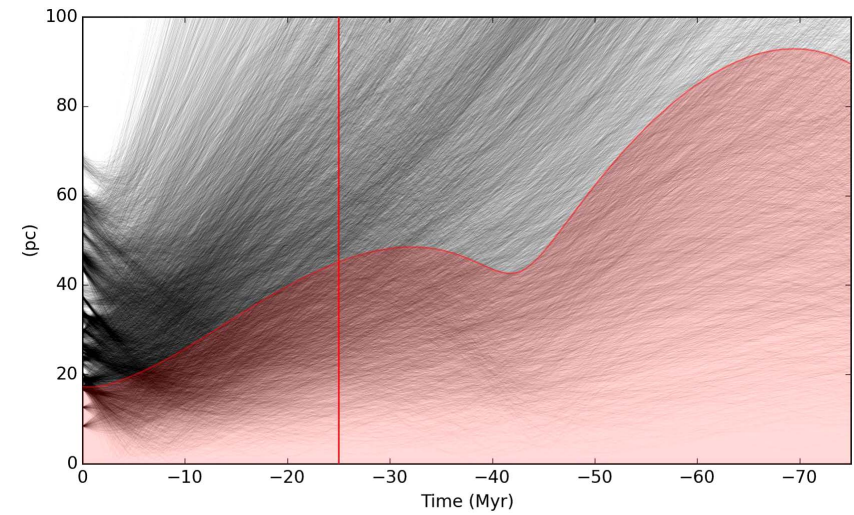

Figure 9. Same as Figure 8, but with realistic measurement errors added to the sample before tracing the stars back to their position $25 \mathrm{Myr}$ in the past. The stars no longer converge to a small effective radius in the past, and do not exhibit any kind of minimum at or near the time of formation $25 \mathrm{Myr}$ ago.
$25 \mathrm{Myr}$ (Figure 12), there is still a minimum (although not the correct radius) in the spatial distribution of the stars near the actual age of the group, and the radius is now relatively constant over time.

We can conclude a few things about the efficacy of tracebacks from these tests, presented here in no particular order:

1. The effectiveness of tracebacks is almost entirely limited by the current measurement precision of the parameters $\alpha, \delta, \pi, \mu$, and $\gamma$. After roughly $125 \mathrm{Myr}$, the cumulative effects of measurement uncertainties make it impossible to distinguish between a group of stars spreading out from a single point of origin and an unphysical collection of field stars on roughly parallel tracks. We cannot, therefore, comment on the existence of any moving groups older than $125 \mathrm{Myr}$ based on epicyclic tracebacks alone. As measurement uncertainties shrink, we will gradually approach the "perfect" case (Figure 8) where genuinely related stars will trace back to volumes smaller than unrelated stars selected with a similar velocity dispersion.

2. We cannot say anything about the membership of objects that trace back to within the boundary of the moving group at the time of formation-with the currently available measurement precision they could be true members or coincidental field stars. However, stars that do not trace back to possibly be within the confines are a different case. We found that only $6 \%$ of simulated members in our $45 \mathrm{Myr}$ old sample were not plausibly within the boundary of the moving group (at the $1 \sigma$ positional uncertainty level) $45 \mathrm{Myr}$ in the past. In contrast, far more than $6 \%$ of actual moving group members (Section 5.1) did not trace back to within the confines of their purported moving group, suggesting that the objects are not real members of the group. These are easily identifiable nonmembers, and as data precision improves, we expect to find more of these objects. 

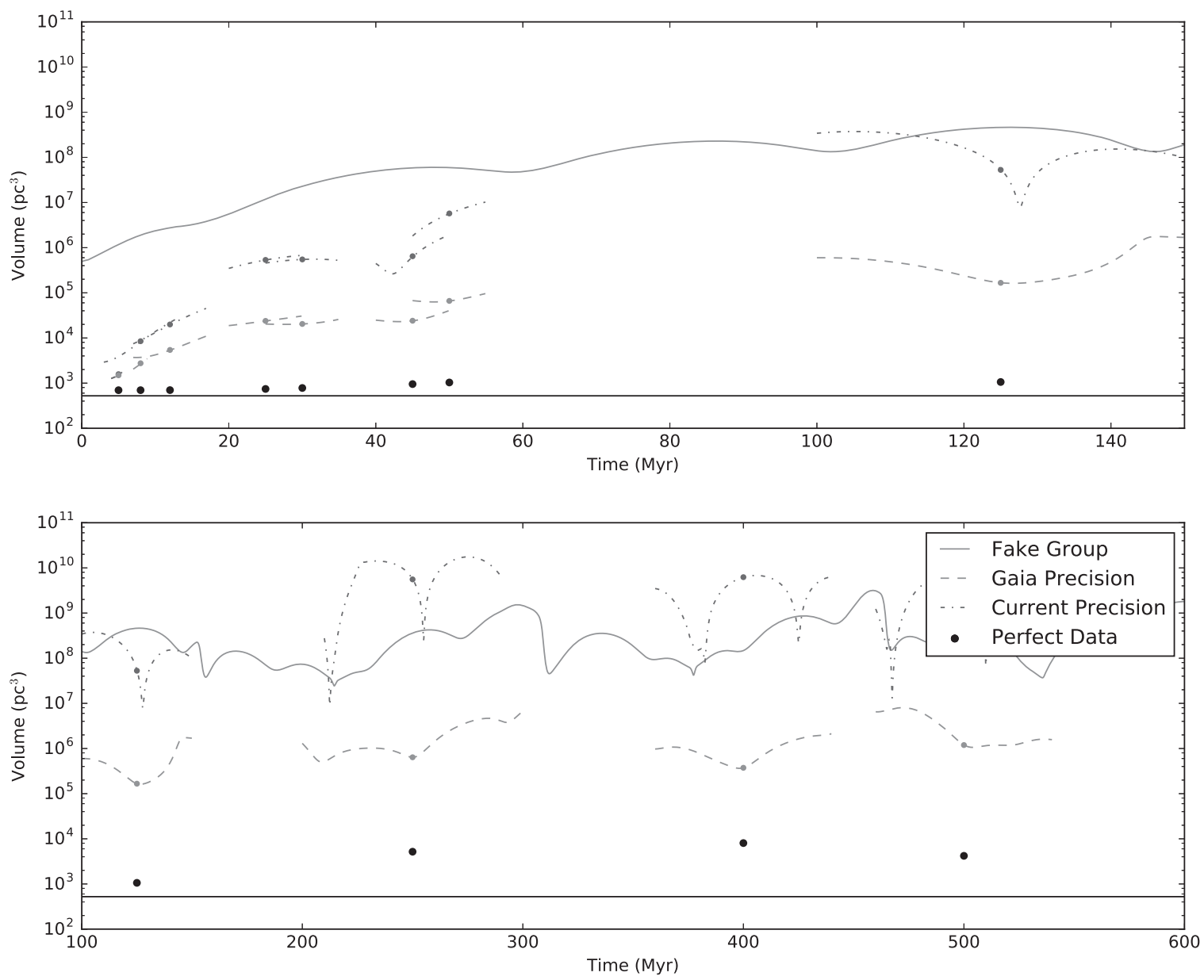

Figure 10. Demonstration of the volume-at-formation of our simulated moving group as a function of time-since-formation and observational precision: top panel, from 0 to $150 \mathrm{Myr}$; bottom panel, from 100 to $600 \mathrm{Myr}$. The cases with current-precision kinematics are shown with dashed-dotted lines surrounding the formation time of the group. The Gaia-precision cases are shown with dashed lines. For perfect data (no errors), the volumes at the times of formation are shown as black points. The fake moving group of field stars is shown as a solid curve.

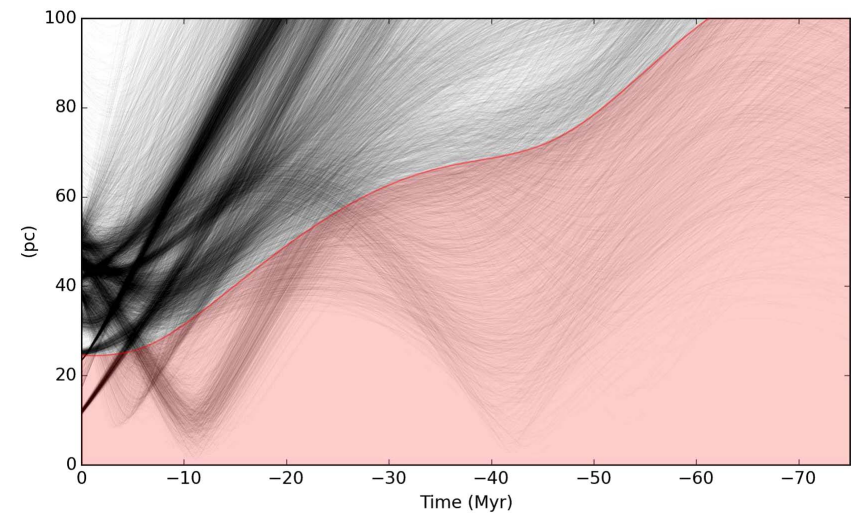

Figure 11. Same as Figure 9, but showing the results of our selected "fake" sample of field stars when they are traced back in time.

3. Very few of the stars in the fake moving group are consistent with possibly being in the center of the moving group (or really, anywhere except the edges of the group), while in the simulated moving group, nearly all the members are consistent with being in the center of the NYMG. This would seem to be one difference between an actual NYMG and an unphysical selection of stars.

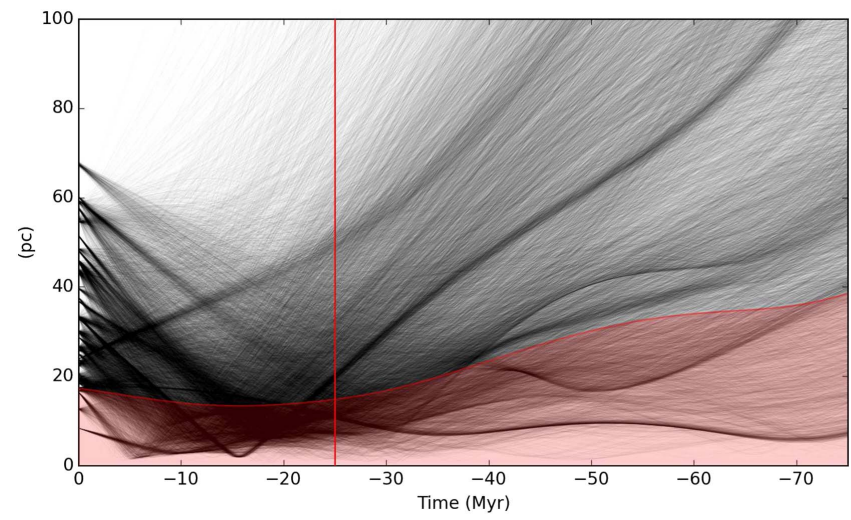

Figure 12. Same as Figure 9, but with measurement errors consistent with Gaia astrometry for Gaiamag $\sim 15$ stars.

\section{The Catalog of Suspected Young Stars}

Calibrating and testing LACEwING requires kinematic information on genuine members. For this implementation of LACEwING, the bona fide sample (Section 5.1) and proof-ofconcept lithium sample (Section 5.4) come from a catalog of all known and suspected young stars maintained by the authors.

The catalog is intended to contain basic information on every star, planetary-mass object, and brown dwarf in all nearby 


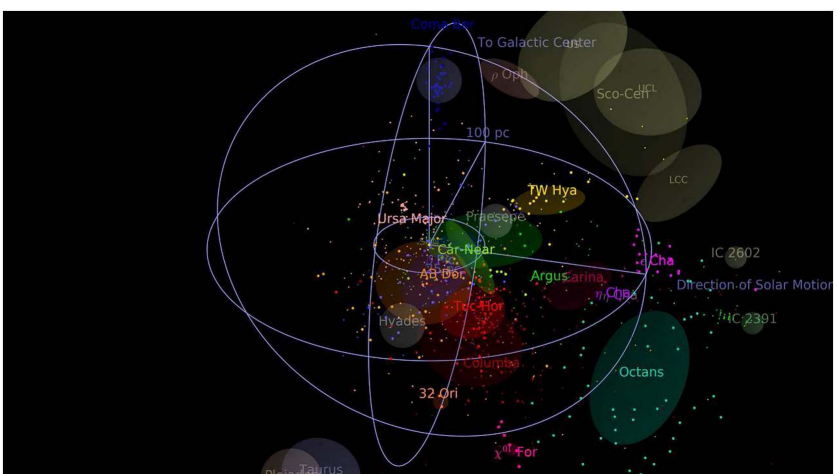

Figure 13. 3D representation of the positions of all NYMG stars in the Catalog of Suspected Nearby Young Stars with either parallaxes or kinematic distances, as seen from a vantage point at $\alpha=200, \delta=+20$, Dist $=200 \mathrm{pc}$. Ellipsoids of the moving groups, and the final bona fide members, shown as larger points, are taken from Section 5.1. Rough positions of other open clusters and starforming regions within $\sim 200 \mathrm{pc}$ are shown for scale and orientation only.

(An animation of this figure is available.)

(Dist $<100 \mathrm{pc}$ ) star systems ever reported as young, to provide a single resource for studying the individual and ensemble properties of young stars. It currently contains 5350 objects. Through careful literature searches, this catalog can reasonably be considered complete for membership in the NYMGs published through 2015 January. A similar effort has not been made for the Hyades and Coma Ber open clusters, and they cannot be considered complete, nor does the catalog necessarily contain all information known about the young targets. An animated 3D representation of the catalog is shown in Figure 13 plotted in $X Y Z$ coordinates.

As the table is quite large, a list of its headers is given in Table 3 with the full machine-readable table linked in the HTML version of this article. Catalogue updates are available in $\mathrm{GitHub}^{9}$ in CDS format, a comma-separated value file, an OpenDocument Spreadsheet, and an Office Open XML spreadsheet with the archival versions preserved at Zenodo (Riedel et al. 2016a). The catalog was constructed from a wide variety of source papers that reported young stars, identified through a literature search and from other papers collecting data sets (Zuckerman et al. 2013; Alonso-Floriano et al. 2015). The full list is given in Table 4.

It is assumed that the relevant results of earlier papers not on this list (e.g., de la Reza et al. 1989; Kastner et al. 1997; Webb et al. 1999; Torres et al. 2000, 2003; Song et al. 2002) have been superceded by or included in the more recent papers on the NYMGs (Table 4).

Within the catalog, 1312 of the 5350 total objects have never been reported as members of any group, including groups not believed to be real (Castor), classical pre-Hipparcos moving groups like the Local Association, or more distant groups like Upper Centaurus Lupus or Chamæleon I. These nonmembers fall into four categories:

1. Objects with ambiguous membership that match multiple groups equally well, as reported by Moór et al. (2006), Malo et al. (2013, 2014a, 2014b), and Gagné et al. (2014b).

2. Young objects that do not match any known group, as reported by Shkolnik et al. (2009, 2011), Maldonado

$\overline{9 \text { https://github.com/ ariedel/young_catalog }}$

Table 3

Headers of the Catalog of Nearby Suspected Young Stars

\begin{tabular}{|c|c|c|c|}
\hline Number & Label & Units & Description \\
\hline 1 & RAh & $\mathrm{hr}$ & $\begin{array}{l}\text { R.A. Hours (J2000 E2000) } \\
\text { (calculated) }\end{array}$ \\
\hline 2 & RAm & $\operatorname{arcmin}$ & $\begin{array}{l}\text { R.A. Minutes (J2000 E2000) } \\
\text { (calculated) }\end{array}$ \\
\hline 3 & RAs & $\operatorname{arcsec}$ & $\begin{array}{l}\text { R.A. Seconds (J2000 E2000) } \\
\quad \text { (calculated) }\end{array}$ \\
\hline 4 & DE- & $\cdots$ & $\begin{array}{l}\text { Decl. sign (J2000 E2000) } \\
\text { (calculated) }\end{array}$ \\
\hline 5 & DEd & $\operatorname{deg}$ & $\begin{array}{l}\text { Decl. Degrees (J2000 E2000) } \\
\text { (calculated) }\end{array}$ \\
\hline 6 & DEm & $\operatorname{arcmin}$ & $\begin{array}{l}\text { Decl. Minutes (J2000 E2000) } \\
\text { (calculated) }\end{array}$ \\
\hline 7 & DEs & $\operatorname{arcsec}$ & $\begin{array}{l}\text { Decl. Seconds (J2000 E2000) } \\
\quad \text { (calculated) }\end{array}$ \\
\hline 8 & Seq & $\ldots$ & Sequence Number \\
\hline 9 & LiSample & $\cdots$ & $\begin{array}{l}\text { [LIFfAa] Lithium Sample } \\
\text { Flag. (1) }\end{array}$ \\
\hline 10 & Bonafide & $\cdots$ & $\begin{array}{l}\text { [BbRrXx] Bona fide Sample } \\
\text { Flag. (2) }\end{array}$ \\
\hline 11 & MultStars & $\cdots$ & $\begin{array}{l}\text { ? Number of objects in system } \\
\text { ( } 0 \text { if object is a secondary). } \\
\text { Blank if unknown }\end{array}$ \\
\hline 12 & sepkey & $\cdots$ & $\begin{array}{l}\text { Key for system separation } \\
\text { considered here }\end{array}$ \\
\hline 13 & MultType & $\cdots$ & Type of multiplicity (3) \\
\hline 14 & Sep & $\operatorname{arcsec}$ & separation in arcseconds \\
\hline 15 & SepPA & $\operatorname{deg}$ & $\begin{array}{l}\text { ? Last known position angle of } \\
\text { separation. }\end{array}$ \\
\hline 16 & SepDate & year & ? Date separation was recorded \\
\hline 17 & orbper & $\ldots$ & Orbital Period \\
\hline 18 & orbperunit & $\cdots$ & Orbital Period Unit $\left(\mathrm{d} \mathrm{yr}^{-1}\right)$ \\
\hline 19 & r_Sep & $\cdots$ & reference for Sep \\
\hline 20 & $\mathrm{dV}$ & mag & ? Delta magnitude \\
\hline 21 & dVFilter & $\cdots$ & Filter of delta magnitude \\
\hline 22 & r_dV & $\cdots$ & reference for $\mathrm{dV}$ \\
\hline 23 & Name & $\cdots$ & Common Name \\
\hline 24 & TYCHO-2 & $\cdots$ & TYCHO-2 Identifier (I/259) \\
\hline 25 & GJ & $\cdots$ & $\begin{array}{l}\text { Gliese-Jahreiss Catalog of } \\
\text { Nearby Stars ID (J/PASP/ } \\
122 / 885)\end{array}$ \\
\hline 26 & HD & $\cdots$ & $\begin{array}{l}\text { ? Henry Draper catalog ID } \\
\text { (III/135A) }\end{array}$ \\
\hline 27 & HR & $\cdots$ & $\begin{array}{l}\text { ? Bright Star Catalog ID } \\
(\mathrm{V} / 50)\end{array}$ \\
\hline 28 & $\mathrm{DM}$ & $\cdots$ & $\begin{array}{l}\text { Durchmustrung ID (I/122; I/ } \\
\quad 119 ; \mathrm{I} / 114 ; \mathrm{I} / 108)\end{array}$ \\
\hline 29 & $1 \mathrm{RXS}$ & $\cdots$ & $\begin{array}{l}\text { ROSAT All-Sky Survey ID } \\
\text { (IX/10A; IX/29) }\end{array}$ \\
\hline 30 & UCAC4 & $\cdots$ & $\begin{array}{l}\text { Fourth USNO CCD Astro- } \\
\text { graphic Catalog ID } \\
\text { (I/322A) }\end{array}$ \\
\hline 31 & PPMXL & $\cdots$ & PPMXL ID (I/317) \\
\hline 32 & 2MASS & $\cdots$ & $\begin{array}{l}\text { Two Micron All Sky Survey } \\
\text { ID (II/246; II/281) }\end{array}$ \\
\hline 33 & SDSS & $\cdots$ & $\begin{array}{l}\text { Sloan Digital Sky Survey } \\
\text { Photometric Catalog ID } \\
\text { (V/139) }\end{array}$ \\
\hline 34 & ALLWISE & $\cdots$ & AllWISE ID (II/328) \\
\hline 35 & YPC & $\cdots$ & $\begin{array}{l}\text { ? General Catalog of Trigono- } \\
\text { metric Parallaxes ID } \\
\text { (I/238A) }\end{array}$ \\
\hline 36 & HIP & $\cdots$ & ? Hipparcos ID (I/311) \\
\hline 37 & RAdegraw & $\operatorname{deg}$ & Raw R.A. (J2000) \\
\hline 38 & e_RAdegraw & mas & uncertainty on RAdegraw \\
\hline 39 & DEdegraw & $\operatorname{deg}$ & Raw decl. (J2000) \\
\hline 40 & e_DEdegraw & mas & uncertainty on DEdegraw \\
\hline 41 & JD & $\mathrm{d}$ & \\
\hline
\end{tabular}


Table 3

Table 3

(Continued)

\begin{tabular}{|c|c|c|c|c|c|c|c|}
\hline Number & Label & Units & Description & Number & Label & Units & Description \\
\hline & & & Epoch of position & 87 & e_u'mag & mag & quality of u'mag \\
\hline & & & measurement & 88 & q_u'mag & $\cdots$ & reference for u'mag \\
\hline 42 & refPOS & $\cdots$ & Reference for position & 89 & r_u'mag & $\cdots$ & ? SDSS $g^{\prime}$ magnitude \\
\hline \multirow[t]{2}{*}{43} & RAdeg & deg & R.A. (J2000 E2000) & 90 & g'mag & mag & ? uncertainty on g'mag \\
\hline & & & (calculated) & 91 & e_g'mag & mag & quality of g'mag \\
\hline \multirow[t]{2}{*}{44} & e_RAdeg & mas & uncertainty on RAdeg & 92 & q_g'mag & $\cdots$ & reference for g'mag \\
\hline & & & (calculated) & 93 & r_g'mag & $\cdots$ & ? SDSS $r$ ' magnitude \\
\hline \multirow[t]{2}{*}{45} & DEdeg & deg & Decl. (J2000 E2000) & 94 & r'mag & mag & ? uncertainty on r'mag \\
\hline & & & (calculated) & 95 & e_r'mag & mag & quality of r'mag \\
\hline \multirow[t]{2}{*}{46} & e_DEdeg & mas & uncertainty on DEdeg & 96 & q_r'mag & $\ldots$ & reference for r'mag \\
\hline & & & (calculated) & 97 & r_r'mag & $\cdots$ & ? SDSS $i$ magnitude \\
\hline \multirow[t]{2}{*}{47} & plx & mas & ? Weighted Mean Parallax & 98 & i'mag & mag & ? uncertainty on i'mag \\
\hline & & & (calculated) & 99 & e_i'mag & mag & quality of i'mag \\
\hline \multirow[t]{2}{*}{48} & e_plx & mas & ? uncertainty on plx & 100 & q_i'mag & $\ldots$ & reference for i'mag \\
\hline & & & (calculated) & 101 & r_i'mag & $\cdots$ & ? SDSS $z$ magnitude \\
\hline \multirow[t]{2}{*}{49} & HRV & $\mathrm{km} \mathrm{s}^{-1}$ & ? Weighted Mean Heliocentric & 102 & z'mag & mag & ? uncertainty on $z$ 'mag \\
\hline & & & Radial Velocity (calculated) & 103 & e_z'mag & mag & quality of z'mag \\
\hline \multirow[t]{2}{*}{50} & e_HRV & $\mathrm{km} \mathrm{s}^{-1}$ & ? uncertainty on HRV & 104 & q_z'mag & $\cdots$ & reference for $z$ 'mag \\
\hline & & & (calculated) & 105 & r_z'mag & $\cdots$ & [JD] SDSS joint photometry \\
\hline 51 & PredDist & $\mathrm{pc}$ & ? Predicted Distance & & & & flag (4) \\
\hline 52 & e_PredDist & $\mathrm{pc}$ & ? uncertainty on PredDist & 106 & jointSDSS & $\cdots$ & ? Tycho-2 Bt magnitude \\
\hline 53 & PredDist-method & $\cdots$ & Method for PredDist & 107 & Btmag & mag & ? uncertainty on Btmag \\
\hline 54 & r_PredDist & $\cdots$ & Reference for predDist & 108 & e_Btmag & mag & ? Tycho-2 Vt magnitude \\
\hline \multirow[t]{2}{*}{55} & PredHRV & $\mathrm{km} \mathrm{s}^{-1}$ & ? Predicted Heliocentric Radial & 109 & Vtmag & mag & ? uncertainty on Vtmag \\
\hline & & & Velocity & 110 & e_Vtmag & mag & ? Johnson $B$ magnitude \\
\hline 56 & e_PredHRV & $\mathrm{km} \mathrm{s}^{-1}$ & ? uncertainty on PredHRV & 111 & Bmag & mag & ? uncertainty on Bmag \\
\hline 57 & PredHRV-method & $\cdots$ & Method for PredHRV & 112 & e_Bmag & mag & quality of Bmag \\
\hline \multirow[t]{2}{*}{58} & r_PredHRV & $\cdots$ & Reference for predicted Radial & 113 & q_Bmag & $\cdots$ & reference for Bmag \\
\hline & & & Velocity & 114 & r_Bmag & $\cdots$ & ? Johnson $V$ magnitude \\
\hline 59 & pmRA & mas $\mathrm{yr}^{-1}$ & $?$ pmRA $^{*} \cos ($ decl. $)$ & 115 & Vmag & mag & ? uncertainty on Vmag \\
\hline 60 & e_pmRA & mas $\mathrm{yr}^{-1}$ & ? uncertainty on pmRA & 116 & e_Vmag & mag & quality of Vmag \\
\hline 61 & $\mathrm{pmDE}$ & mas $\mathrm{yr}^{-1}$ & $? \mathrm{pmDEC}$ & 117 & q_Vmag & $\cdots$ & reference for Vmag \\
\hline 62 & e_pmDE & mas $\mathrm{yr}^{-1}$ & ? uncertainty on PMDE & 118 & r_Vmag & $\cdots$ & ? Cousins $R$ magnitude \\
\hline 63 & refPM & $\cdots$ & Reference for proper motion & 119 & Rmag & mag & ? uncertainty on Rmag \\
\hline \multirow[t]{2}{*}{64} & $\mathrm{pm}$ & mas $\mathrm{yr}^{-1}$ & Total proper motion & 120 & e_Rmag & mag & reference for Rmag \\
\hline & & & (calculated) & 121 & r_Rmag & $\cdots$ & ? Cousins $I$ magnitude \\
\hline 65 & e_pm & mas $\mathrm{yr}^{-1}$ & uncertainty on pm (calculated) & 122 & Imag & mag & ? uncertainty on Imag \\
\hline \multirow[t]{2}{*}{66} & PA & $\operatorname{deg}$ & Proper motion position angle & 123 & e_Imag & mag & quality of Imag \\
\hline & & & (calculated) & 124 & r_Imag & $\cdots$ & reference for Imag \\
\hline 67 & e_PA & $\operatorname{deg}$ & uncertainty on PA (calculated) & 125 & jointOpt & $\cdots$ & [JD] Optical joint photometry \\
\hline 68 & Uvel & $\mathrm{km} \mathrm{s}^{-1}$ & ? Galactic motion $U$ direction & & & & flag (4) \\
\hline & & & (toward Galactic center) & 126 & Jmag & mag & ? J magnitude \\
\hline 69 & e_Uvel & $\mathrm{km} \mathrm{s}^{-1}$ & ? uncertainty on Uvel & 127 & e_Jmag & mag & ? uncertainty on Jmag \\
\hline 70 & Vvel & $\mathrm{km} \mathrm{s}^{-1}$ & ? Galactic motion $V$ direction & 128 & q_Jmag & $\ldots$ & quality of Jmag \\
\hline & & & (toward solar motion) & 129 & Hmag & mag & ? $H$ magnitude \\
\hline 71 & e_Vvel & $\mathrm{km} \mathrm{s}^{-1}$ & ? uncertainty on Vvel & 130 & e_Hmag & mag & ? uncertainty on Hmag \\
\hline 72 & Wvel & $\mathrm{km} \mathrm{s}^{-1}$ & ? Galactic motion $W$ direction & 131 & q_Hmag & $\cdots$ & quality of Hmag \\
\hline & & & (toward Galactic north pole) & 132 & Kmag & mag & ? Ks magnitude \\
\hline 73 & e_Wvel & $\mathrm{km} \mathrm{s}^{-1}$ & ? uncertainty on Wvel & 133 & e_Kmag & mag & ? uncertainty on Kmag \\
\hline 74 & Xpos & $\mathrm{pc}$ & ? Galactic position $X$ (toward & 134 & q_Kmag & $\cdots$ & quality of Kmag \\
\hline & & & Galactic center) & 135 & refJHK & $\cdots$ & Reference for Infrared \\
\hline 75 & e_Xpos & $\mathrm{pc}$ & ? uncertainty on Xpos & & & & photometry \\
\hline 76 & Ypos & $\mathrm{pc}$ & $\begin{array}{l}\text { ? Galactic position } Y \text { direction } \\
\text { (toward solar motion) }\end{array}$ & 136 & jointJHK & $\cdots$ & $\begin{array}{l}\text { [JD] Infrared joint photometry } \\
\text { flag (4) }\end{array}$ \\
\hline 77 & e_Ypos & $\mathrm{pc}$ & ? uncertainty on Ypos & 137 & W1mag & mag & ? W1 magnitude \\
\hline 78 & Zpos & $\mathrm{pc}$ & ? Galactic position $Z$ direction & 138 & e_W1mag & mag & ? uncertainty on W1mag \\
\hline & & & (toward Galactic north pole) & 139 & q_W1mag & $\cdots$ & quality of W1mag \\
\hline 79 & e_Zpos & $\mathrm{pc}$ & ? uncertainty on Zpos & 140 & W2mag & mag & ? W2 magnitude \\
\hline 80 & refUVWXYZ & $\cdots$ & Reference for Galactic velocity & 141 & e_W2mag & mag & ? uncertainty on W2mag \\
\hline & & & and position & 142 & q_W2mag & $\cdots$ & quality of W2mag \\
\hline 81 & FUV & mag & ? GALEX FUV magnitude & 143 & W3mag & mag & ? W3 magnitude \\
\hline 82 & e_FUV & mag & ? uncertainty on FUV & 144 & e_W3mag & mag & ? uncertainty on $\mathrm{W} 3 \mathrm{mag}$ \\
\hline 83 & NUV & mag & ? GALEX NUV magnitude & 145 & q_W3mag & $\cdots$ & quality of W3mag \\
\hline 84 & e_NUV & mag & ? uncertainty on NUV & 146 & W4mag & mag & ? W4 magnitude \\
\hline 85 & refUV & $\cdots$ & Reference for $G A L E X$ & 147 & e_W4mag & mag & ? uncertainty on W4mag \\
\hline & & & magnitude & 148 & q_W4mag & $\cdots$ & Quality of W4mag \\
\hline 86 & u'mag & mag & ? uncertainty on u'mag & 149 & refWISE & $\cdots$ & \\
\hline
\end{tabular}


Table 3

(Continued)

\begin{tabular}{|c|c|c|c|}
\hline Number & Label & Units & Description \\
\hline & & & $\begin{array}{l}\text { Reference for WISE } \\
\text { photometry }\end{array}$ \\
\hline 150 & jointWISE & $\cdots$ & $\begin{array}{l}\text { [J] WISE joint photometry } \\
\quad \text { flag (4) }\end{array}$ \\
\hline 151 & photvar & mag & ? photometric variability \\
\hline 152 & f_photvar & $\cdots$ & filter of photometric variability \\
\hline 153 & Xray-PosErr & $\operatorname{arcsec}$ & $\begin{array}{l}\text { ? Offset between J2000 E1991 } \\
\text { position and X-ray source }\end{array}$ \\
\hline 154 & Xents & $\mathrm{ct} \mathrm{s}^{-1}$ & ? ROSAT X-ray counts \\
\hline 155 & e_Xcnts & $\mathrm{ct} \mathrm{s}^{-1}$ & ? uncertainty on Xcnts \\
\hline 156 & HR1 & $\cdots$ & ? ROSAT HR1 hardness ratio \\
\hline 157 & e_HR1 & $\cdots$ & ? uncertainty on HR1 \\
\hline 158 & HR2 & $\cdots$ & ? ROSAT HR2 hardness ratio \\
\hline 159 & e_HR2 & $\cdots$ & ? uncertainty on HR2 \\
\hline 160 & r_Xcnts & $\cdots$ & $\begin{array}{l}\text { Reference for ROSAT X-ray } \\
\text { detection }\end{array}$ \\
\hline 161 & n_Xcnts & $\cdots$ & $\begin{array}{l}\text { [JD] ROSAT joint photometry } \\
\quad \text { flag (4) }\end{array}$ \\
\hline 162 & VMag & mag & $\begin{array}{l}\text { ? Absolute } V \text { magnitude } \\
\quad \text { (calculated) }\end{array}$ \\
\hline 163 & B-V & mag & $? B-V$ color (calculated) \\
\hline 164 & V-K & mag & $? V-K s$ color (calculated) \\
\hline 165 & V-I & mag & $? V-I$ color $($ calculated $)$ \\
\hline 166 & $\mathrm{~J}-\mathrm{K}$ & mag & $? J-K s$ color (calculated) \\
\hline 167 & EB-V & mag & ? Reddening \\
\hline 168 & r_EB-V & $\cdots$ & reference for EB-V \\
\hline 169 & BolMag & mag & ? Bolometric magnitude \\
\hline 170 & e_BolMag & mag & ? uncertainty on BolMag \\
\hline 171 & r_BolMag & $\cdots$ & reference for BolMag \\
\hline 172 & $\mathrm{Lx}$ & {$[\mathrm{J}-7 / \mathrm{s}]$} & ? Log of X-ray flux \\
\hline 173 & e_Lx & {$[\mathrm{J}-7 / \mathrm{s}]$} & ? uncertainty on $\mathrm{Lx}$ \\
\hline 174 & LxLbol & $\ldots$ & $? \log (\mathrm{Lx} / \mathrm{Lbol})$ \\
\hline 175 & f_LxLbol & $\cdots$ & limit flag on LxLbol \\
\hline 176 & SpType & $\cdots$ & Spectral Type \\
\hline 177 & r_SpType & $\cdots$ & Reference for SpType \\
\hline 178 & n_SpType & $\cdots$ & $\begin{array}{l}\text { [JD] Spectral Type joint } \\
\text { flag (4) }\end{array}$ \\
\hline 179 & Teff1 & $\mathrm{K}$ & ? Effective Temperature 1 \\
\hline 180 & e_Teff1 & $\mathrm{K}$ & ? uncertainty on Teff 1 \\
\hline 181 & r_Teffl & $\cdots$ & reference for Teff1 \\
\hline 182 & Teff 2 & $\mathrm{~K}$ & ? Effective Temperature 2 \\
\hline 183 & e_Teff2 & $\mathrm{K}$ & $?$ uncertainty on Teff 2 \\
\hline 184 & r_Teff 2 & $\cdots$ & reference for Teff 2 \\
\hline 185 & Teff3 & $\mathrm{K}$ & ? Effective Temperature 3 \\
\hline 186 & e_Teff3 & $\mathrm{K}$ & ? uncertainty on Teff 3 \\
\hline 187 & r_Teff3 & $\cdots$ & reference for Teff3 \\
\hline 188 & Teff4 & $\mathrm{K}$ & ? Effective Temperature 4 \\
\hline 189 & e_Teff4 & $\mathrm{K}$ & ? uncertainty on Teff 4 \\
\hline 190 & r_Teff4 & $\cdots$ & reference for Teff 4 \\
\hline 191 & vsinil & $\mathrm{km} \mathrm{s}^{-1}$ & ? Rotational Velocity 1 \\
\hline 192 & e_vsinil & $\mathrm{km} \mathrm{s}^{-1}$ & ? uncertainty on vsinil \\
\hline 193 & f_vsinil & $\cdots$ & [ule] limit flag on vsini1 (5) \\
\hline 194 & r_vsinil & $\cdots$ & reference for vsinil \\
\hline 195 & vsini2 & $\mathrm{km} \mathrm{s}^{-1}$ & ? Rotational Velocity 2 \\
\hline 196 & e_vsini2 & $\mathrm{km} \mathrm{s}^{-1}$ & ? uncertainty on vsini 2 \\
\hline 197 & f_vsini2 & $\ldots$ & [ule] limit flag on vsini2 (5) \\
\hline 198 & r_vsini2 & $\cdots$ & reference for vsini 2 \\
\hline 199 & vsini3 & $\mathrm{km} \mathrm{s}^{-1}$ & ? Rotational Velocity 3 \\
\hline 200 & e_vsini3 & $\mathrm{km} \mathrm{s}^{-1}$ & ? uncertainty on vsini3 \\
\hline 201 & f_vsini3 & $\cdots$ & [ule] limit flag on vsini3 (5) \\
\hline 202 & r_vsini3 & $\mathrm{km} \mathrm{s}^{-1}$ & reference for vsini 3 \\
\hline 203 & CaHIndex & $\cdots$ & $\begin{array}{l}\text { ? } \mathrm{CaH} 697.5 \mathrm{~nm} 3 \mathrm{~nm} \text { absorp- } \\
\text { tion band Index }\end{array}$ \\
\hline 204 & e_CaHIndex & $\cdots$ & ? uncertainty on CaHIndex \\
\hline 205 & r_CaHIndex & $\cdots$ & reference for CaHIndex \\
\hline 206 & CaHNarrowIndex & $\cdots$ & $\begin{array}{l}\text { ? } \mathrm{CaH} 697.5 \mathrm{~nm} .5 \mathrm{~nm} \\
\text { absorption band Index }\end{array}$ \\
\hline
\end{tabular}

Table 3

(Continued)

\begin{tabular}{|c|c|c|c|}
\hline Number & Label & Units & Description \\
\hline 207 & e_CaHNarrowIndex & $\cdots$ & $\begin{array}{l}\text { ? uncertainty on } \\
\text { CaHNarrowIndex }\end{array}$ \\
\hline 208 & r_CaHNarrowIndex & $\cdots$ & reference for CaHNarrowIndex \\
\hline 209 & EWHal & $0.1 \mathrm{~nm}$ & $\begin{array}{l}\text { ? H-alpha }(656.3 \mathrm{~nm}) \text { equiva- } \\
\text { lent width } 1\end{array}$ \\
\hline 210 & e_EWHa1 & $0.1 \mathrm{~nm}$ & ? uncertainty on EWHal \\
\hline 211 & r_EWHal & $\cdots$ & reference for EWHal \\
\hline 212 & EWHa2 & $0.1 \mathrm{~nm}$ & $\begin{array}{l}\text { ? H-alpha }(656.3 \mathrm{~nm}) \text { equiva- } \\
\text { lent width } 2\end{array}$ \\
\hline 213 & e_EWHa2 & $0.1 \mathrm{~nm}$ & ? uncertainty on EWHa2 \\
\hline 214 & r_EWHa2 & $\ldots$ & reference for EWHa2 \\
\hline 215 & EWHa3 & $0.1 \mathrm{~nm}$ & $\begin{array}{l}\text { ? H-alpha }(656.3 \mathrm{~nm}) \text { equiva- } \\
\text { lent width } 3\end{array}$ \\
\hline 216 & r_EWHa3 & $\cdots$ & reference for EWHa3 \\
\hline 217 & EWLi1 & $0.1 \mathrm{pm}$ & $\begin{array}{l}\text { ? Lithium } 670.8 \mathrm{~nm} \text { doublet } \\
\text { equivalent width } 1\end{array}$ \\
\hline 218 & e_EWLi1 & $0.1 \mathrm{pm}$ & ? uncertainty on EWLil \\
\hline 219 & f_EWLi1 & $\ldots$ & [ule] limit flag on EWLi1 (5) \\
\hline 220 & r_EWLi1 & $\ldots$ & reference for EWLi1 \\
\hline 221 & EWLi2 & $0.1 \mathrm{pm}$ & $\begin{array}{l}\text { ? Lithium } 670.8 \mathrm{~nm} \text { doublet } \\
\text { equivalent width } 2\end{array}$ \\
\hline 222 & e_EWLi2 & $0.1 \mathrm{pm}$ & ? uncertainty on EWLi2 \\
\hline 223 & f_EWLi2 & $\ldots$ & [ule] limit flag on EWLi2 (5) \\
\hline 224 & r_EWLi2 & $\cdots$ & reference for EWLi2 \\
\hline 225 & EWLi3 & $0.1 \mathrm{pm}$ & $\begin{array}{l}\text { ? Lithium } 670.8 \mathrm{~nm} \text { doublet } \\
\text { equivalent width } 3\end{array}$ \\
\hline 226 & e_EWLi3 & $0.1 \mathrm{pm}$ & ? uncertainty on EWLi3 \\
\hline 227 & f_EWLi3 & $\cdots$ & [ule] limit flag on EWLi3 (5) \\
\hline 228 & r_EWLi3 & $\cdots$ & reference for EWLi3 \\
\hline 229 & EWLi4 & $0.1 \mathrm{pm}$ & $\begin{array}{l}\text { ? Lithium } 670.8 \mathrm{~nm} \text { doublet } \\
\text { equivalent width } 4\end{array}$ \\
\hline 230 & e_EWLi4 & $0.1 \mathrm{pm}$ & ? uncertainty on EWLi4 \\
\hline 231 & r_EWLi4 & $\cdots$ & reference for EWLi4 \\
\hline 232 & ALi1 & $\cdots$ & ? Lithium abundance 1 \\
\hline 233 & r_ALi1 & $\cdots$ & reference for ALi1 \\
\hline 234 & ALi2 & $\cdots$ & ? Lithium abundance 2 \\
\hline 235 & r_ALi2 & $\cdots$ & reference for $\mathrm{ALi} 2$ \\
\hline 236 & EWK7699 & $0.1 \mathrm{~nm}$ & $\begin{array}{l}\text { ? Potassium } 769.9 \mathrm{~nm} \text { equiva- } \\
\text { lent width }\end{array}$ \\
\hline 237 & e_EWK7699 & $0.1 \mathrm{~nm}$ & ? uncertainty on EWK7699 \\
\hline 238 & r_EWK7699 & $\cdots$ & reference for EW7699 \\
\hline 239 & EWNa8200 & $0.1 \mathrm{~nm}$ & $\begin{array}{l}\text { ? Sodium } 820.0 \mathrm{~nm} \text { equivalent } \\
\text { width }\end{array}$ \\
\hline 240 & e_EWNa8200 & $0.1 \mathrm{~nm}$ & ? uncertainty on EWNa8200 \\
\hline 241 & Na8200Index & $\cdots$ & $\begin{array}{l}\text { ? Sodium } 820.0 \mathrm{~nm} \text { gravity } \\
\text { index }\end{array}$ \\
\hline 242 & e_Na8200Index & $\cdots$ & ? uncertainty on Na8200Index \\
\hline 243 & r_Na8200Index & $\cdots$ & reference on $\mathrm{Na} 8200$ Index \\
\hline 244 & $\mathrm{FeH1}$ & [Sun] & ? Metallicity 1 \\
\hline 245 & e_FeH1 & [Sun] & ? uncertainty on FeH1 \\
\hline 246 & r_FeH1 & $\cdots$ & reference for $\mathrm{FeH} 1$ \\
\hline 247 & $\mathrm{FeH} 2$ & [Sun] & ? Metallicity 2 \\
\hline 248 & e_FeH2 & [Sun] & ? uncertainty on $\mathrm{FeH} 2$ \\
\hline 249 & r_FeH2 & $\cdots$ & reference for $\mathrm{FeH} 2$ \\
\hline 250 & $\mathrm{FeH} 3$ & [Sun] & ? Metallicity 3 \\
\hline 251 & r_FeH3 & $\cdots$ & reference for $\mathrm{FeH} 3$ \\
\hline 252 & $\mathrm{BaH}$ & [Sun] & ? Barium abundance \\
\hline 253 & e_BaH & [Sun] & ? uncertainty on $\mathrm{BaH}$ \\
\hline 254 & r_BaH & $\cdots$ & reference for $\mathrm{BaH}$ \\
\hline 255 & RHK1 & $\cdots$ & $\begin{array}{l}\text { ? Mount Wilson Activity } \\
\text { Index } 1\end{array}$ \\
\hline 256 & r_RHK1 & $\cdots$ & reference for RHK1 \\
\hline 257 & RHK2 & $\cdots$ & $\begin{array}{l}\text { ? Mount Wilson Activity } \\
\text { Index } 2\end{array}$ \\
\hline 258 & r_RHK2 & $\cdots$ & reference for RHK2 \\
\hline 259 & $\operatorname{logg} 1$ & $\cdots$ & ? Surface Gravity 1 \\
\hline 260 & e_logg1 & $\cdots$ & ? uncertainty on $\log g 1$ \\
\hline
\end{tabular}


Table 3

(Continued)

\begin{tabular}{|c|c|c|c|}
\hline Number & Label & Units & Description \\
\hline 261 & $r_{-} \operatorname{logg} 1$ & $\cdots$ & reference for $\operatorname{logg} 1$ \\
\hline 262 & $\operatorname{logg} 2$ & $\cdots$ & ? Surface Gravity 2 \\
\hline 263 & e_logg2 & $\ldots$ & ? uncertainty on $\operatorname{logg} 2$ \\
\hline 264 & r_logg2 & $\ldots$ & reference for $\operatorname{logg} 2$ \\
\hline 265 & GROUP & $\ldots$ & Final Membership \\
\hline 266 & q_GROUP & $\cdots$ & quality of GROUP \\
\hline 267 & Age & Myr & ? Stellar Age \\
\hline 268 & r_Age & $\ldots$ & reference for Age \\
\hline 269 & GROUP1 & $\ldots$ & Membership 1 \\
\hline 270 & q_GROUP1 & $\cdots$ & quality of GROUP1 (6) \\
\hline 271 & r_GROUP1 & $\ldots$ & reference for GROUP1 \\
\hline 272 & GROUP2 & $\ldots$ & Membership 2 \\
\hline 273 & q_GROUP2 & $\ldots$ & quality of GROUP2 (6) \\
\hline 274 & r_GROUP2 & $\cdots$ & reference for GROUP2 \\
\hline 275 & GROUP3 & $\ldots$ & Membership 3 \\
\hline 276 & q_GROUP3 & $\cdots$ & quality of GROUP3 (6) \\
\hline 277 & r_GROUP3 & $\cdots$ & reference for GROUP3 \\
\hline 278 & GROUP4 & $\cdots$ & Membership 4 \\
\hline 279 & q_GROUP4 & $\cdots$ & quality of GROUP4 (6) \\
\hline 280 & r_GROUP4 & $\cdots$ & reference for GROUP4 \\
\hline 281 & GROUP5 & $\ldots$ & Membership 5 \\
\hline 282 & q_GROUP5 & $\ldots$ & quality of GROUP5 (6) \\
\hline 283 & r_GROUP5 & $\ldots$ & reference for GROUP5 \\
\hline 284 & GROUP6 & $\cdots$ & Membership 6 \\
\hline 285 & q_GROUP6 & $\ldots$ & quality of GROUP6 (6) \\
\hline 286 & r_GROUP6 & $\ldots$ & reference for GROUP6 \\
\hline 287 & GROUP7 & $\ldots$ & Membership 7 \\
\hline 288 & q_GROUP7 & $\cdots$ & quality of GROUP7 (6) \\
\hline 289 & r_GROUP7 & $\ldots$ & reference for GROUP7 \\
\hline 290 & GROUP8 & $\cdots$ & Membership 8 \\
\hline 291 & q_GROUP8 & $\cdots$ & quality of GROUP8 (6) \\
\hline 292 & r_GROUP8 & $\ldots$ & reference for GROUP8 \\
\hline 293 & GROUP9 & $\cdots$ & Membership 9 \\
\hline 294 & q_GROUP9 & $\cdots$ & quality of GROUP9 (6) \\
\hline 295 & r_GROUP9 & $\cdots$ & reference for GROUP9 \\
\hline 296 & GROUP10 & $\ldots$ & Membership 10 \\
\hline 297 & q_GROUP10 & $\ldots$ & quality of GROUP10 (6) \\
\hline 298 & r_GROUP10 & $\cdots$ & reference for GROUP10 \\
\hline 299 & GROUP11 & $\cdots$ & Membership 11 \\
\hline 300 & q_GROUP11 & $\cdots$ & quality of GROUP11 (6) \\
\hline 301 & r_GROUP11 & $\cdots$ & reference for GROUP11 \\
\hline 302 & LACEwING-F & $\cdots$ & $\begin{array}{l}\text { LACEwING Identification } \\
\text { (Field Star Mode) }\end{array}$ \\
\hline 303 & q_LACEwING-F & $\%$ & ? quality of LACEwING-F \\
\hline 304 & LACEwING-Y & $\cdots$ & $\begin{array}{l}\text { LACEwING Identification } \\
\text { (Young Star Mode) }\end{array}$ \\
\hline 305 & q_LACEwING-Y & $\%$ & ? quality of LACEwING-Y \\
\hline 306 & HRV1 & $\mathrm{km} \mathrm{s}^{-1}$ & ? Radial Velocity 1 (7) \\
\hline 307 & e_HRV1 & $\mathrm{km} \mathrm{s}^{-1}$ & ? uncertainty on HRV1 \\
\hline 308 & q_HRV1 & $\ldots$ & [e] quality of HRV1 \\
\hline 309 & r_HRV1 & $\cdots$ & reference for HRV1 \\
\hline 310 & HRV2 & $\mathrm{km} \mathrm{s}^{-1}$ & ? Radial Velocity 2 (7) \\
\hline 311 & e_HRV2 & $\mathrm{km} \mathrm{s}^{-1}$ & ? uncertainty on HRV2 \\
\hline 312 & q_HRV2 & $\cdots$ & [e] quality of HRV2 \\
\hline 313 & r_HRV2 & $\ldots$ & reference for HRV2 \\
\hline 314 & HRV3 & $\mathrm{km} \mathrm{s}^{-1}$ & ? Radial Velocity 3 (7) \\
\hline 315 & e_HRV3 & $\mathrm{km} \mathrm{s}^{-1}$ & ? uncertainty on HRV3 \\
\hline 316 & q_HRV3 & $\cdots$ & [e] quality of HRV3 \\
\hline 317 & r_HRV3 & $\ldots$ & reference for HRV3 \\
\hline 318 & HRV4 & $\mathrm{km} \mathrm{s}^{-1}$ & ? Radial Velocity 4 (7) \\
\hline 319 & e_HRV4 & $\mathrm{km} \mathrm{s}^{-1}$ & ? uncertainty on HRV4 \\
\hline 320 & q_HRV4 & $\ldots$ & [e] quality of HRV4 \\
\hline 321 & r_HRV4 & $\cdots$ & reference for HRV4 \\
\hline 322 & HRV5 & $\mathrm{km} \mathrm{s}^{-1}$ & ? Radial Velocity 5 (7) \\
\hline 323 & e_HRV5 & $\mathrm{km} \mathrm{s}^{-1}$ & ? uncertainty on HRV5 \\
\hline 324 & q_HRV5 & $\ldots$ & [e] quality of HRV5 \\
\hline
\end{tabular}

Table 3

(Continued)

\begin{tabular}{|c|c|c|c|}
\hline Number & Label & Units & Description \\
\hline 325 & r_HRV5 & $\cdots$ & reference for HRV5 \\
\hline 326 & HRV6 & $\mathrm{km} \mathrm{s}^{-1}$ & ? Radial Velocity 6 (7) \\
\hline 327 & e_HRV6 & $\mathrm{km} \mathrm{s}^{-1}$ & ? uncertainty on HRV6 \\
\hline 328 & q_HRV6 & $\cdots$ & [e] quality of HRV6 \\
\hline 329 & r_HRV6 & $\cdots$ & reference for HRV6 \\
\hline 330 & HRV7 & $\mathrm{km} \mathrm{s}^{-1}$ & ? Radial Velocity 7 (7) \\
\hline 331 & e_HRV7 & $\mathrm{km} \mathrm{s}^{-1}$ & ? uncertainty on HRV7 \\
\hline 332 & q_HRV7 & $\cdots$ & [e] quality of HRV7 \\
\hline 333 & r_HRV7 & $\ldots$ & reference for HRV7 \\
\hline 334 & HRV8 & $\mathrm{km} \mathrm{s}^{-1}$ & ? Radial Velocity 8 (7) \\
\hline 335 & e_HRV8 & $\mathrm{km} \mathrm{s}^{-1}$ & ? uncertainty on HRV8 \\
\hline 336 & r_HRV8 & $\cdots$ & reference for HRV8 \\
\hline 337 & plx1 & mas & ? Parallax 1 (7) \\
\hline 338 & e_plx1 & mas & ? uncertainty on plx 1 \\
\hline 339 & r_plx1 & $\cdots$ & reference for plx 1 \\
\hline 340 & plx2 & mas & ? Parallax 2 (7) \\
\hline 341 & e_plx2 & mas & ? uncertainty on plx 2 \\
\hline 342 & r_plx2 & $\cdots$ & reference for plx 2 \\
\hline 343 & plx3 & mas & ? Parallax 3 (7) \\
\hline 344 & e_plx3 & mas & ? uncertainty on plx 3 \\
\hline 345 & r_plx3 & $\cdots$ & reference for plx 3 \\
\hline 346 & plx4 & mas & ? Parallax 4 (7) \\
\hline 347 & e_plx4 & mas & ? uncertainty on plx 4 \\
\hline 348 & r_plx4 & $\cdots$ & reference for plx 4 \\
\hline 349 & plx5 & mas & ?Parallax 5 (7) \\
\hline 350 & e_plx5 & mas & ? uncertainty on plx 5 \\
\hline 351 & r_plx5 & $\cdots$ & reference for plx 5 \\
\hline 352 & plx6 & mas & ? Parallax 6 (7) \\
\hline 353 & e_plx6 & mas & ? uncertainty on plx6 \\
\hline 354 & r_plx6 & $\cdots$ & reference for plx6 \\
\hline 355 & plx7 & mas & ? Parallax 7 (7) \\
\hline 356 & e_plx7 & mas & ? uncertainty on plx 7 \\
\hline 357 & r_plx7 & $\cdots$ & reference for plx7 \\
\hline 358 & plx8 & mas & ? Parallax 8 (7) \\
\hline 359 & e_plx8 & mas & ? uncertainty on plx 8 \\
\hline 360 & r_plx8 & $\cdots$ & reference for plx 8 \\
\hline
\end{tabular}

Note. Guide to the Contents of the Catalog of Suspected Nearby Young Stars, which is available as a machine-readable table. Note that multiple column blocks are present for certain quantities $\left(T_{\text {eff }}, \mathrm{EWHa}, \mathrm{EWLi}\right.$, etc.) in the interest of preserving every published data value. These should not inherently be preferred over each other, except that the weighted mean parallax columns (\#47-\#48) and weighted mean RV columns (\#49-\#50) should be preferred over the individual measurements that were combined to produce them (\#306\#360). (1) LiSample flags are as follows. $\mathrm{L}=$ star has a measured lithium EW greater than $10 \AA$ and $2 \sigma$ of the published error or $50 \mathrm{~m} \AA$. $\mathrm{F}=$ star has lithium, but is a member of a more distant group beyond $120 \mathrm{pc} . \mathrm{A}=\mathrm{star}$ is in the same star system as a lithium-sample star. Lower-case letters $1, \mathrm{f}$, a indicate the objects are not system primaries. (2) Bona fide flags are $\mathrm{B}=$ in final bona fide sample; $\mathrm{R}=$ rejected from initial high-confidence sample; lower case letters $b, r$ indicate the objects are not system primaries. (3) Multiplicity types are $\mathrm{AB}$ for astrometric binaries, $\mathrm{IB}$ for interferometric binaries, $\mathrm{EB}$ for eclipsing binaries, $\mathrm{OB}$ for occultation binaries, $\mathrm{VB}$ for visual binaries; $\mathrm{SB}$ for spectroscopic binaries; S for spectroscopic single stars; SB1, SB2, SB3 for single-, double-, and triple-lined spectroscopic binaries. (4) Joint flags: $\mathbf{J}=$ quoted quantity actually refers to an unresolved detection that includes this object. $\mathrm{D}=$ quoted quantity has been deblended into approximate individual measurements. (5) Limit flags: $1=$ lower limit, $\mathrm{u}=$ upper limit, $\mathrm{e}=$ uncertainty set to typical value. (6) Quality strings have been reproduced from the source papers and are a mix of different scales, with the exception of "BF," which always denotes an identification as a bona fide member. (7) Source RVs and parallaxes for the weighted means in columns 47-50.

(This table is available in its entirety in machine-readable form.) 
et al. (2010), Murphy (2012), Gagné et al. (2014b), Schneider et al. (2013, 2014), and Riedel et al. (2014).

3 . Young objects reported in papers that did not consider group memberships, as reported by Guillout et al. (2009), Mishenina et al. (2012), and Xing \& Xing (2012).

4. Field (and variants like "young disk") objects considered in papers on young stars and never reported as young, particularly from Montes et al. (2001b), Makarov (2007), Shkolnik et al. (2009, 2011), López-Santiago et al. (2010b), Maldonado et al. (2010), Rodriguez et al. (2011), McCarthy \& Wilhelm (2014), Gagné et al. (2014b), Riedel et al. (2014), and Klutsch et al. (2014).

All data relevant to population studies of stellar youth and membership have been taken from these source papers. Care has been taken to homogenize the data as much as possible: upper limit flags have been added, $\mathrm{H} \alpha \mathrm{EW}$ has been standardized as negative when in emission, and lithium EW is uniformly recorded in milliangstroms.

\subsection{Survey Sources}

The catalog is supplemented by additional data sources drawn from large surveys. This aids in providing useful information about the stars and strengthens the consistency of the source data.

\subsubsection{Astrometry}

Positions and proper motions were preferentially sourced from the following ICRS catalogs tied to the Hipparcos reference frame:

1. Hipparcos 2 (van Leeuwen 2007), 1915 objects, 0.1-3.0

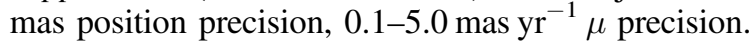

2. UCAC4 (Zacharias et al. 2013), 1527 objects, 10-100

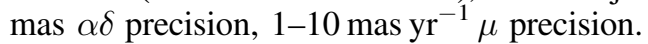

3. Tycho-2 (Høg et al. 2000), 906 objects, 10-100 mas $\alpha \delta$

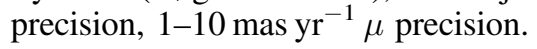

4. PPMXL (Röser et al. 2010), 712 objects, 50-100 mas $\alpha \delta$

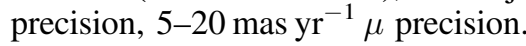

5. 2MASS and 2MASS-6X (Skrutskie et al. 2006). 286 objects, 60-120 mas $\alpha \delta$ precision, no $\mu$.

6. SDSS DR9 (Ahn et al. 2012) 4 objects, 80-200 mas $\alpha \delta$ precision, no $\mu$.

7. Source papers (from Table 4). These mostly provide

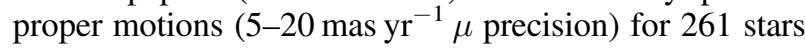
found in 2MASS or 2MASS-6X. The other 23 have proper motions copied from a primary star.

There are still 43 objects (35 of which are in the Pleiades) that have no reported proper motions. Parallaxes were sourced from papers listed in Table 5. Where available, parallaxes for objects in multiple systems and multiple observations of the same stars were combined into weighted mean system parallaxes, under the assumptions that the published uncertainties are accurate and that the parallax of every component of the system is the same to within measurement errors.

\subsubsection{Radial Velocity}

Radial velocities were assumed to apply to all companions within roughly $2^{\prime \prime}$. Given that it is both possible and likely that different members of a multiple star system have different radial velocities, when different components have independently measured $\gamma$, they were combined using a weighted standard deviation to produce a systemic velocity.

Attempts have been made to reduce the double-counting of $\gamma$, particularly in cases where a later paper cited a $\gamma$ taken from one of the catalogs included here. This has particularly been a problem for Barbier-Brossat \& Figon (2000) and Kharchenko et al. (2007), which both contain the General Catalog of Radial Velocities (Wilson 1953) where uncertainties were reported as letter codes. Barbier-Brossat \& Figon (2000) and Kharchenko et al. (2007) recommend a translation of the letter code into $\mathrm{km} \mathrm{s}^{-1}$ uncertainty different from the VizieR version of Wilson (1953; and papers that cite it directly, such as Malo et al. 2013), which it does, leading to nearly identical $\gamma$ appearing in different sources.

In many cases, $\gamma$ have been published without uncertainties. Because our weighted standard deviations require an uncertainty, we have invented them where necessary, and flagged them with "e" in our source tables. Radial velocities with quality codes, originating from Wilson (1953), had uncertainties assigned according to the quality notes as suggested in the table notes; where no quality code was available, we have set the errors to $3.7 \mathrm{~km} \mathrm{~s}^{-1}$, equivalent to letter code "C." Most other papers were given $1 \mathrm{~km} \mathrm{~s}^{-1}$ uncertainties, as per Table 6 .

\subsubsection{Photometry}

Photometry came from numerous sources and was applied in a set order, presented here in decreasing order of preference. For optical data:

1. Photometry from source papers (except van Altena et al. 1995).

2. Southern Proper Motion (SPM4) catalog, CCD second epoch measurements only (Girard et al. 2011)-B, $V$ only.

3. The American Association of Variable Star Observers Photometric All Sky Survey (APASS DR9, Henden et al. 2016), where all stars have been proper-motioncorrected to epoch 2011 January 1, roughly the midpoint of the survey. $B, V$ only.

4. APASS DR6 data, as incorporated into the Fourth United States Naval Observatory Compiled Astrometric Catalog (UCAC4; Zacharias et al. 2013)—B, $V$ only.

The APASS DR9 data did not completely replace UCAC4's APASS DR6 data due to better position solution and crossmatching done when UCAC4 incorporated APASS DR6.

SDSS $u^{\prime} g^{\prime} r^{\prime} i^{\prime} z^{\prime}$ photometry was sourced from

1. The Sloan Digital Sky Survey (SDSS9; Ahn et al. 2012).

2. APASS DR9 (Henden et al. 2016), corrected for proper motion to 2011 January 1 ( $g^{\prime} r^{\prime} i^{\prime}$ only).

3. UCAC4 (Zacharias et al. 2013) ( $g^{\prime} r^{\prime} i^{\prime}$ only).

Near-infrared data were sourced from our source papers, if they deblended photometry (only Riedel et al. 2014), or from the Two Micron All-Sky Survey (Skrutskie et al. 2006). Midinfrared data in the WISE $W 1, W 2, W 3$, and $W 4$ photometric bands were sourced from the ALLWISE catalog (Cutri et al. 2013), which supersedes WISE All-Sky data (Cutri et al. 2012).

X-ray data were extracted from the ROSAT All-Sky Survey's bright star catalog (Voges et al. 1999) and faint star catalog (Voges et al. 2000) using an aperture of 25" around all targets, after they were corrected by proper motion to their 1991 
Table 4

Source Papers

\begin{tabular}{|c|c|}
\hline Citation & Groups Included $^{\mathrm{a}}$ \\
\hline Eggen (1991) & IC 2391 Supercluster \\
\hline Barrado y Navascues (1998) & Castor \\
\hline Makarov \& Urban (2000) & Car-Vela \\
\hline van den Ancker et al. (2000) & Capricornus \\
\hline Montes et al. (2001b) & Multiple \\
\hline Montes et al. (2001a) & Multiple \\
\hline King et al. (2003) & Ursa Major \\
\hline Ribas (2003) & Castor \\
\hline Zuckerman \& Song (2004) & Multiple \\
\hline Mamajek (2005) & TW Hya \\
\hline Casewell et al. (2006) & Coma Ber \\
\hline López-Santiago et al. (2006) & Multiple \\
\hline Moór et al. (2006) & Multiple \\
\hline Zuckerman et al. (2006) & Car-Near \\
\hline Guenther et al. (2007) & Multiple \\
\hline Kraus \& Hillenbrand (2007) & Coma Ber \\
\hline Makarov (2007) & Multiple \\
\hline Platais et al. (2007) & IC 2391 Cluster \\
\hline Stauffer et al. (2007) & Pleiades \\
\hline Kirkpatrick et al. (2008) & TW Hya \\
\hline Mentuch et al. (2008) & Multiple \\
\hline Teixeira et al. (2008) & TW Hya \\
\hline Torres et al. (2008, p. 757) & Multiple \\
\hline da Silva et al. (2009) & Multiple \\
\hline Guillout et al. (2009) & Other \\
\hline Lépine \& Simon (2009) & $\beta$ Pic \\
\hline Shkolnik et al. (2009) & Other \\
\hline Teixeira et al. (2009) & $\beta$ Pic, TW Нyа \\
\hline Caballero (2010) & Castor \\
\hline López-Santiago et al. (2010b) & Multiple \\
\hline López-Santiago et al. (2010a) & $\eta$ Cha \\
\hline Maldonado et al. (2010) & Multiple \\
\hline Murphy et al. (2010) & $\eta$ Cha \\
\hline Nakajima et al. (2010) & Multiple \\
\hline Rice et al. (2010) & $\beta$ Pic \\
\hline Schlieder et al. (2010) & $\beta$ Pic, AB Dor \\
\hline Yee \& Jensen (2010) & $\beta$ Pic \\
\hline Kiss et al. (2011) & Multiple \\
\hline Riedel et al. (2011) & Argus \\
\hline Rodriguez et al. (2011) & TW Hya, Sco-Cen \\
\hline Shkolnik et al. (2011) & TW Hya \\
\hline Wahhaj et al. (2011) & AB Dor \\
\hline Zuckerman et al. (2011) & Multiple \\
\hline Kastner et al. (2012) & $\epsilon$ Cha \\
\hline McCarthy \& White (2012) & $\beta$ Pic, AB Dor \\
\hline Mishenina et al. (2012) & Other \\
\hline Murphy (2012) & Other \\
\hline Nakajima \& Morino (2012) & Multiple \\
\hline Schlieder et al. (2012b) & $\beta$ Pic, AB Dor \\
\hline Schlieder et al. (2012c) & $\beta$ Pic, AB Dor \\
\hline Shkolnik et al. (2012) & Multiple \\
\hline Schneider et al. (2012a) & TW Hya \\
\hline Schneider et al. (2012b) & TW Hya \\
\hline Xing \& Xing (2012) & Other \\
\hline Barenfeld et al. (2013) & AB Dor \\
\hline Delorme et al. (2013) & Tuc-Hor \\
\hline De Silva et al. (2013) & Argus \\
\hline Eisenbeiss et al. (2013) & Her-Lyr \\
\hline Hinkley et al. (2013) & Other \\
\hline Liu et al. (2013b) & $\beta \mathrm{Pic}$ \\
\hline Malo et al. (2013) & Multiple \\
\hline Mamajek et al. (2013) & Other \\
\hline Moór et al. (2013) & Multiple \\
\hline Murphy et al. (2013) & $\eta$ Cha, $\epsilon$ Cha \\
\hline
\end{tabular}

Table 4

(Continued)

\begin{tabular}{ll}
\hline \hline Citation & Groups Included $^{\mathrm{a}}$ \\
\hline Rodriguez et al. (2013) & Multiple \\
Schneider et al. (2013) & Other \\
Zuckerman et al. (2013) & Oct-Near \\
Weinberger et al. (2013) & TW Hya \\
Binks \& Jeffries (2014) & $\beta$ Pic \\
Casewell et al. (2014) & Coma Ber, Hyades \\
Klutsch et al. (2014) & Multiple \\
Kraus et al. (2014a) & Tuc-Hor \\
Gagné et al. (2014b) & Multiple \\
Gagné et al. (2014a) & TW Hya \\
Gagné et al. (2014c) & Argus \\
Malo et al. (2014a) & Multiple \\
Malo et al. (2014b) & Multiple \\
Mamajek \& Bell (2014) & $\beta$ Pic \\
McCarthy \& Wilhelm (2014) & AB Dor \\
Riedel et al. (2014) & Multiple \\
Rodriguez et al. (2014) & $\beta$ Pic \\
Schneider et al. (2014) & Other \\
Gagné et al. (2015) & Multiple \\
Murphy \& Lawson (2015) & Octans \\
E. E. Mamajek (2016, private communication) & Multiple
\end{tabular}

Notes. The papers that make up the membership of the catalog of young stars, along with the groups considered.

a Papers marked "Multiple" consider multiple groups; papers marked "Other" consider nearby young stars but do not identify them as members of any groups.

January 1 positions (the rough median date of the survey). Ultraviolet data from GALEX were extracted from the All-Sky Imaging Survey and Medium-Depth Imaging Survey (Bianchi et al. 2011) after correcting all stars to their 2007 January 1 positions.

Deblending magnitudes is possible (Riedel et al. 2014) but cannot be done systematically for all stars. The source of the majority of our multiplicity information, the Washington Double Star Catalog (WDS; Mason et al. 2015), does not report filters with its delta magnitudes on the most readily available public versions (VizieR, USNO text tables ${ }^{10}$ ).

\subsubsection{Multiplicity}

For the purposes of this paper, the multiplicity information in the catalog is not complete. The fundamental unit of the catalog is intended to be the single object, with one object per entry even if no information is known other than that the object exists.

The question of multiplicity for members of NYMGs is occasionally difficult, as some independent members of moving groups may be picked up by surveys as extremely wide common proper motion binaries (Caballero 2009, 2010; Shaya \& Olling 2011). Apart from well-known binaries, we have set an informal limit of $500^{\prime \prime}$ for binaries.

WDS contains information on multiples observed through direct imaging, adaptive optics, micrometery, and speckle interferometry, and is our primary source for multiplicity information. While there are catalogs of spectroscopic orbits (SB9; Pourbaix et al. 2004), there is no comparable central

\footnotetext{
${ }^{10}$ http://ad.usno.navy.mil/wds/, checked 2016 October 5.
} 
Table 5

Survey Papers

\begin{tabular}{|c|c|}
\hline Citation & Data Used \\
\hline Houk \& Cowley (1975) & Spectral Types \\
\hline Houk (1978) & Spectral Types \\
\hline Houk (1982) & Spectral Types \\
\hline Andersen et al. (1991) & Multiplicity \\
\hline Gliese \& Jahreiß (1991) & Spectral Types \\
\hline Hoffleit \& Jaschek (1991) & Catalog Names \\
\hline Kirkpatrick et al. (1991) & Spectral Types \\
\hline Cannon \& Pickering (1993) & Catalog Names \\
\hline Gatewood et al. (1993) & $\pi$ \\
\hline Gatewood (1995) & $\pi$ \\
\hline Gatewood \& de Jonge (1995) & $\pi$ \\
\hline Reid et al. (1995) & Spectral Types \\
\hline van Altena et al. (1995) (YPC4) & $\pi$, Opt phot. \\
\hline Covino et al. (1997) & $\gamma, \mathrm{Li}$ \\
\hline Benedict et al. (1999) & $\pi$ \\
\hline Söderhjelm (1999) & $\pi^{\mathrm{a}}$ \\
\hline Voges et al. (1999) (RASS-BSC) & X-ray phot. \\
\hline Weis et al. (1999) & $\pi$ \\
\hline Barbier-Brossat \& Figon (2000) (GCMRV) & $\gamma$ \\
\hline Benedict et al. (2000) & $\pi$ \\
\hline Ducati et al. (2001) & Opt phot. \\
\hline Høg et al. (2000) (TYCHO-2) & pos., $\mu$, Opt phot. \\
\hline Voges et al. (2000) (RASS-FSC) & $\mathrm{X}$-ray phot. \\
\hline Dahn et al. (2002) & $\pi$ \\
\hline Gizis et al. (2002) & $\gamma$ \\
\hline Henry et al. (2002) & Spectral Types \\
\hline Nidever et al. (2002) & $\gamma$ \\
\hline Torres \& Ribas (2002) & $\pi^{\mathrm{a}}$ \\
\hline Cutri et al. (2003) (2MASS) & pos., NIR phot. \\
\hline Song et al. (2003) & $\gamma$ \\
\hline Thorstensen \& Kirkpatrick (2003) & $\pi$ \\
\hline McArthur et al. (2004) & $\pi$ \\
\hline Pourbaix et al. (2004) (SB9) & Multiplicity \\
\hline Vrba et al. (2004) & $\pi$ \\
\hline Costa et al. (2005) & $\pi$ \\
\hline Jao et al. (2005) & $\pi$ \\
\hline Lépine \& Shara (2005) & $\mu$ \\
\hline Soderblom et al. (2005) & $\pi$ \\
\hline Valenti \& Fischer (2005) & $\gamma$, vsini \\
\hline Benedict et al. (2006) & $\pi$ \\
\hline Gontcharov (2006) & $\gamma$ \\
\hline Gray et al. (2006) & Spectral Types \\
\hline Henry et al. (2006) & $\pi$, Opt phot. \\
\hline Torres et al. (2006) & $\gamma, \mathrm{H} \alpha, \mathrm{Li}, \mathrm{vsini}$ \\
\hline Biller \& Close (2007) & $\pi$ \\
\hline Close et al. (2007) & Spectral Types \\
\hline Daemgen et al. (2007) & Multiplicity \\
\hline Gizis et al. (2007) & $\pi$, phot. \\
\hline Kharchenko et al. (2007) & $\gamma$ \\
\hline Scholz et al. (2007) & $\gamma$ \\
\hline van Leeuwen (2007) (Hipparcos-2) & $\pi$, pos., $\mu$ \\
\hline Ducourant et al. (2008) & $\pi$ \\
\hline Fernández et al. (2008) & $\gamma$ \\
\hline Jameson et al. (2008) & $\mu$ \\
\hline Gatewood \& Coban (2009) & $\pi$ \\
\hline Subasavage et al. (2009) & $\pi$ \\
\hline Teixeira et al. (2009) & $\pi$ \\
\hline Bergfors et al. (2010) & Multiplicity \\
\hline Blake et al. (2010) & $\gamma$ \\
\hline Raghavan et al. (2010) & Multiplicity \\
\hline Riedel et al. (2010) & $\pi$, Opt phot. \\
\hline Röser et al. (2010) (PPMXL) & position, $\mu$, NIR phot. \\
\hline Shkolnik et al. (2010) & Multiplicity, $\gamma$ \\
\hline Smart et al. (2010) & $\pi$ \\
\hline
\end{tabular}

Table 5

(Continued)

\begin{tabular}{ll}
\hline \hline Citation & Data Used \\
\hline Stauffer et al. (2010) & Catalog Names \\
Bianchi et al. (2011) (GALEX DR5) & UV phot. \\
Girard et al. (2011) (SPM4) & Opt phot. \\
Messina et al. (2011) & Spectral Types \\
Moór et al. (2011) & $\gamma$ \\
von Braun et al. (2011) & $\pi$ \\
Ahn et al. (2012) (SDSS DR9) & pos., SDSS phot. \\
Allen et al. (2012) & $\mu$ \\
Bailey et al. (2012) & $\gamma$ \\
Bowler et al. (2012a) & Multiplicity \\
Bowler et al. (2012b) & Multiplicity \\
Dupuy \& Liu (2012) & $\pi$ \\
Faherty et al. (2012) & $\pi$ \\
Janson et al. (2012) & Multiplicity \\
Zacharias et al. (2013) (UCAC4) & pos., $\mu$, Opt, SDSS, NIR phot. \\
Bowler et al. (2013) & Multiplicity \\
Cutri et al. (2013) (ALLWISE) & MIR phot. \\
Kordopatis et al. (2013) (RAVE DR4) & $\gamma$ \\
Liu et al. (2013a) & $\pi$ \\
Marocco et al. (2013) & $\pi$ \\
Dieterich et al. (2014) & $\pi$, Opt phot. \\
Dittmann et al. (2014) & $\pi$ \\
Ducourant et al. (2014) & $\mu, \pi$ \\
Lurie et al. (2014) & $\pi$, Opt phot. \\
Naud et al. (2014) & Multiplicity \\
Zapatero Osorio et al. (2014) & $\pi$ \\
Elliott et al. (2015) & $\gamma$ \\
Henden et al. (2015) (APASS DR9) & Opt, SDSS phot. \\
Mason et al. (2015) (WDS) & Multiplicity \\
Faherty et al. (2016) & $\gamma$ \\
\hline & \\
\hline
\end{tabular}

Notes. Additional data sources used in the catalog.

${ }^{a}$ Parallax replaces Hipparcos data.

Table 6

Radial Velocity Uncertainty Defaults

\begin{tabular}{|c|c|c|}
\hline Citation & Uncertainty & Rationale \\
\hline Eggen (1991) & 5 & Comparison to other extant $\gamma$ \\
\hline $\begin{array}{l}\text { Barbier-Brossat \& } \\
\text { Figon (2000) }\end{array}$ & 3.7 & $\begin{array}{l}\text { Letter code } C \text {, unless a code was } \\
\text { given }\end{array}$ \\
\hline Montes et al. (2001b) & 1 & Typical uncertainty in paper \\
\hline Montes et al. (2001a) & 1 & Typical uncertainty in paper \\
\hline Gontcharov (2006) & 1 & Typical uncertainty in catalog \\
\hline Torres et al. (2006) & 1 & $\begin{array}{l}\text { Cited agreement with Nordström } \\
\text { et al. (2004) }\end{array}$ \\
\hline Kharchenko et al. (2007) & 3.7 & $\begin{array}{l}\text { Letter code } C \text { unless a code was } \\
\text { given }\end{array}$ \\
\hline Guillout et al. (2009) & 1 & Typical uncertainty in paper \\
\hline Maldonado et al. (2010) & 1 & Typical uncertainty in paper \\
\hline Murphy et al. (2010) & 2 & Typical uncertainty in paper \\
\hline Schlieder et al. (2010) & 2 & Typical uncertainty in paper \\
\hline Schneider et al. (2012a) & 2 & Typical uncertainty in paper \\
\hline De Silva et al. (2013) & 1 & Subsequent to Torres et al. (2006) \\
\hline Malo et al. (2013) & 1 & Typical uncertainty in paper \\
\hline Malo et al. (2014a) & 1 & Typical uncertainty in paper \\
\hline Malo et al. (2014b) & 1 & Typical uncertainty in paper \\
\hline Elliott et al. (2015) & 1 & Subsequent to Torres et al. (2006) \\
\hline
\end{tabular}


source for general spectroscopic binaries. Thus, all information on other, closer multiples has come from individual survey papers and system notes in WDS.

Companions listed in WDS have been accepted if and only if they have been observed more than once and are still consistent with being common proper motion pairs. Discovery papers generally contain the most reliable information about spectroscopic and visual binaries when discovered, but many papers included here are compilations themselves or deal with systems known elsewhere.

\section{Input Membership Data}

\subsection{A New Bona Fide Sample}

LACEwING requires kinematic models of the NYMGs. These are $U V W$ and $X Y Z$ ellipsoids fit to genuine members of the groups. To create a list of bona fide members, we have pulled previously identified bona fide members from the Catalog of Suspected Nearby Young Stars (Section 4). We then filtered out probable interlopers from the samples using the TRACEwING code (Section 3). The resulting filtered samples of bona fide moving group members were then fit with ellipsoids.

\subsubsection{Initial Member Data}

The starting point for our membership list is 546 stars from published lists of high-confidence members, most notably the BANYAN series of papers (Malo et al. 2013, Gagné et al. 2014b, and subsequent papers).

1. BANYAN papers (Malo et al. 2013), with additions and subtractions from Gagné et al. (2014b, 2015) and Malo et al. (2014a, 2014b). These papers list bona fide members of TW Hya, $\beta$ Pic, Tuc-Hor, Columba, Carina, Argus, and AB Dor. Bona fide members are "all stars with a good measurement of trigonometric distance, proper motion, Galactic space velocity and other youth indicators such as $\mathrm{H} \alpha$ emission, X-ray emission, appropriate location in the Hertzprung-Russel diagram, and lithium absorption" (Malo et al. 2013, pp. 2); in practice the youth indicator for most targets is X-ray emission.

2. King et al. (2003) performed a thorough kinematic, activity, and isochronal analysis of the Ursa Major moving group, which concluded with a list of 60 nearly assured members, broken into a nucleus of 14 systems and 46 other members. We adopt the 14 nucleus members as bona fide members of Ursa Major.

3. Eisenbeiss et al. (2013) conducted an analysis of Her-Lyr using gyrochronology, isochrone fits, lithium abundances, and chromospheric activity, concluding with the identification of seven "canonical" members, which we adopt as bona fide members.

4. Murphy et al. (2010) analyzed the $\eta$ Cha open cluster and reconsidered membership for the cluster using proper motions, surface gravity measurements, activity, and lithium. We adopt their list of $\eta$ Cha members as bona fide members. Not all of them have trigonometric parallaxes.

5. Murphy et al. (2013) analyzed the $\epsilon$ Cha moving group using techniques similar to those used for $\eta$ Cha. We adopt their list of $\epsilon$ Cha members as bona fide members.

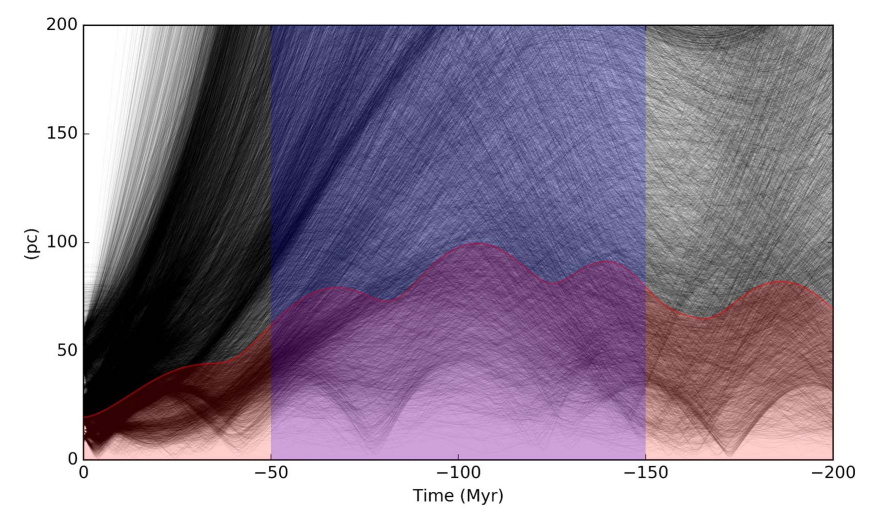

Figure 14. Epicyclic traceback of the entire AB Doradus moving group, showing the separations between each bona fide member (represented by 5000 random draws) and the center of the group, as a function of time.

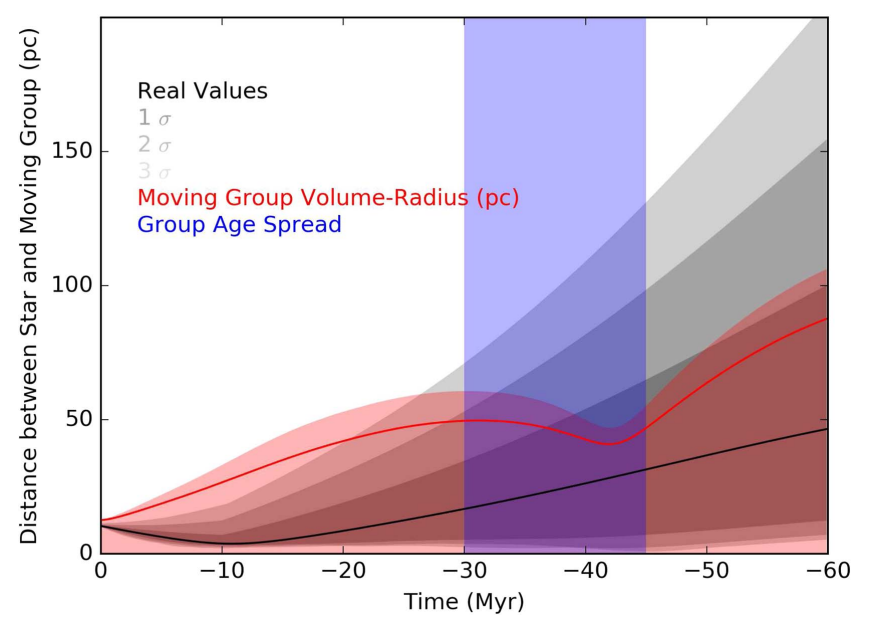

Figure 15. Same as Figure 7 except showing CPD-64 17 traced back relative to Tuc-Hor.

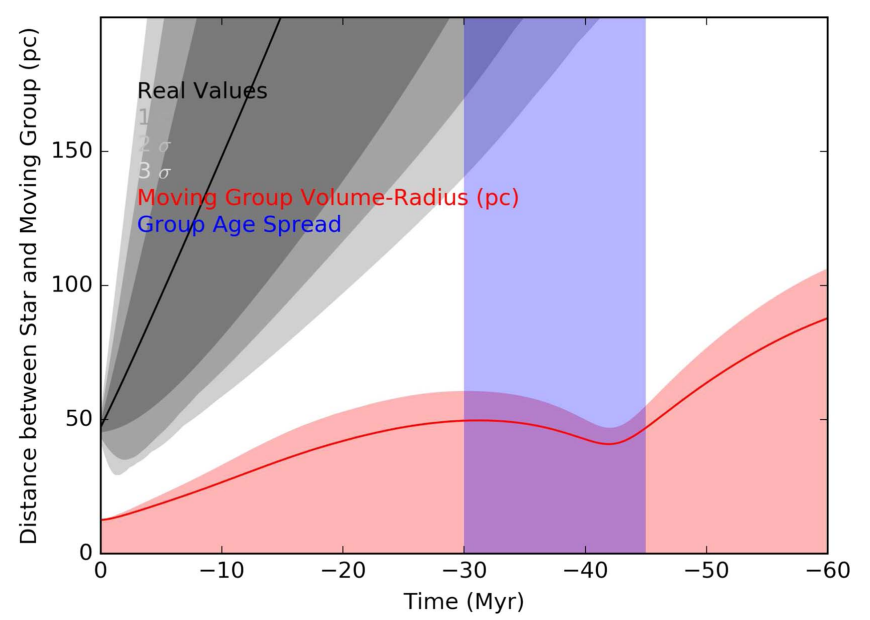

Figure 16. Same as Figure 7 except showing HIP 104308 traced back relative to Tuc-Hor.

Not all of these stars have measured trigonometric parallaxes either.

6. Murphy \& Lawson (2015) studied the Octans moving group using spectroscopy, photometry, and fast rotation. We adopt their list of Octans members as bona fide 

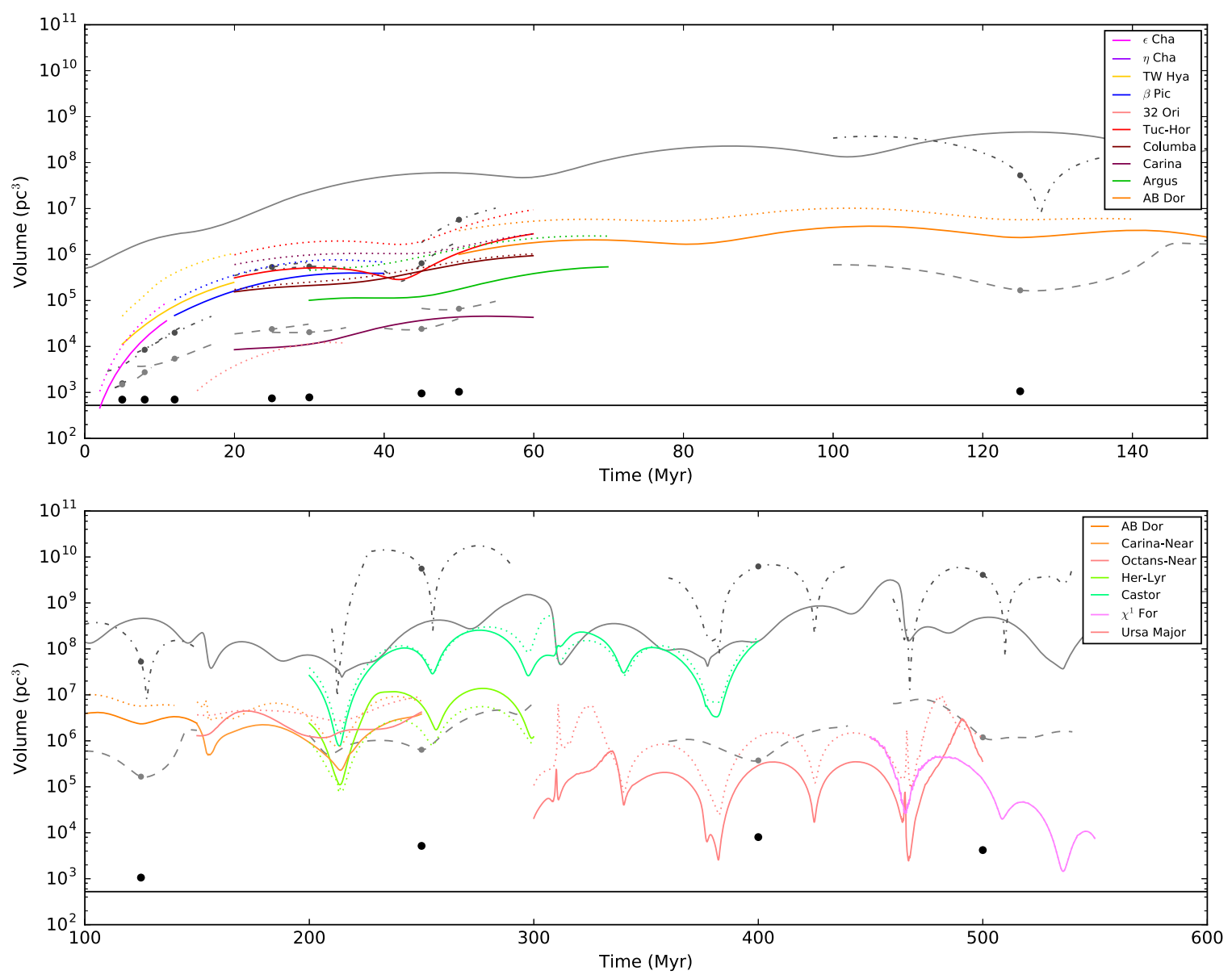

Figure 17. Volumes of the NYMGs around their supposed times of formation, before (dashed) and after (solid) outliers have been removed, plotted in Figure 10. All NYMGs are smaller than the simulated "real" moving group with current-precision kinematics (dashed-dotted curves); some are smaller than the "real" moving group with Gaia-precision kinematics (dashed curves); none are the size of the "real" moving group with no uncertainties (points). All, except Castor, are smaller than the "fake" moving group constructed from field stars (thick gray line).

members. None of them have measured trigonometric parallaxes.

7. Casewell et al. (2006) and Kraus \& Hillenbrand (2007) examined the Coma Ber open cluster for low-mass members, using proper motions and photometry for isochrone fits. We adopt their "known" members as bona fide members of Coma Ber.

8. Zuckerman et al. (2006) proposed a new moving group Car-Near. We take all reported members as bona fide members.

9. Zuckerman et al. (2013) proposed a new moving group, Oct-Near. We have taken all probable members as bona fide members.

10. E. E. Mamajek (2016, private communication) supplied a list of members of the 32 Ori and $\chi^{01}$ For moving groups (Mamajek 2015). We have taken all members rated as likely or definitive as bona fide members.

We have made several alterations to this list. PX Vir is listed by Eisenbeiss et al. (2013) as a canonical member of Her-Lyr and by both Malo et al. (2013) and Gagné et al. (2014b) as a bona fide member of $\mathrm{AB}$ Dor. It has been made a member of $\mathrm{AB}$ Dor (in the final analysis, it is a bona fide member of $\mathrm{AB}$ Dor and a bad match to Her-Lyr). Gagné et al. (2014b) has an erroneous entry for GJ 2079AB (HIP 50156) as a bona fide member of both $\beta$ Pic and Carina, when it is a modest probability member of
Carina (J. Gagné 2016, private communication) and was removed from the bona fide sample.

Several papers have removed targets from these lists. Hinkley et al. (2013), Barenfeld et al. (2013), and McCarthy \& Wilhelm (2014) have run more detailed analyses that have ruled out, or at least cast doubt on, members of Columba and AB Dor. The BANYAN papers (Gagné et al. 2014b; Malo et al. 2014a) have removed those and other targets from their bona fide lists, but we have retained all targets that were solely rejected for large uncertainties or discrepancies with their $X Y Z U V W$ model.

Another major difference with previous bona fide lists is that in the production of the catalog, we have reconsidered whether stars are parts of bound systems (AU Mic +AT Mic AB; $\beta^{01}$ Tuc $A B+\beta^{02}$ Tuc $A B+\beta^{03}$ Tuc AB; Mason et al. 2015). We only include the system primary in our kinematic analysis, and therefore have fewer systems in our initial and cleaned bona fide samples.

\subsubsection{Bona Fide Candidate Filtering}

The bona fide list of members was filtered using TRACEwING (Section 3) to identify and remove outliers: stars that could not possibly have been in the same location as the rest of the group at the time of formation. Moving groups were generated from the bona fide list (Figure 14), and then every member of 
Table 7

Moving Group Kinematic Properties

\begin{tabular}{|c|c|c|c|c|c|c|c|c|c|c|}
\hline \multirow[b]{2}{*}{$\begin{array}{l}\text { Group } \\
\text { Name }\end{array}$} & \multirow[b]{2}{*}{$\begin{array}{l}\text { Number } \\
\text { BAFGK }\end{array}$} & \multicolumn{3}{|c|}{ Values } & \multicolumn{3}{|c|}{ Axes } & \multicolumn{3}{|c|}{ Angles } \\
\hline & & $\begin{array}{c}U \\
\left(\mathrm{~km} \mathrm{~s}^{-1}\right)\end{array}$ & $\begin{array}{c}V \\
\left(\mathrm{~km} \mathrm{~s}^{-1}\right)\end{array}$ & $\begin{array}{c}W \\
\left(\mathrm{~km} \mathrm{~s}^{-1}\right)\end{array}$ & $\begin{array}{c}A \\
\left(\mathrm{~km} \mathrm{~s}^{-1}\right)\end{array}$ & $\begin{array}{c}B \\
\left(\mathrm{~km} \mathrm{~s}^{-1}\right)\end{array}$ & $\begin{array}{c}C \\
\left(\mathrm{~km} \mathrm{~s}^{-1}\right)\end{array}$ & $\begin{array}{c}U V \\
(\mathrm{rad})\end{array}$ & $\begin{array}{l}U W \\
(\mathrm{rad})\end{array}$ & $\begin{array}{l}V W \\
(\mathrm{rad})\end{array}$ \\
\hline$\epsilon$ Cha & 17 & -10.9 & -20.4 & -9.9 & 0.8 & 1.3 & 1.4 & 0 & 0 & 0 \\
\hline$\eta$ Cha & 6 & -10.2 & -20.7 & -11.2 & 0.2 & 0.1 & 0.1 & 0 & 0 & 0 \\
\hline TW Hya & 7 & -10.954 & -18.036 & -4.846 & 3.043 & 2.332 & 1.703 & 0.227 & 0.022 & 0.098 \\
\hline$\beta$ Pic & 34 & -10.522 & -15.964 & -9.2 & 3.167 & 2.039 & 1.609 & 0.020 & 0.045 & 0.238 \\
\hline 32 Ori & 12 & -11.8 & -18.5 & -8.9 & 0.4 & 0.4 & 0.3 & 0 & 0 & 0 \\
\hline Octans & 22 & -13.673 & -4.8 & -10.927 & 1.749 & 1.678 & 1.029 & 0.32 & -0.52 & 0.241 \\
\hline Tuc-Hor & 63 & -9.802 & -20.883 & -1.023 & 4.01 & 2.883 & 1.458 & -0.042 & -0.588 & 0.568 \\
\hline Columba & 52 & -12.311 & -21.681 & -5.694 & 2.321 & 1.43 & 1.322 & 0.470 & -0.142 & 0.329 \\
\hline Carina & 22 & -10.691 & -22.582 & -5.746 & 1.763 & 0.532 & 0.178 & 0.341 & 0.092 & 0.044 \\
\hline Argus & 38 & -22.133 & -12.122 & -4.324 & 1.992 & 1.755 & 0.774 & -0.088 & -0.026 & 0.002 \\
\hline AB Dor & 86 & -7.031 & -27.241 & -13.983 & 2.136 & 1.929 & 1.859 & 0.041 & 0.050 & 0.182 \\
\hline Car-Near & 10 & -27.020 & -18.255 & -3.021 & 3.044 & 1.819 & 1.147 & 0.023 & 0.149 & -0.286 \\
\hline Coma Ber & 104 & -2.512 & -5.417 & -1.204 & 1.868 & 1.364 & 1.876 & 0.057 & 0.106 & -0.202 \\
\hline Ursa Major & 55 & 14.278 & 2.392 & -8.987 & 2.64 & 0.594 & 0.407 & -0.799 & -0.766 & 0.500 \\
\hline$\chi^{01}$ For & 14 & -12.29 & -20.95 & -4.9 & 0.98 & 0.92 & 1.07 & 0 & 0 & 0 \\
\hline Hyades & 260 & -41.1 & -19.2 & -1.4 & 0.23 & 0.23 & 0.23 & 0 & 0 & 0 \\
\hline Field (Sirius) & 5800 & 8 & 2 & -7.25 & 12 & 6 & 9 & -0.436 & 0 & 0 \\
\hline Field (Coma1) & 4400 & -10 & -8 & -7.25 & 9 & 6 & 7 & -0.436 & 0 & 0 \\
\hline Field (Coma2) & 2700 & 15 & -18 & -7.25 & 14 & 7 & 7 & -0.436 & 0 & 0 \\
\hline Field (Hyades) & 5800 & -32 & -17 & -7.25 & 12 & 6 & 9 & 0.5 & 0 & 0 \\
\hline Field (Pleiades) & 5800 & -12 & -24 & -7.25 & 10 & 6 & 9 & -0.436 & 0 & 0 \\
\hline Field $(\zeta$ Her) & 800 & -35 & -48 & -7.25 & 14 & 6 & 8 & -0.436 & 0 & 0 \\
\hline Field & 14800 & -11.1 & -25 & -7.25 & 50 & 25 & 25 & 0 & 0 & 0 \\
\hline $\begin{array}{l}\text { Group } \\
\text { Name }\end{array}$ & & $\begin{array}{c}X \\
(\mathrm{pc})\end{array}$ & $\begin{array}{c}Y \\
(\mathrm{pc})\end{array}$ & $\begin{array}{c}Z \\
(\mathrm{pc})\end{array}$ & $\begin{array}{c}D \\
(\mathrm{pc})\end{array}$ & $\begin{array}{c}E \\
(\mathrm{pc})\end{array}$ & $\begin{array}{c}F \\
(\mathrm{pc})\end{array}$ & $\begin{array}{c}X Y \\
(\mathrm{rad})\end{array}$ & $\begin{array}{c}X Z \\
(\mathrm{rad})\end{array}$ & $\begin{array}{c}Y Z \\
(\mathrm{rad})\end{array}$ \\
\hline$\epsilon$ Cha & & 54 & -92 & -26 & 3 & 6 & 7 & 0 & 0 & 0 \\
\hline$\eta$ Cha & & 33.4 & -81 & -34.9 & 0.4 & 1 & 0.4 & 0 & 0 & 0 \\
\hline TW Hya & & 16.816 & -51.33 & 21.194 & 17.9 & 7.681 & 5.197 & -0.847 & 0.034 & 0.183 \\
\hline$\beta \mathrm{Pic}$ & & 7.075 & -3.509 & -16.277 & 30.736 & 16.323 & 7.186 & -0.06 & 0.043 & -0.337 \\
\hline 32 Ori & & -89.634 & -29.47 & -24.34 & 3.4 & 3.4 & 3.4 & 0 & 0 & 0 \\
\hline Octans & & 15.913 & -95.179 & -63.138 & 64.92 & 20.831 & 13.888 & 0.059 & -0.107 & 0.08 \\
\hline Tuc-Hor & & 5.477 & -19.146 & -35.177 & 22.83 & 13.179 & 6.713 & -0.175 & -0.043 & 0.287 \\
\hline Columba & & -27.056 & -26.369 & -31.674 & 22.794 & 24.357 & 15.479 & 0.497 & 0.074 & 0.317 \\
\hline Carina & & 18.582 & -65.598 & -21.795 & 16.127 & 12.938 & 3.63 & -0.747 & 0.27 & -0.24 \\
\hline Argus & & 16.075 & -21.027 & -3.39 & 28.119 & 25.709 & 6.084 & -0.434 & -0.104 & -0.018 \\
\hline AB Dor & & -4.323 & 1.391 & -17.372 & 23.857 & 21.531 & 15.014 & -0.16 & 0.085 & 0.221 \\
\hline Car-Near & & -2.961 & -19.919 & -1.955 & 23.641 & 10.619 & 5.037 & -1.176 & -0.532 & 1.006 \\
\hline Coma Ber & & -6.706 & -6.308 & 87.522 & 10 & 10 & 10 & 0 & 0 & 0 \\
\hline Ursa Major & & -6.704 & 10.134 & 21.622 & 1.388 & 0.763 & 0.251 & 0.559 & 0.312 & 0.682 \\
\hline$\chi^{01}$ For & & -28.3 & -46.3 & -83.4 & 2.9 & 2.9 & 2.9 & 0 & 0 & 0 \\
\hline Hyades & & -43.1 & 0.7 & -17.3 & 10 & 10 & 10 & 0 & 0 & 0 \\
\hline Field (Sirius) & & -0.18 & 2.1 & 3.27 & 120 & 120 & 120 & 0 & 0 & 0 \\
\hline Field (Coma1) & & -0.18 & 2.1 & 3.27 & 120 & 120 & 120 & 0 & 0 & 0 \\
\hline Field (Coma2) & & -0.18 & 2.1 & 3.27 & 120 & 120 & 120 & 0 & 0 & 0 \\
\hline Field (Hyades) & & -0.18 & 2.1 & 3.27 & 120 & 120 & 120 & 0 & 0 & 0 \\
\hline Field (Pleiades) & & -0.18 & 2.1 & 3.27 & 120 & 120 & 120 & 0 & 0 & 0 \\
\hline Field $(\zeta$ Her) & & -0.18 & 2.1 & 3.27 & 120 & 120 & 120 & 0 & 0 & 0 \\
\hline Field & & -0.18 & 2.1 & 3.27 & 120 & 120 & 120 & 0 & 0 & 0 \\
\hline
\end{tabular}

the moving group was traced back to the rest of the group individually. Outliers were defined as being more than $2 \sigma$ from the location of the NYMG at all times between the minimum and maximum reasonable age for the group, as collected in Table 1. As an example, Figure 15 shows the Tuc-Hor bona fide member CPD-64 17 within the confines of Tuc-Hor over the entire range of quoted ages (30-45 Myr), while Figure 16 shows the Tuc-Hor nonmember HIP 104308 nowhere near Tuc-Hor at any time. After the end of a filtering step, the moving group was recalculated with the refined member list. The process of filtering outliers was repeated until the moving group was self-consistent, which took three or fewer iterations. This reduced the bona fide list to 297 systems.

The volumes of the NYMGs near their reported times of formation are shown in Figure 17, overplotted on Figure 10. With currently available data, the final volume of $\beta$ Pic is actually smaller than calculated for our synthetic $25 \mathrm{Myr}$ old cluster (Section 3.3), which suggests that it is consistent with 

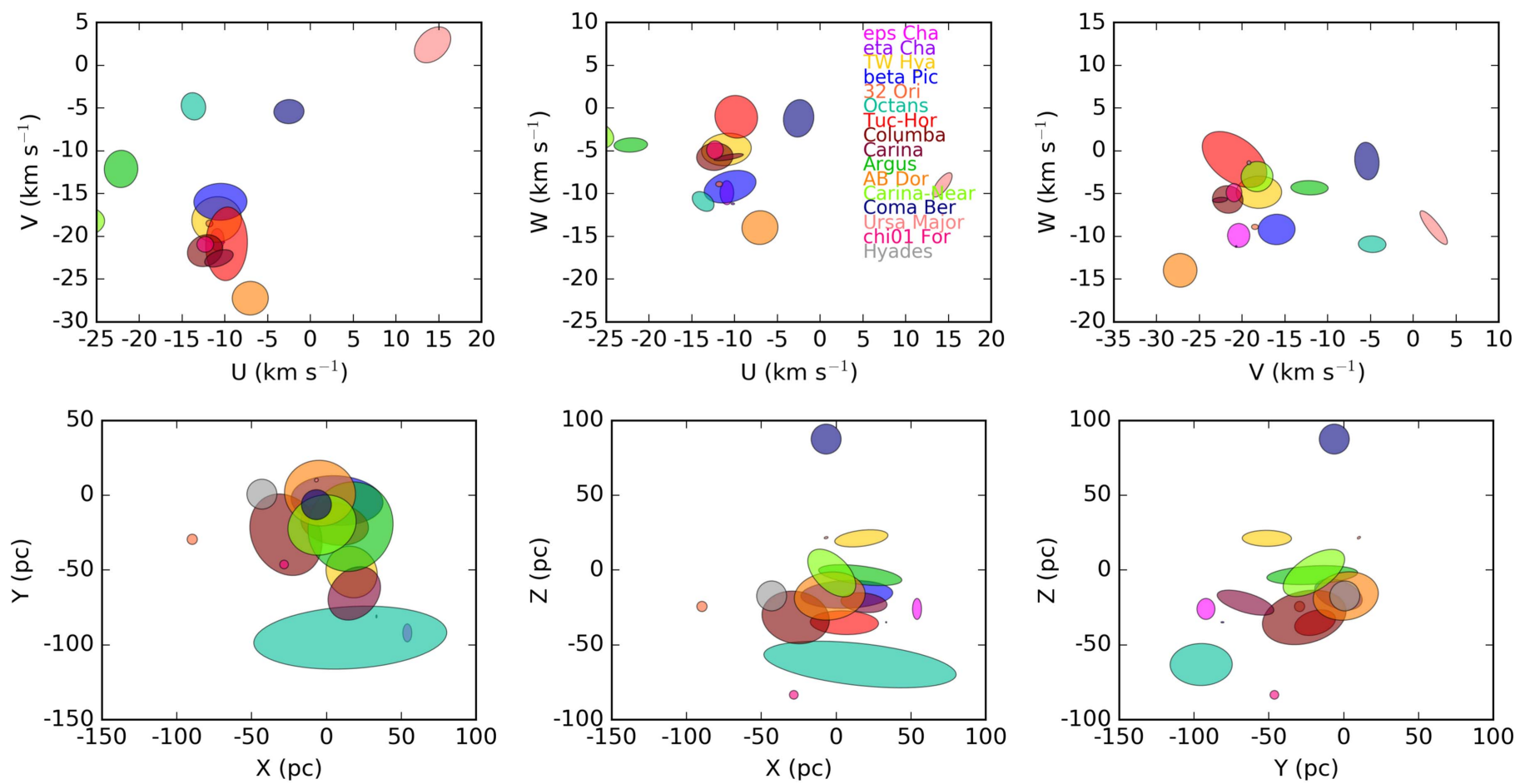

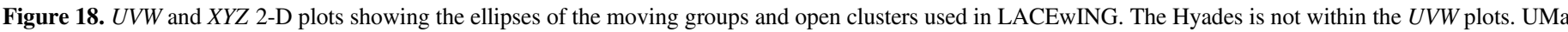

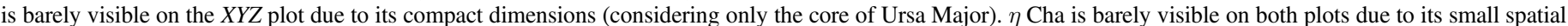
and kinematic dispersion. Note that the ellipses shown here are 2D projections of the freely oriented 3D ellipses used by LACEwING.

being a genuine product of a single burst of star formation. The same is true of AB Dor (125 Myr), suggesting that it too is consistent with being a real moving group, although $\mathrm{AB}$ Dor is also close to indistinguishable from the fake cluster of field stars (Section 3.3). The traceback results for AB Doradus in McCarthy \& Wilhelm (2014) are similar in implied volume to the TRACEwING traceback of AB Doradus in Figure 14, despite using different epicyclic parameters. This again suggests that the limiting factor in both cases is data precision.

The complete table of bona fide members (including discarded nonmembers) is given in the Catalog of Suspected Nearby Young Stars (Section 4), where they are flagged in column "Bonafide" with "B" (for bona fide system primaries), "R" (for rejected system primaries), or " $X$ " (for bona fide systems without sufficient information that could not be filtered with tracebacks or used to construct kinematic models); lower case "b," "r," and "x" flags indicate companions to the respective systems.

\subsection{Moving Group Properties}

As outlined in Section 2, the LACEwING code relies upon triaxial ellipsoid representations of the NYMGs, and an assessment of the population size. Most of the moving group ellipses were created by fitting the filtered selection of bona fide stars. The fitting routine assumes that the groups are triaxial ellipsoids with orthogonal axes. The routine finds the $U V$ plane angle with a linear fit to the projected $U V$ data, de-rotates the data to align that axis with the Cartesian plane, and then repeats the process for the $U W$ plane angle and the $V W$ plane angle. Standard deviations are fit to the de-rotated data and are taken as the axis dimensions of the ellipse. This process is repeated for 10,000 Monte Carlo iterations. The final ellipse parameters are the average of this process, and are shown in Table 7. Two- dimensional projections of the ellipsoids are shown in Figure 18.

\subsubsection{Groups Whose Properties Did Not Come from Ellipse Fitting}

Seven of the sixteen groups were not fit with our normal process. The ellipse-fitting process requires a minimum of four stars with full kinematic information. Five groups lacked sufficient numbers of stars with complete information, and their properties had to be taken instead from other sources: $\eta$ Cha (Murphy et al. 2010), $\epsilon$ Cha (Murphy et al. 2013), 32 Ori (E. Mamajek 2016, private communication), $\chi^{01}$ For (E. Mamajek 2016, private communication), and the Hyades (Röser et al. 2011). They are thus represented by axis-locked ellipses, and as shown in Table 7 , all rotation angles are set to 0 .

The properties of Coma Ber are a mix of ellipse fit to our bona fide sample for the $U V W$ space motions and converted values from van Leeuwen (2009) for the $X Y Z$ space position and tidal radius.

None of the stars in Octans have trigonometric parallaxes, but Murphy \& Lawson (2015) published estimated $U V W$ values and distances for each member. We fit ellipsoids to the $U V W$ velocities and computed $X Y Z$ position ellipses using the kinematic distances, $\alpha$, and $\delta$. Results for both Coma Ber and Octans are thus a hybrid of our work and others.

\subsubsection{The Field Population}

As shown in the top panels of Figure 19, the kinematics of the solar neighborhood as plotted from the XHIP catalog (Anderson \& Francis 2012) have a complex structure. Of the NYMGs and open clusters, only the Hyades is readily visible; the remainders of the structures are thought to be the result of Galactic resonances. 

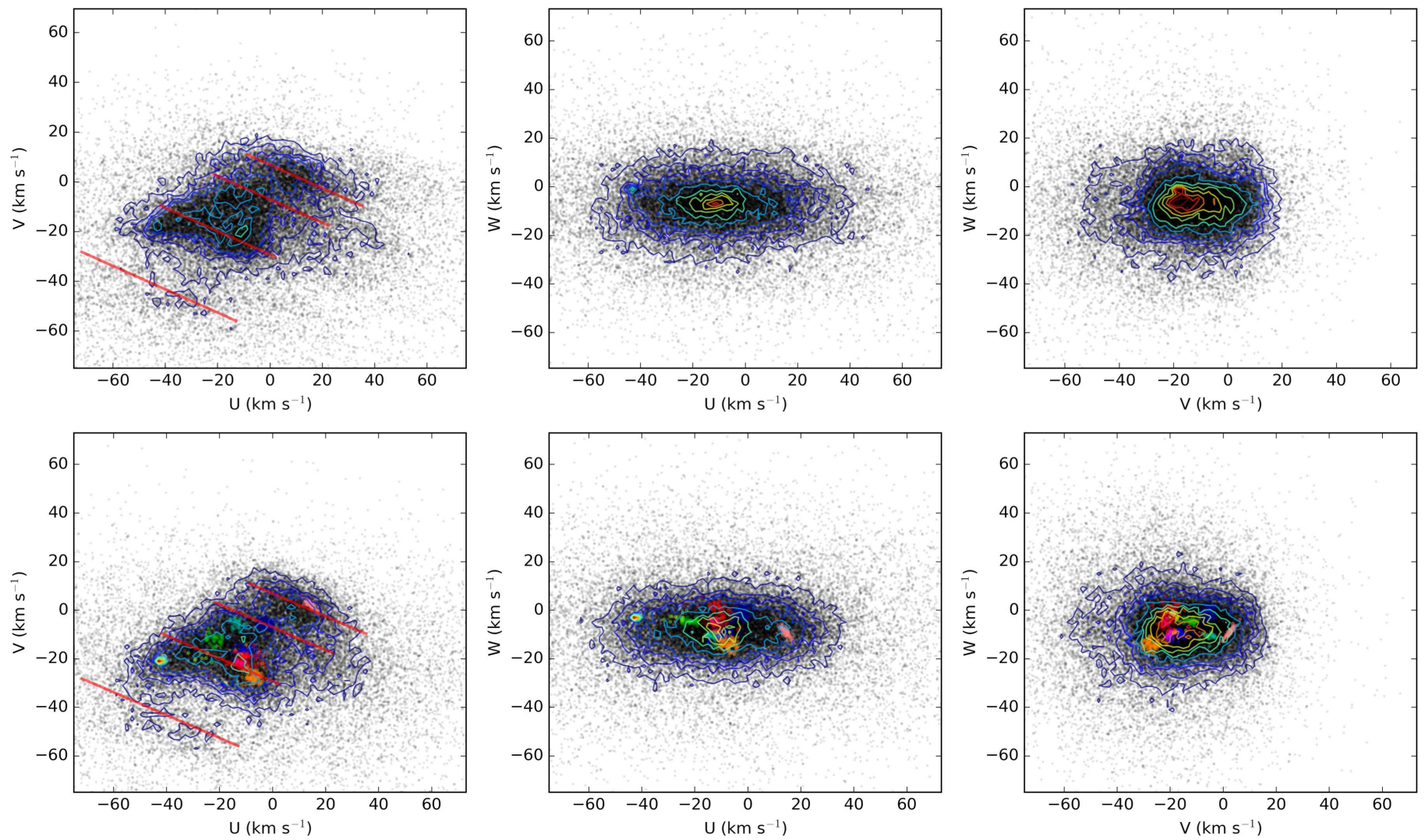

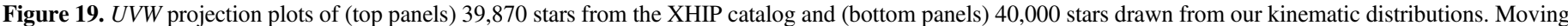

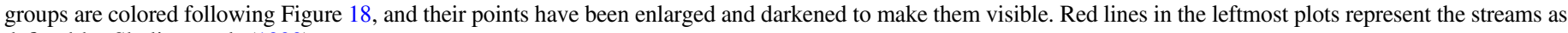
defined by Skuljan et al. (1999).

We have replicated the structures with a by-eye fit of seven ellipsoid components, which correspond to (in terms of the Skuljan et al. 1999 groups) Sirius, Coma Berenices (leading), Coma Berenices (trailing), Pleiades (leading), Hyades (trailing), $\zeta$ Hercules, and a broad generic field population. Following Skuljan et al. (1999), all groups are inclined by $25^{\circ}$ to the coordinate axes, with the exception of the Pleiades branch at $-25^{\circ}$ and the unrotated field population.

The bottom panels of Figure 19 demonstrate our synthetic field population, where the top panels plot stars with $<50 \%$ parallax uncertainties and $\gamma$ measurements with uncertainties from the XHIP catalog. Figure 20 shows the same plot for the $X Y Z$ population, which we have modeled in $X$ and $Y$ as a uniform distribution truncated at a distance of $120 \mathrm{pc}$ (to accommodate groups like Octans and $\epsilon$ Cha that extend beyond $100 \mathrm{pc}$ ), and in $Z$ as an exponential with a scale height of 300 pc, again truncated at a distance of $120 \mathrm{pc}$.

\subsection{Relative Populations of Groups}

In order to provide an appropriate simulation of members, we must also consider the relative populations of the groups. For these purposes we returned to the Catalog of Suspected Nearby Young Stars (Section 4) and considered all members of the groups, beyond just the groups that survived our bona fide vetting process above.

There are two major sources of incompleteness that must be considered here. First, the more recently discovered groups and older groups (where stars are less obviously youthful) have not been searched as completely for low-mass stars as younger and longer-known groups. For example, none of the 14 known members of the rarely studied $\chi^{01}$ For group ( $\left.500 \mathrm{Myr}\right)$ have $\mathrm{M}$ dwarf primaries, but 31 of the 38 known members of TW Hya ( $10 \mathrm{Myr}$ ) have M dwarf primaries. Second, the continued reliance on the highly magnitude-limited Hipparcos and Tycho- 2 catalogs makes it hard to identify members of more distant groups.

To account for this bias, we count only the B, A, F, G, and K primaries (and evolved versions of the same) when tallying membership in these groups. This relies upon three further assumptions. First, that all the groups are similarly complete down to spectral type $\mathrm{K} 7$; this will cause more rarely studied groups like 32 Ori and $\chi^{01}$ For to be underpredicted. Second, that the initial mass function of all of these groups has the same slope; this will overpredict membership in groups with known top-heavy mass functions like the Hyades (Goldman et al. 2013), and in theory underpredict membership in any group with a bottom-heavy mass function. Third, that a K7 spectral type refers to the same mass at the age of every group. This is harder to quantify given the differences in evolutionary tracks between different stellar evolution codes, but should cause younger groups (whose members will be cooler) like TW Hya and $\beta$ Pic to be slightly underpredicted relative to older groups.

For the field star population, we analyze the Catalog of Suspected Nearby Young Stars for lithium-rich (see Section 5.4) and bona fide members, and find that 271 systems that are bona fide or lithium rich are predicted to be within 25 pc of the Sun. Given the density of star systems within 5 pc (52 systems) there should be 6500 systems within $25 \mathrm{pc}$ of the Sun (a sample which is highly incomplete, with only 2184 systems 

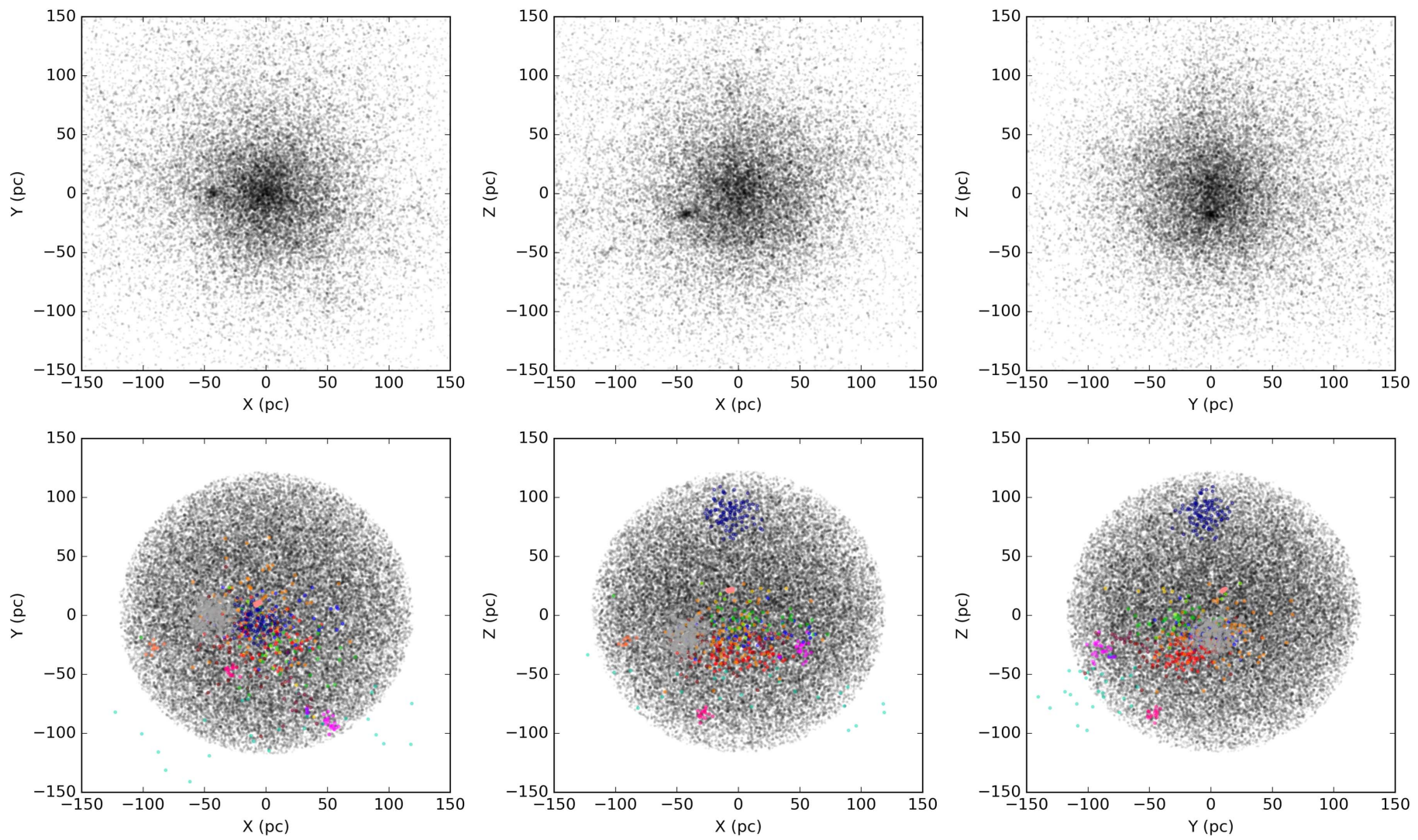

Figure 20. Same as Figure 19 except with $X Y Z$ projection plots.

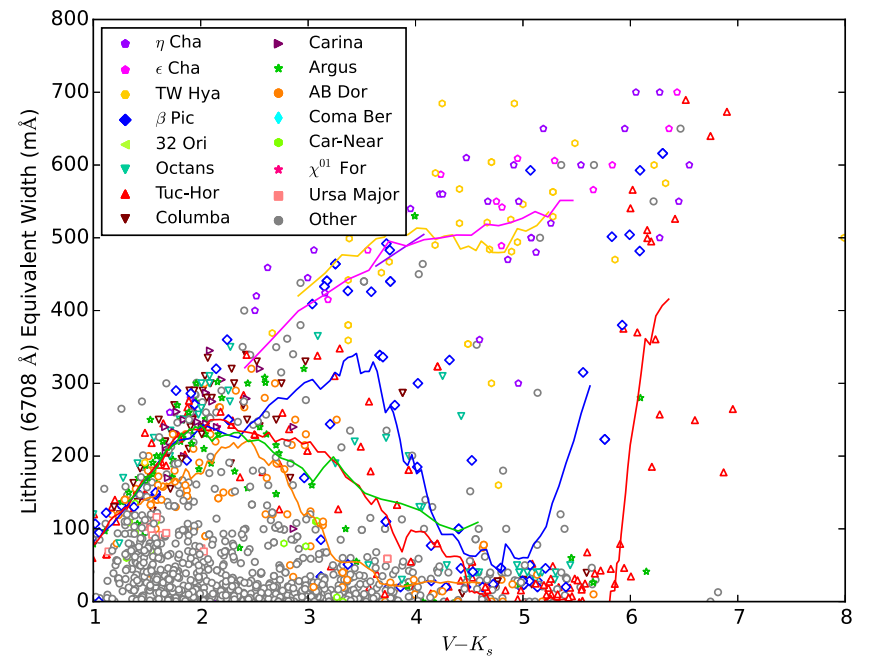

Figure 21. EW(Li) from the Catalog of Suspected Nearby Young Stars. Lines are fifteen-element moving averages.

currently known according to T. J. Henry et al. 2016, in preparation), a ratio of just under $24: 1$. We therefore take the ratio of young star systems to field stars to be 25:1. Given the further result (see Section 7.23) that less than half of the young stars are in moving groups, we take the ratio of young field stars to moving group and open cluster members to be $1: 1$. The ratio of all field stars (young and old) to moving group members is therefore 50:1. The population of field stars (split across the seven subgroups, Section 5.2.2) is thus set at 50 times the combined number of all moving group and open cluster members.

\subsection{Lithium Sample}

As a proof of concept, we use LACEwING on a sample of stars with detectable lithium. Given that kinematics and activity alone are not sufficient to say that an object is young, our process of examining only spectroscopically young objects reduces the possibility that we are attempting to reproduce erroneous membership assignments.

The presence of lithium (specifically the red-optical $6708 \AA$ doublet) is one of the most reliable spectroscopic methods of identifying young stars (Soderblom 2010). Lithium is fused at lower temperatures than hydrogen, and nearly all of it is primordial. Any amount of lithium present in a stellar spectrum (unless the object is an asymptotic giant branch star) is a sign that the object has not fused it yet, either because the object is very young, or because it is a $<60 M_{\text {Jup }}$ brown dwarf that never reaches sufficiently high temperatures (Rebolo et al. 1992). The exact age at which lithium is depleted varies based on the mass of the object (Yee \& Jensen 2010) and spans ages typical of the NYMGs. In Figure 21, we plot all lithium measurements from the Catalog of Suspected Nearby Young Stars, with fifteen-element moving averages tracing out the lithium depletion as a function of age. At the age of $\epsilon$ Cha, barely any lithium is gone, while by the age of $\mathrm{AB}$ Dor, there is a very clear lithium depletion.

Although our survey of papers reporting lithium is not complete, 1877 of the 5350 stars in our catalog of suspected young stars (Section 4) have at least one attempt to measure their $\mathrm{Li}$ I $\lambda 6707.8 \AA \mathrm{EW}$ doublet $(\mathrm{EW}(\mathrm{Li}))$, as shown in Figure $21 . \mathrm{EW}(\mathrm{Li})$ is frequently reported without uncertainties on the measurements. We have created a lithium sample by selecting objects with $\mathrm{EW}(\mathrm{Li})>\max \left(10 \mathrm{~m} \AA, 2 \sigma_{\mathrm{Li}}\right)$ from the 
Catalog of Suspected Nearby Young Stars (Section 4). Where no uncertainty is quoted, the uncertainty is set at $50 \mathrm{~m} \AA$. This matches the limits of several major papers reporting lithium, including Eisenbeiss et al. (2013), Kraus et al. (2014b), and Rodriguez et al. (2013); the largest upper limits reported by Malo et al. (2013) and Moór et al. (2013) are $46 \mathrm{~mA}$.

Objects that are companions (according to the WDS and other resources in Section 4) to stars in the lithium sample are also included, yielding a total of 1179 stars. From there, we considered only the primaries of the systems that have at least one lithium-detected member (including 15 primaries that do not themselves have measured lithium) so as not to count star systems more than once, resulting in a list of 1037 star systems.

Objects that were members of groups not within $100 \mathrm{pc}$ were removed. This includes the members of IC 2391 identified by Torres et al. (2008, p. 757) as members of Argus, and a wide variety of objects from Guenther et al. (2007) that belong to more distant regions (e.g., Chamæleon, $\rho$ Ophiuchi, ScorpiusCentaurus). This reduced the size of the lithium sample to 930 systems.

This sample substantially overlaps with the bona fide sample (Section 5.1): 152 systems are in both lists. Some genuinely young stars at the fully convective boundary around spectral type M3 V are excluded from this sample because lithium is fully depleted at their ages, while some more massive stars still maintain lithium even at old ages (e.g., $\alpha$ Cen AB; Mishenina et al. 2012). The sample can be found in the "LiSample" column of the Catalog of Suspected Nearby Young Stars, where system primaries that qualify for the lithium sample are flagged with " $L$," system primaries that qualify for the lithium sample but have membership in a more distant group are flagged with " $F$," and primaries that do not have lithium but have a companion that qualifies are flagged with "A." As with the bona fide sample, lowercase letters follow the same rules but denote companions.

\section{LACEwING Performance Comparison}

In this section we present the results of running LACEwING on the bona fide and lithium samples (Section 5.4) and compare its performance (and algorithm) to other available moving group identification codes.

\subsection{Code and Methodology Comparison}

\subsubsection{Public Codes}

LACEwING does computations in observation space, directly comparing the proper motion and radial velocity vectors. Like BANYAN II, LACEwING treats groups as sixdimensional freely oriented ellipsoids and has a separate set of calibrations for use when stars are already known to be young. Unlike BANYAN II, groups have different population sizes relative to each other, and the field population to young star ratio is 25:1, rather than 4.1:1 as stated by Gagné et al. (2014b).

Each publicly available code for finding moving groups uses a different algorithm and is based on different bona fide member lists.

$B A N Y A N^{11}$ - (Malo et al. 2013). It uses Bayesian statistical techniques to provide an assessment of the moving group membership of a target object. It works in observation space,

\footnotetext{
11 http://www.astro.umontreal.ca/ malo/banyan.php, checked 2016 October 7 .
}

directly testing against predictions of $\alpha, \delta, \mu_{\alpha}, \mu_{\delta}, \pi$, and $\gamma$ (a version also exists that considers $I_{\text {Cousins }}-J$ photometry, but this is not publicly available) to identify objects based on both position and motion, with an additional component comparing $X Y Z$ positions. It has models that test for TW Hya, $\beta$ Pic, TucHor, Columba, Carina, Argus, AB Dor, and a Field (Old) population. The output probability is relative to all other groups, such that all percentages sum to $100 \%$.

BANYAN $I I^{12}$ - Gagné et al. (2014b). It is a modification of BANYAN with new kinematic models and methods of treating them. It works in observational space, and uses $\alpha, \delta, \mu_{\alpha}, \mu_{\delta}, \pi$, and $\gamma$ to determine matches based on $U V W$ space velocities and $X Y Z$ space positions. Like BANYAN, a version of BANYAN II exists that considers $J-K_{S}$ photometry, but is not publicly available. It has models that evaluate membership in TW Hya, $\beta$ Pic, Tuc-Hor, Columba, Carina, Argus, AB Dor, a young field, and an old field, where the field is based on a Besançon model (Robin et al. 2003). Unlike BANYAN, the kinematic models of the moving groups are represented as freely oriented three-dimensional ellipsoids rather than axis-locked moving groups. Also unlike BANYAN, the field population is expected to outnumber field objects at a rate of 4.1:1, although all moving groups are expected to be the same relative size as each other. BANYAN II has separate probability measurements if the object is known to be young.

Rodriguez convergence code ${ }^{13}-$ Rodriguez et al. (2013) uses the convergent points of six groups (TW Hya, $\beta$ Pic, Chameleon-Near, Tuc-Hor, Columba, and AB Dor) and their scalar velocities to predict memberships based on $\alpha, \delta, \mu_{\alpha}$, and $\mu_{\delta}$. Its probabilities are based on a mathematical comparison of the expected proper motion vector to a computed one. While the code predicts Dist and $\gamma$, it does not use either value in computations.

\subsubsection{Other Codes}

The convergence method used in Montes et al. (2001b) is an implementation of techniques used by Eggen (1995). It uses the velocity contributions $V_{\text {tangential }}=4.74 \mu \pi^{-1}$ and $V_{\text {radial }}$ to determine the total velocity $V_{\text {Total. }}$. Using the angular separation between the star and the convergent point $(\lambda)$, it splits $V_{\text {Total }}$ into velocity components parallel $\left(V_{\mathrm{T}}\right)$ and perpendicular to (PV) the convergent vector. It also splits the parallel velocity vector $V_{\mathrm{T}}$ into an $\mathrm{RV}$ component $\rho_{\mathrm{C}}$. The two metrics of membership reported are whether $\mathrm{PV}<0.1 V_{\mathrm{T}}$ and whether $\rho_{\mathrm{C}}$ and $V_{\text {radial }}$ differ by less than $4-8 \mathrm{~km} \mathrm{~s}^{-1}$-no percentages are calculated. As used in Montes et al. (2001b), the code considers membership in the Local Association, Hyades Supercluster, IC 2391 Supercluster, Ursa Major moving group, and Castor moving group.

The convergent point method used by Mamajek (2005) is much more similar to the Rodriguez convergence code.

The codes used by Lépine \& Simon (2009), Schlieder et al. (2010, 2012b, 2012c), and Kraus et al. (2014a) are quite similar to LACEwING. They take the $U V W$ velocity of a moving group and determine the expected proper motion and radial velocity of a member at those equatorial coordinates (at an implied distance of $1 \mathrm{pc}$ ); the proper motion vector is then used to determine a kinematic distance and kinematic RV. These

\footnotetext{
12 http://www.astro.umontreal.ca/ gagne/banyanII.php, checked 2016 October 7 .

13 http://dr-rodriguez.github.io/CPCalc.html, checked 2016 October 7.
} 
kinematic distances are compared to spectroscopic, photometric, or trigonometric distances (Lépine \& Simon 2009), or with spectrophotometric distances and a distance modulus cutoff to enforce the stars falling on the approximately correct isochrone (Kraus et al. 2014a). They lack the $X Y Z$ position metric used by LACEwING, and do not convert the proper motion matches or distance matches to membership probabilities. Lépine \& Simon (2009) only reports members of $\beta$ Pic; Schlieder et al. (2010, 2012b, 2012c) report $\beta$ Pic and AB Dor; Kraus et al. (2014a) only considers Tuc-Hor. It is not clear if the codes consider more than one or two groups in their studies.

The moving group identification techniques used by Shkolnik et al. (2012), Riedel et al. (2014; see also Baines et al. 2012), and Binks et al. (2015) are quite similar, and compute proximity to the $U V W$ distributions of stars using the formula

$$
\begin{aligned}
\chi^{2}= & \frac{1}{3}\left(\frac{\left(U_{\mathrm{star}}-U_{\mathrm{NYMG}}\right)^{2}}{\sigma_{U, \mathrm{star}}^{2}+\sigma_{U, \mathrm{NYMG}}^{2}}\right. \\
& +\frac{\left(V_{\mathrm{star}}-V_{\mathrm{NYMG}}\right)^{2}}{\sigma_{V, \mathrm{star}}^{2}+\sigma_{V, \mathrm{NYMG}}^{2}} \\
& \left.+\frac{\left(W_{\mathrm{star}}-W_{\mathrm{NYMG}}\right)^{2}}{\sigma_{W, \mathrm{star}}^{2}+\sigma_{W, \mathrm{NYMG}}^{2}}\right),
\end{aligned}
$$

which computes the difference between the star's $U V W$ and the moving group's $U V W$ in units roughly analogous to standard deviations. This technique does not deal with missing information as easily as a convergence-style routine; it is impossible to calculate the $U V W$ without full kinematic information, requiring the use of estimates or repeated calculations over a range. Differences are mostly in implementation. Shkolnik et al. (2012) studied 14 NYMGs and assumed a $2 \mathrm{~km} \mathrm{~s}^{-1}$ velocity dispersion for all groups; they required a maximum $\chi^{2}$ value of six and a maximum velocity difference (to avoid identifying stars with large uncertainties) of $5 \mathrm{~km} \mathrm{~s}^{-1}$. They calculated photometric distances where parallaxes were not available; all targets had $\gamma$. Binks et al. (2015) studied 10 groups with individualized dispersions and set a $\chi^{2}$ cutoff of 3.78 , and had a maximum velocity difference of $5 \mathrm{~km} \mathrm{~s}^{-1}$. Riedel et al. (2014) studied 13 groups with individualized dispersions, and accepted a maximum $\chi^{2}$ (there, called $\gamma$ ) value of 4.0. There was no limit on actual velocity difference. Where radial velocity did not exist, they computed $U V W$ values for a range of radial velocities from -100 to $+100 \mathrm{~km} \mathrm{~s}^{-1}$ and took the minimum $\chi^{2}$ (and corresponding $\mathrm{RV}$ ) from a polynomial fit as the correct value; all targets had parallaxes.

The kinematic moving group identification technique in the SACY papers (e.g., Torres et al. 2008, p. 757) is similar to, but simpler than the Shkolnik et al. (2012), Riedel et al. (2014), and Binks et al. (2015) method, with $F=(p \times$ $\left(M_{\mathrm{v}}-M_{\mathrm{v}, \text { iso }}\right)^{2}+\left(U_{\mathrm{star}}-U_{\mathrm{NYMG}}\right)^{2}+\left(V_{\mathrm{star}}-V_{\mathrm{NYMG}}\right)^{2}+$ $\left.\left(W_{\text {star }}-W_{\text {NYMG }}\right)^{2}\right)^{\frac{1}{2}}$, where $M_{\mathrm{v}}-M_{\mathrm{v} \text {,iso }}$ is the difference between the absolute $V$ magnitude of the star and the absolute $V$ magnitude of a star of a particular $V-I_{C}$ color, on the ageappropriate isochrone, and $F$ is the velocity-space separation between a star (utilizing a best-fit $U V W$ for the star if the distance is unknown) and a moving group. In practice, they claim that the scaling constant $p$ was only non-zero for the Octans moving group, and therefore the equation is generally a simple velocity separation independent of uncertainties. The cutoff of good values starts at $F=3.5$, but the process used by SACY is to iteratively minimize $U V W$ for a cluster of young stars until all outliers are removed. The team has considered nine groups: $\beta$ Pic, Tuc-Hor, Columba, Carina, TW Hya, $\epsilon$ Cha, Octans, Argus, and AB Dor.

The code used by Klutsch et al. (2014) considers membership in two ways: first, a method using Gaussian representations of the $U V W$ velocities (and their errors, formed by error propagation); second, member-to-member analysis, wherein the stars are compared to both the moving group center and the other known members; this allows for more variation and is potentially more robust at dealing with non-Gaussian distributions of stars.

\subsection{Performance Comparison}

We present the results of comparing LACEwING to the BANYAN, BANYAN II, and Convergence codes in Table 8. For each group, we record the number of members in our bona fide sample (Column 2), the number of objects identified by the code as members of that group (Column 3), and the number of objects identified as members that were genuine members (Column 4), with the corresponding number of false positives (Column 5).

For instance, LACEwING (in young star mode) identifies 24 $\epsilon$ Cha members in the bona fide sample, and all 24 of its recovered members are known members of $\epsilon$ Cha: a $0 \%$ false positive rate, but not a $100 \%$ recovery rate given that there were 27 bona fide members of $\epsilon$ Cha to find. In contrast, LACEwING (in both modes) did recover all 11 bona fide members of $\chi^{01}$ For, and did not identify other stars as $\chi^{01}$ For members.

Looking at false positive rates is not as useful for the lithium sample (Table 9), because 518 out of the 930 lithium-rich stars are not previously known members of moving groups and many are likely to actually be members that have not been investigated kinematically before.

The comparisons in Tables 8 and 9 are not "recoveries" in the traditional sense, as there are no widely accepted correct answers (apart from, perhaps, the bona fide member list). LACEwING, BANYAN, and BANYAN II are roughly tied in terms of accuracy, except with the AB Dor moving group, where BANYAN clearly outperforms all other codes.

Tests of LACEwING while under development show that as more groups are added to the kinematic model, the recovery rate drops, but the false positive rate drops as well. While developing LACEwING, attempts were made to strike a compromise between recovery rates and false positive rates.

\section{The NYMGs}

In the course of this study, we have collected notes about the moving groups themselves, both generally and in terms of what our TRACEwING and LACEwING analyses say about the existence and properties of the moving groups themselves.

\section{1. $\epsilon$ Chamaeleontis}

This group was discovered by Mamajek et al. (2000) during examination of the $\eta$ Cha open cluster. For a time, it was assumed (e.g., Torres et al. 2008, p. 757) to be co-eval with the 
Table 8

Comparison of Completion in the Bona Fide Data Sets

\begin{tabular}{|c|c|c|c|c|c|}
\hline $\begin{array}{l}\text { Group } \\
\text { (1) }\end{array}$ & $\begin{array}{l}\text { Real } \\
(2)\end{array}$ & $\begin{array}{l}\text { Identified } \\
\text { (3) }\end{array}$ & $\begin{array}{c}\text { Recovered } \\
\text { (4) }\end{array}$ & $\begin{array}{l}\text { False Positive } \\
\text { (5) }\end{array}$ & $\begin{array}{l}\text { Code } \\
(6)\end{array}$ \\
\hline \multirow[t]{2}{*}{$\overline{\epsilon \text { Cha }}$} & 27 & 24 & 24 & 0 & L-Y \\
\hline & & 22 & 22 & 0 & L-F \\
\hline \multirow[t]{2}{*}{$\eta$ Cha } & 2 & 2 & 2 & 0 & L-Y \\
\hline & & 2 & 2 & 0 & L-F \\
\hline \multirow[t]{6}{*}{ TW Hya } & 17 & 15 & 15 & 0 & L-Y \\
\hline & & 10 & 10 & 0 & L-F \\
\hline & & 16 & 16 & 0 & B1 \\
\hline & & 16 & 16 & 0 & B2-Y \\
\hline & & 16 & 16 & 0 & B2-F \\
\hline & & 19 & 6 & 13 & $\mathrm{C}$ \\
\hline \multirow[t]{6}{*}{$\beta \mathrm{Pic}$} & 28 & 22 & 22 & 0 & L-Y \\
\hline & & 14 & 14 & 0 & L-F \\
\hline & & 43 & 27 & 16 & B1 \\
\hline & & 25 & 25 & 0 & B2-Y \\
\hline & & 25 & 25 & 0 & B2-F \\
\hline & & 38 & 14 & 24 & $\mathrm{C}$ \\
\hline \multirow[t]{2}{*}{32 Ori } & 10 & 9 & 9 & 0 & L-Y \\
\hline & & 8 & 8 & 0 & L-F \\
\hline \multirow[t]{2}{*}{ Octans } & 45 & 44 & 44 & 0 & L-Y \\
\hline & & 17 & 17 & 0 & L-F \\
\hline \multirow[t]{6}{*}{ Tuc-Hor } & 32 & 30 & 29 & 1 & L-Y \\
\hline & & 31 & 30 & 1 & L-F \\
\hline & & 30 & 29 & 1 & B1 \\
\hline & & 31 & 30 & 1 & B2-Y \\
\hline & & 31 & 30 & 1 & B2-F \\
\hline & & 70 & 28 & 42 & $\mathrm{C}$ \\
\hline \multirow[t]{6}{*}{ Columba } & 16 & 14 & 13 & 1 & L-Y \\
\hline & & 10 & 10 & 0 & L-F \\
\hline & & 29 & 15 & 14 & B1 \\
\hline & & 15 & 14 & 1 & B2-Y \\
\hline & & 15 & 14 & 1 & B2-F \\
\hline & & 34 & 8 & 26 & $\mathrm{C}$ \\
\hline \multirow[t]{5}{*}{ Carina } & 4 & 4 & 4 & 0 & L-Y \\
\hline & & 3 & 3 & 0 & L-F \\
\hline & & 4 & 4 & 0 & B1 \\
\hline & & 7 & 4 & 3 & B2-Y \\
\hline & & 7 & 4 & 3 & B2-F \\
\hline \multirow[t]{5}{*}{ Argus } & 6 & 8 & 5 & 3 & L-Y \\
\hline & & 5 & 5 & 0 & L-F \\
\hline & & 8 & 6 & 2 & B1 \\
\hline & & 7 & 6 & 1 & B2-Y \\
\hline & & 7 & 6 & 1 & B2-F \\
\hline \multirow[t]{6}{*}{ AB Dor } & 35 & 33 & 30 & 3 & L-Y \\
\hline & & 26 & 26 & 0 & L-F \\
\hline & & 35 & 35 & 0 & B1 \\
\hline & & 30 & 30 & 0 & B2-Y \\
\hline & & 30 & 30 & 0 & B2-F \\
\hline & & 74 & 28 & 46 & $\mathrm{C}$ \\
\hline \multirow[t]{2}{*}{ Car-Near } & 9 & 3 & 3 & 0 & L-Y \\
\hline & & 2 & 2 & 0 & L-F \\
\hline \multirow[t]{2}{*}{ Coma Ber } & 45 & 33 & 33 & 0 & L-Y \\
\hline & & 31 & 31 & 0 & L-F \\
\hline \multirow[t]{2}{*}{ Ursa Major } & 5 & 5 & 5 & 0 & L-Y \\
\hline & & 5 & 5 & 0 & L-F \\
\hline
\end{tabular}

Table 8

(Continued)

\begin{tabular}{lccccc}
\hline \hline $\begin{array}{l}\text { Group } \\
(1)\end{array}$ & $\begin{array}{c}\text { Real } \\
(2)\end{array}$ & $\begin{array}{c}\text { Identified } \\
(3)\end{array}$ & $\begin{array}{c}\text { Recovered } \\
(4)\end{array}$ & $\begin{array}{c}\text { False Positive } \\
(5)\end{array}$ & $\begin{array}{l}\text { Code } \\
(6)\end{array}$ \\
\hline$\chi^{01}$ For & 11 & 11 & 11 & 0 & L-Y \\
& & 10 & 10 & 0 & L-F \\
\hline Hyades & 0 & 0 & 0 & 0 & L-Y \\
& & 0 & 0 & 0 & L-F \\
\hline
\end{tabular}

Note. Comparison of the recovery rates of LACEwING in Young star (L-Y) and Field star (L-F) calibration to BANYAN (B1), BANYAN II in Young star (B2-Y) and Field star (B2-F) calibration, and the Convergence (C) code. In this context, "membership" means the highest probability was for the group in question.

open cluster and likely part of the same star-forming event; Murphy et al. (2013) recently used evolutionary models that suggest it is younger than $\eta$ Cha. The hottest (and likely most massive) member of $\epsilon$ Cha is $\epsilon$ Cha itself, a B9V star.

\section{2. $\eta$ Chamaleontis Open Cluster}

First discovered by Mamajek et al. (1999), $\eta$ Cha is the smallest and third-closest open cluster to the Sun. The hottest member of the open cluster is $\eta$ Cha itself, a B9V star whose companions form most of the members of the open cluster. Only two members of $\eta$ Cha ( $\eta$ Cha and RS Cha) have parallaxes, so our moving group parameters are taken from Murphy et al. (2010).

\subsection{TW Hydrae}

Discovered by de la Reza et al. (1989), TW Hya was the second known NYMG discovered (after Ursa Major), and TW Hya itself is the closest classical T Tauri star to the Sun. Its $U V W$ velocity and projected sky position are similar to the Lower Centaurus Crux region of the Sco-Cen star-forming complex, and the group itself extends from roughly $30 \mathrm{pc}$ from the Sun to the near edge of Lower Centaurus Crux. Many proposed TW Hya members are now thought to actually be part of that background group. The hottest member of TW Hya is not actually TW Hya itself (K6Ve), but TWA 11 (HR 4796), an A0V star.

\subsection{Orionis}

This group was first noticed by Mamajek (2007) as a small knot of stars around the B5V+B7V binary star 32 Ori; very little has been studied about this group of stars. Where most of the NYMGs have been linked to origins in the Sco-Cen starforming region, 32 Ori may have a different origin: Bouy \& Alves (2015) proposed a different arrangement of gas near the Sun where instead of Gould's belt, there are three parallel streams, one of which would stretch from 32 Ori to the Orion Nebula Complex.

\section{5. $\beta$ Pictoris}

Discovered by Barrado y Navascués et al. (1999b), $\beta$ Pic is one of the moving groups that effectively surrounds the Sun. The most massive member of the moving group is HR 6070 (A0V), though our tracebacks actually rejected it as a member. 
Table 9

Comparison of Completion in the Lithium Data Sets

\begin{tabular}{|c|c|c|c|c|c|}
\hline $\begin{array}{l}\text { Group } \\
\text { (1) }\end{array}$ & $\begin{array}{l}\text { Real } \\
(2)\end{array}$ & $\begin{array}{l}\text { Identified } \\
\text { (3) }\end{array}$ & $\begin{array}{c}\text { Recovered } \\
\text { (4) }\end{array}$ & $\begin{array}{l}\text { False Positive } \\
\text { (5) }\end{array}$ & $\begin{array}{l}\text { Code } \\
(6)\end{array}$ \\
\hline \multirow[t]{2}{*}{$\epsilon$ Cha } & 30 & 30 & 25 & 5 & L-Y \\
\hline & & 27 & 24 & 3 & L-F \\
\hline \multirow[t]{2}{*}{$\eta$ Cha } & 15 & 17 & 14 & 3 & L-Y \\
\hline & & 15 & 14 & 1 & L-F \\
\hline \multirow[t]{6}{*}{ TW Hya } & 31 & 28 & 25 & 3 & L-Y \\
\hline & & 11 & 11 & 0 & L-F \\
\hline & & 34 & 27 & 7 & B1 \\
\hline & & 28 & 27 & 1 & B2-Y \\
\hline & & 28 & 27 & 1 & B2-F \\
\hline & & 65 & 12 & 53 & $\mathrm{C}$ \\
\hline \multirow[t]{6}{*}{$\beta \mathrm{Pic}$} & 44 & 24 & 19 & 5 & L-Y \\
\hline & & 13 & 12 & 1 & L-F \\
\hline & & 77 & 36 & 41 & B1 \\
\hline & & 38 & 26 & 12 & B2-Y \\
\hline & & 37 & 26 & 11 & B2-F \\
\hline & & 78 & 20 & 58 & $\mathrm{C}$ \\
\hline \multirow[t]{2}{*}{32 Ori } & 0 & 1 & 0 & 1 & L-Y \\
\hline & & 0 & 0 & 0 & L-F \\
\hline \multirow[t]{2}{*}{ Octans } & 22 & 31 & 21 & 10 & L-Y \\
\hline & & 9 & 9 & 0 & L-F \\
\hline \multirow[t]{6}{*}{ Tuc-Hor } & 68 & 61 & 55 & 6 & L-Y \\
\hline & & 46 & 43 & 3 & L-F \\
\hline & & 54 & 52 & 2 & B1 \\
\hline & & 53 & 50 & 3 & B2-Y \\
\hline & & 53 & 50 & 3 & B2-F \\
\hline & & 94 & 49 & 45 & $\mathrm{C}$ \\
\hline \multirow[t]{6}{*}{ Columba } & 41 & 35 & 15 & 20 & L-Y \\
\hline & & 12 & 8 & 4 & L-F \\
\hline & & 70 & 32 & 38 & B1 \\
\hline & & 33 & 19 & 14 & B2-Y \\
\hline & & 33 & 19 & 14 & B2-F \\
\hline & & 79 & 14 & 65 & $\mathrm{C}$ \\
\hline \multirow[t]{5}{*}{ Carina } & 21 & 19 & 8 & 11 & L-Y \\
\hline & & 8 & 4 & 4 & L-F \\
\hline & & 17 & 8 & 9 & B1 \\
\hline & & 19 & 7 & 12 & B2-Y \\
\hline & & 19 & 7 & 12 & B2-F \\
\hline \multirow[t]{5}{*}{ Argus } & 28 & 23 & 7 & 16 & L-Y \\
\hline & & 8 & 4 & 4 & L-F \\
\hline & & 24 & 11 & 13 & B1 \\
\hline & & 12 & 6 & 6 & B2-Y \\
\hline & & 11 & 6 & 5 & B2-F \\
\hline \multirow[t]{6}{*}{ AB Dor } & 65 & 51 & 36 & 15 & L-Y \\
\hline & & 25 & 23 & 2 & L-F \\
\hline & & 62 & 50 & 12 & B1 \\
\hline & & 27 & 24 & 3 & B2-Y \\
\hline & & 25 & 24 & 1 & B2-F \\
\hline & & 139 & 48 & 91 & $\mathrm{C}$ \\
\hline \multirow[t]{2}{*}{ Car-Near } & 4 & 3 & 1 & 2 & L-Y \\
\hline & & 0 & 0 & 0 & L-F \\
\hline \multirow[t]{2}{*}{ Coma Ber } & 0 & 1 & 0 & 1 & L-Y \\
\hline & & 0 & 0 & 0 & L-F \\
\hline \multirow[t]{2}{*}{ Ursa Major } & 11 & 3 & 1 & 2 & L-Y \\
\hline & & 1 & 1 & 0 & $\mathrm{~L}-\mathrm{F}$ \\
\hline
\end{tabular}

Table 9

(Continued)

\begin{tabular}{lccccc}
\hline \hline $\begin{array}{l}\text { Group } \\
(1)\end{array}$ & $\begin{array}{c}\text { Real } \\
(2)\end{array}$ & $\begin{array}{c}\text { Identified } \\
(3)\end{array}$ & $\begin{array}{c}\text { Recovered } \\
(4)\end{array}$ & $\begin{array}{c}\text { False Positive } \\
(5)\end{array}$ & $\begin{array}{l}\text { Code } \\
(6)\end{array}$ \\
\hline$\chi^{01}$ For & 1 & 3 & 1 & 2 & L-Y \\
& & 1 & 1 & 0 & L-F \\
\hline Hyades & 0 & 11 & 0 & 11 & L-Y \\
& & 0 & 0 & 0 & L-F \\
\hline
\end{tabular}

Note. Same as Table 8, but for the lithium sample.

The hottest member of $\beta$ Pic not rejected by our tracebacks is $\beta$ Pic itself, an A5V star with a planet and prominent debris disk.

\subsection{Capricornus}

Discovered by van den Ancker et al. (2000), Capricornus had only two proposed members, one of which (BD-17 6127 $\mathrm{AB})$ is now identified as a $\beta$ Pic member; the other, HD 356823 , matches no known group. No attempt was made to consider this moving group for inclusion in LACEwING.

\subsection{Chamaeleon-Near}

First published by Zuckerman \& Song (2004), most of this moving group's members are now thought to be part of Argus and $\epsilon$ Cha. This group was never considered for inclusion in LACEwING.

\subsection{Octans}

First proposed by Torres et al. (2008, p. 757), Octans is one of the most distant moving groups. Based on our ellipse fits, it is also the largest (and therefore least dense) moving group, and LACEwING has difficulty differentiating its members from field stars. Very few papers have studied Octans, apart from Murphy \& Lawson (2015). The hottest star in Octans is HD 36968, an F2 star. Every other group except Carina and CarNear has a hotter member in the B5-A5 range, suggesting that there is either something different about Octans, or hotter members remain to be identified.

\subsection{Tucana-Horologium}

The Horologium moving group was discovered by Torres et al. (2000), followed by the Tucana moving group (Zuckerman et al. 2001), and then the realization that they were two parts of the same group (Zuckerman 2001). Thanks primarily to the work of Kraus et al. (2014a), Tuc-Hor has the most known members of any NYMG, though most are $M$ dwarfs. Considering only the BAFGK members, Tuc-Hor is still likely smaller (63 BAFGK members) than AB Dor (86 BAFGK members). The hottest star in Tuc-Hor is $\alpha$ Pav, a B2IV star, although the $\beta$ Tuc sextuple system (B9V+A0V $+\mathrm{A} 2 \mathrm{~V}+\mathrm{A} 7 \mathrm{~V}$ +unknowns) may be the most massive.

For most NYMGs, their members have generally nonexistent probabilities of membership in other NYMGs. This is not true for Tuc-Hor: a large fraction of Tuc-Hor members also have low but significant probabilities of membership in Columba, and somewhat less significant probability of membership in $\beta$ Pic. 


\subsection{Columba}

The Columba moving group was first announced by Torres et al. (2008, p. 757) along with the Carina moving group, as subdivisions of a larger "GAYA" complex that also contained Tuc-Hor. While Columba does have a different $U V W$ velocity from Tuc-Hor and Carina, its spatial location at one end of the Tuc-Hor (see Figure 13) and persistently similar age to the other two groups continue to suggest that this may not be distinct from Tuc-Hor. LACEwING finds that many Columba bona fide members have low probabilities of membership in Tuc-Hor. The most massive member of Columba is HR 1621 (B9V). The known planet host (Marois et al. 2008) and putative Columba member (Baines et al. 2012) HR 8799 survived both TRACEwING filtering and LACEwING analysis as a bona fide member of Columba, despite the fact that its high mass (as an A5V star) and position far from other known members are at odds with the idea of mass segregation.

\subsection{Carina}

As mentioned in Section 7.10, Carina was first discovered by Torres et al. (2008, p. 757) as part of the "GAYA" complex, a larger group that included Tuc-Hor and Columba; like Columba, Carina sits physically adjacent to Tuc-Hor. A preliminary version of LACEwING used in Riedel (2016b) and Faherty et al. (2016) actually excluded Carina because, with an earlier version of the Catalog, tracebacks removed all but two stars from Carina (see Section 7.17). Carina has a spatial volume only slightly larger than $\eta \mathrm{Cha}, \epsilon$ Cha, and Ursa Major. The most massive member of Carina is HD 83096, an F0V star (which has a lower mass than most moving groups' largest members; see also Section 7.8).

Most members of Carina have low, but non-zero, probabilities of membership in Columba or Tuc-Hor. It is also a fairly poorly recovered group by all moving group codes studied; the best recovery in the lithium sample was 8 of 21 members, by both BANYAN and LACEwING in Young Star mode.

\subsection{Carina-Vela}

Discovered by Makarov \& Urban (2000), Car-Vel was suspected of being related to the IC 2391 open cluster in much the same way as the IC 2391 Supercluster (Eggen 1991), although they share no members. Most of the stars thought to be in Car-Vel are now assigned as field stars, members of Carina, or members of Argus. This group was never considered for inclusion in LACEwING.

\subsection{Argus}

Argus was first identified by Torres et al. (2008, p. 757) as an improvement on the Car-Vel moving group, with which it shares some members. Argus shares a $U V W$ velocity with the IC 2391 open cluster and is thought to be the product of the same star-forming event (De Silva et al. 2013). This association is problematic: with the cluster at a distance of $\sim 150 \mathrm{pc}$ (Caballero \& Dinis 2008), it is not clear how the stars could have reached the vicinity of the Sun yet have space velocities parallel to IC 2391 at the present day. Argus is also problematic in that Bell et al. (2015) found the members to not be co-eval and failed to compute an age for the NYMG. The hottest member of Argus is $\epsilon \mathrm{Pav}$ (A0V).
The problematic nature of Argus may also be exhibited in the lithium sample study, where none of the codes managed to find even half of the 28 lithium-detected members of Argus.

\subsection{AB Dor}

AB Dor was first identified by Torres et al. (2003) as "AnA" and by Zuckerman et al. (2004) as AB Dor (and possibly prediscovered by Asiain et al. 1999 as subgroup B4.) AB Dor has a space velocity distribution encompassing that of the Pleiades, and has been thought to be a product of the same star-forming event (Luhman et al. 2005; Ortega et al. 2007). Though initially thought to be a younger population at $50 \mathrm{Myr}$ (Close et al. 2005), it is now believed to be as old as $150 \mathrm{Myr}$ (Bell et al. 2015), which would make it older than the Pleiades. AB Dor is the largest of the NYMGs based on the number of systems with BAFGK primaries. However, AB Dor is old enough that lithium and surface gravity are less effective indicators for low-mass objects, and chemical tagging studies (Barenfeld et al. 2013; McCarthy \& Wilhelm 2014) have determined that perhaps as many as half of the AB Dor members may be interlopers. AB Dor is effectively an all-sky moving group. The hottest member of AB Dor is Alnair (B6V).

LACEwING recovers all but eight bona fide members of $A B$ Dor in Field star mode and all but five members in young star mode; in all cases the rejected stars match no other moving group. In both cases, it failed to recover Alnair and $\delta \mathrm{Scl}$ (the hottest member that it does recover is HR 1014, A3V).

\subsection{Carina-Near}

Car-Near was identified by Zuckerman et al. (2006) as a nearby older population of stars. The hottest member of CarNear is HR 3070 (F1), which is cooler than most group's hottest members (see Sections 7.8 and 7.11).

Car-Near is not well recovered by LACEwING due to its large volume and small membership. One member (LP 356-14) is identified in both young and field star modes as a member of Argus, the group whose $U V W$ velocity Car-Near most closely resembles. HR 3070 is an Argus member in Young Star mode. The rest match either Car-Near or no group at all. Despite this poor recovery, Car-Near still appears to be a real group. It does not have an unusually large present-day volume like Oct-Near (Section 7.16) and a corresponding complete failure of recovery, its members are largely only members of the group itself, and it does produce self-consistent tracebacks, unlike Her-Lyr (Section 7.17). It seems most likely that other members of Car-Near will be found, increasing the spatial density and therefore the recovery rate.

\subsection{Octans-Near}

Oct-Near was identified by Zuckerman et al. (2013) as a potential very-nearby association of stars (including EQ Peg $\mathrm{AB}$ at $6.2 \mathrm{pc}$ ) with similar velocities to Octans and Castor. Though considered for inclusion in LACEwING, Oct-Near posed two problems for inclusion. First, as presented in Zuckerman et al. (2013), the group had multiple apparent ages between 30 and $200 \mathrm{Myr}$. Second, the present-day moving group ellipses were more than three times the size of the other moving groups and the resulting groups so sparse that LACEwING could not recover any of the supposed bona fide members. Accordingly, it is not included in this 
implementation of LACEwING. The hottest member of Octans-Near was 34 Psc (B9 V).

\subsection{Hercules-Lyra}

Her-Lyr was first identified by Gaidos (1998) and Fuhrmann (2004), and was comprised almost entirely of nearby stars; it would have been another all-sky moving group. The existence of Her-Lyr has been disputed for some time; as Mamajek (2015) notes, multiple papers (most recently, Eisenbeiss et al. 2013) have identified members of Her-Lyr, but none have consistent lists of members. Nevertheless, in recognition of the fact that the supposed members of Her-Lyr were a population of lithium-rich stars older than $\mathrm{AB}$ Dor, we attempted to retain Her-Lyr. Her-Lyr did appear in the preliminary LACEwING calibration used in Riedel (2016b) and Faherty et al. (2016), but with data from our updated Catalog of Suspected Nearby Young Stars (Section 4), only one star-V0439 And-remained within the boundaries of Her-Lyr and survived traceback filtering. Her-Lyr is not included in this implementation of LACEwING. The hottest member of Her-Lyr was $\alpha$ Cir (A7V), which was not listed as a bona fide member in Eisenbeiss et al. (2013).

\subsection{Castor}

Castor was first identified by Anosova \& Orlov (1991) in a relatively wide $\left(6 \mathrm{~km} \mathrm{~s}^{-1}\right)$ search for potential clusters around prominent triple star systems. Though refined in multiple papers over the ensuing 25 years, the existence of Castor has been questioned and debunked by Mamajek et al. (2013) on the grounds that the most prominent members (Vega, Fomalhaut, LP 944-020, and Castor) were nowhere near each other even $10 \mathrm{Myr}$ ago and could not have formed in the same molecular cloud. This determination was made with a different data set and a simple linear traceback. The existence of Castor was also disputed by Zuckerman et al. (2013) on the basis of differing ages and large velocity spreads within the group.

We have attempted to trace back Castor ourselves, starting with all 84 members ever identified as Castor members (regardless of current identification) from the Catalog of Suspected Nearby Young Stars with parallaxes and radial velocities. Only 33 stars survived three rounds of TRACEwING filtering, at which point the group included neither Castor itself nor LP 944-020, and was still the largest moving group in terms of volume at formation (Figure 17). Castor's volume at formation is indistinguishable from the size of the fake moving group of field stars. Castor, a sextuple system with four A-type stars, is likely both the hottest and most massive member of the moving group.

We cannot conclusively rule out the existence of Castor because it is still smaller than our simulated moving group between the ages of 200-400 Myr (covering both the age given by Barrado y Navascues 1998 and the ages of the prominent members collected in Mamajek et al. 2013), but we suspect this is further evidence that Castor is not a real moving group, and we have not included it in LACEwING.

\subsection{Ursa Major}

The Ursa Major moving group, often referred to as the Sirius Supercluster in older literature and occasionally referred to as a cluster (Mamajek 2015), was the first moving group discovered, by R. A. Proctor in the late 19th century. Castro et al.
(1999) published a chemical tagging analysis that revealed that the group members are identifiably rich in barium. King et al. (2003) published the most recent large-scale study of the group and determined that Sirius is not a likely member. Ursa Major is represented here by only the core members from King et al. (2003) and is thus one of the smallest moving groups in LACEwING. The hottest member of Ursa Major is $\epsilon \mathrm{UMa}$ (A0pCr), although Mizar-Alcor, a sextuplet with five A-type stars, is likely to be more massive.

Ursa Major is another troubling group; the final traceback set of Ursa Major members did not include Mizar-Alcor, and the resulting LACEwING calibration missed 8 of the 11 lithiumrich members in young mode (although LACEwING does recover Mizar-Alcor as a member).

\subsection{Coma Berenices Open Cluster}

Coma Ber (Melotte 111, Collinder 256) is an open cluster 86 pc distant consisting of roughly 195 stars from our limited literature search, of which 104 are BAFGK members. Thus, Coma Ber is second only to the Hyades in terms of size. Coma Ber was a difficult moving group to add to our simulation of the Solar Neighborhood because it has a very low $U V W$ velocity and the simulation produced proper motions indistinguishable from zero for many of the simulated members. Fortunately, LACEwING's field population prevents LACEwING from identifying large portions of the sky as Coma Ber members. The hottest member of Coma Ber is AI Com (A0p).

\subsection{1. $\chi^{01}$ Fornax}

$\chi^{01}$ For (also known as Alessi 13) was first published in a catalog by Dias et al. (2002). It has remained obscure for the past decade, but appears to be real (E. E. Mamajek 2016, private communication). The only available age estimates are from Pöhnl \& Paunzen (2010) and Kharchenko et al. (2013), which hover around $500 \mathrm{Myr}$, though Mamajek believes the group may be younger (Mamajek 2016). For the purposes of traceback we intended to use $450-550 \mathrm{Myr}$, but only three members of the group have parallaxes. We used the $U V W$ properties from the private communication with E. E. Mamajek instead, and calculated the $X Y Z$ positions from the $\alpha, \delta$, Dist., and tidal radius. The hottest member of $\chi^{01}$ For is $\chi^{01}$ For (A1V) itself.

\subsection{Hyades Open Cluster}

The Hyades open cluster (Melotte 25, Collinder 50) has been known since antiquity. Based on analyses of Röser et al. (2011) and Goldman et al. (2013), there are 724 known members of the Hyades, of which 260 are BAFGK members (including giants and white dwarfs). The hottest stellar member (excluding white dwarfs) is $\theta^{02}$ Tau, an A7III giant. The age is believed to be between 600 (Zuckerman \& Song 2004) and $800 \mathrm{Myr}$ (Brandt \& Huang 2015), and is the upper limit of what we consider to be a young group.

The Hyades is represented here by a conversion of the properties in van Leeuwen (2009) to $U V W X Y Z$.

\subsection{Young Nonmembers}

A large fraction of our lithium sample-582 of the 930 star systems - do not trace back to any of the NYMGs in young star mode. Twenty-four of these just miss $(>10 \%$ probability of 
membership in at least one group) the usual $20 \%$ probability cut, which could be due to only having proper motion available for membership assignment, but that still leaves 534 of 930 systems $(57 \%)$ as nonmembers of the groups. This behavior is not unique to LACEwING. Running the same sample through BANYAN, which had the highest recovery rate of any of the codes, 589 stars fell into the "Old" category. BANYAN II in field star mode identified 369 stars as young field objects and 355 stars as old field objects; in young star mode it identified 720 members of the lithium sample as "Old." This is not a perfect comparison, as the BANYAN codes test for fewer groups than LACEwING, and some of the lithium-detected stars are members of groups older than BANYAN's oldest.

If we break down the entire lithium sample, only 487 of the star systems have ever been considered as potential members of groups that LACEwING tests for. An additional 79 have only been considered as members of moving groups LACEwING does not test for (Her-Lyr, Oct-Near, Castor, Car-Vel, Local Association, Hyades Supercluster, IC 2391 Supercluster). The remaining 364 systems have never been considered as members of any specific young group.

This general behavior has been noted before, particularly in surveys that did not filter by kinematic match before following up stars for further observations (Shkolnik et al. 2009, 2012; Riedel et al. 2014, 2016b; Binks et al. 2015). These results have suggested that there is an unidentifiable population of young stars.

If we accept that these stars are young, there are five possibilities for their origins.

1. The stars did form as part of the known NYMGs, and flaws in our data or models are responsible for the stars that are not associated with a group.

2. The stars did form as part of the known NYMGs, but dynamical interactions (most likely an ejection from a higher-order multiple star system early in its evolution) gave them high relative velocities such that they do not kinematically match the group that they formed with. Chemical tagging would be useful for identifying such systems, provided it is possible to uniquely identify a moving group in that way. Kinematic tracebacks that place a star near another member of a moving group of the appropriate age would be suggestive, but would require advancements in both data precision and technique from what is presented here.

3. The stars did form as part of groups that are known but not nearby. LACEwING only tests for groups currently known to extend within $100 \mathrm{pc}$ of the Sun. The ScorpiusCentaurus and Taurus-Auriga star-forming regions are less than $200 \mathrm{pc}$ away. With a typical velocity dispersion of $1.5 \mathrm{~km} \mathrm{~s}^{-1}$ (Preibisch \& Mamajek 2008), a star could move from $118 \mathrm{pc}$ (the canonical distance to the $\sim 16 \mathrm{Myr}$ old Lower Centaurus Crux region of Scorpius-Centaurus; Preibisch \& Mamajek 2008) to within 100 pc of the Sun in just over $10 \mathrm{Myr}$.

4. The stars did form as part of unknown NYMGs that we have not yet discovered. 32 Ori and $\chi^{01}$ For are relatively unexplored groups; the All Sky Young Association (Torres et al. 2016) has been announced but no particulars have yet been given. Other, smaller groups that have no members more massive than $\mathrm{M}$ dwarfs may yet be hiding in the Solar Neighborhood.
5. The stars did not form as part of any groups at all, and were instead part of a one-off star formation event. The NYMGs already range greatly in size from the $\eta$ Cha open cluster (21 known systems as of 2015 January) to the Tuc-Hor moving group (209 known systems as of 2015 January); it is not clear what the smallest star formation event can be.

\section{Conclusions}

We have introduced the LACEwING moving group identification code, which uses kinematics to determine the probability of membership in 13 NYMGs and 3 open clusters within $100 \mathrm{pc}$. We have introduced the TRACEwING epicyclic traceback code, which uses an epicyclic approximation to Galactic orbital motion to trace stars back to their origins. We have also introduced the catalog of suspected young stars, which contains a wide variety of kinematic, spectroscopic, photometric, and membership information on 5350 nearby stars that have been identified in the literature as potentially young.

We have demonstrated that LACEwING produces reliable results consistent with current expectations, for the first time across all known moving groups within 100 pc. Despite handling a large number of moving groups, LACEwING's recovery rates are in line with other previously established moving group codes like BANYAN and BANYAN II. By including more moving groups in the kinematic identification, we make it substantially easier to identify members and obtain ages for nearby stars. Uniform and repeatable determinations are now possible for groups with a wide range of ages, covering a wide range of youthful states.

The TRACEwING epicyclic traceback code allows us to identify objects based on their spatial origins, which should be a more fundamental constraint than present-day kinematics, particularly once higher precision data is available. On the issue of populations, it should provide a useful means for testing the spatial formation scenarios of the NYMGs.

The Catalog of Suspected Nearby Young Stars constitutes a valuable resource for studying the large-scale properties of nearby young stars in both an individual and populational basis. It will be maintained as part of a larger database of young stars (Hillenbrand \& Baliber 2015, currently under construction), and made available to other researchers for use as a source of data for a wide variety of studies.

The Gaia mission, due to its precision, accuracy, and extraordinary magnitude range, will make it possible to perform analyses like this on stars beyond 100 pc from the Sun. Gaia data will allow us to conclusively answer the question of the existence of groups like Castor. Gaia should make it possible to test the various theories to explain the origins of the young field, and potentially break up the currently known groups into smaller, more physically meaningful groups just as the Hipparcos mission did for the previously known stellar streams.

Based on the analysis done here, the most certain groups are $\epsilon$ Cha, $\eta$ Cha, TW Hya, $\beta$ Pic, 32 Ori, Tuc-Hor, AB Dor, Coma Ber, and $\chi^{01}$ For; the existence of the Hyades is also not in doubt. More work needs to be done on the rest of these groups. That includes the Columba and Carina moving groups in particular, which may be part of Tuc-Hor; the 32 Ori (and $\chi^{01}$ For) groups that have not been well studied to date; and groups 
like Octans, Argus, Car-Near, and Oct-Near that appear to be problematic in size or recovery performance.

A.R.R. acknowledges support from NSF grant AST-131278, NASA ADAP grant NNX12AD97G, and generous support from the Office of the Provost at the College of Staten Island, City University of New York. The authors wish to thank J. Gagné, L. Malo, D. R. Rodriguez, and E. E. Mamajek for helpful suggestions and commentary, G. Schwarz for help with catalog preparation, J. L. McDonald for editorial assistance, and our two referees, J. A. Caballero and an anonymous referee, whose suggestions greatly improved the quality and readability of the paper, the code, and the data products.

This publication makes use of data products from the Two Micron All Sky Survey, which is a joint project of the University of Massachusetts and the Infrared Processing and Analysis Center/California Institute of Technology, funded by the National Aeronautics and Space Administration and the National Science Foundation.

This publication makes use of data products from the Widefield Infrared Survey Explorer, which is a joint project of the University of California, Los Angeles, and the Jet Propulsion Laboratory/California Institute of Technology, funded by the National Aeronautics and Space Administration.

This research has made use of the Washington Double Star Catalog maintained at the U.S. Naval Observatory. This research was also made possible through the use of the AAVSO Photometric All-Sky Survey (APASS), funded by the Robert Martin Ayers Sciences Fund. This research has made extensive use of the SIMBAD database and VizieR catalog access tool operated at CDS, Strasbourg, France. The original description of the VizieR service was published in A\&AS $143,23$.

Software: Astropy (Astropy Collaboration et al. 2013), Numpy (Walt et al. 2011), Scipy (Jones et al. 2001), Matplotlib (Hunter 2007), LACEwING (Riedel 2016a)

\section{Appendix A LACEwING Manual}

LACEwING is available from GitHub ${ }^{14}$ (as of 2016 December 5) and is indexed in the Astronomy Source Code Library (Riedel 2016c). ${ }^{15}$ The version used in this article is archived in Zenodo (Riedel 2016a).

To run LACEwING to obtain membership probabilities, the following are required:

1. A Python 2.7 interpreter with numpy, astropy, and matplotlib.

2. lacewing.py - the main routines necessary for LACEwING, specifically.

3. kinematics.py-routines to convert between equatorial $(\alpha, \delta, \pi)$ and Galactic $(X Y Z)$ coordinates (and also kinematic tracebacks).

4. ellipse.py-ellipse fitting and rotation routines.

5. astrometry.py-proper motion conversion routines and other important miscellaneous routines.

6. Moving_Groups_all.csv-A comma-separated value format file with the precalculated moving group parameters

\footnotetext{
${ }^{14}$ https://github.com/ariedel

15 http://ascl.net/1601.011
}

7. The files on GitHub contain an additional file, Moving_Groups_all_prelim.csv, which contains the preliminary moving groups used by Faherty et al. (2016). Rename to Moving_Groups_all.csv to use.

The gal_uvwxyz function in kinematics.py is a modified version of gal_uvw originally written by Wayne Landsman for the IDL Astronomy User's Library (under a two-clause BSD license) and converted to Python 2 by Sergey Koposov.

\section{A.1. General Usage}

The LACEwING algorithm is available as a function that can be called from other programs (see Appendix A.2), but if it is run directly from the command line it defaults to reading from a comma-separated value file:

$$
\begin{aligned}
& \text { python lacewing.py inputfile [calibration] } \\
& \text { [output filename] [verbose output] [g.o.f] }
\end{aligned}
$$

LACEwING (using the astropy.io.ascii module) looks for a one-line header with any or all of the columns selected below (broadly, they are either common names for the quantities or AAS Journal standards). These can be given in any order; any other headers present in the file are ignored. Most of the errors are optional and if they (and their header) are not present, LACEwING will substitute default values.

1. Name-ascii, any length; should not contain commas itself.

2. RA, DEC (or RAdeg, DEdeg)—right ascension and declination in decimal degrees, J2000/ICRS equinox.

3. RAh, RAm, RAs, DE-, DEd, DEm, DEs-sexagesimal coordinates, J2000/ICRS equinox, split into seven columns with a separate declination $+/-$ flag. These will only be read if RA and DEC are invalid, empty, or do not exist.

4. eRA, eDEC (or e_RAdeg, e_DEdeg)—R.A.cosdecl. and decl. uncertainties in milliarcseconds. These, and their header keyword, are optional and will default to 1000 milliarcseconds.

5. pmRA, pmDEC (or pmRA, pmDE; or pmra and pmdec) $-\mu_{\text {R.A. cos decl. }}$ and $\mu_{\text {decl. }}$ in mas $\mathrm{yr}^{-1}, \quad$ J2000/ICRS equinox.

6. epmRA, epmDEC (or e_pmRA,e_pmDE; or epmra, epmdec) $-e \mu_{\text {R.A. cos decl. and } e \mu_{\text {decl. }} \text { uncertainties }}$ in mas $\mathrm{yr}^{-1}$, J2000/ICRS equinox. These, and their

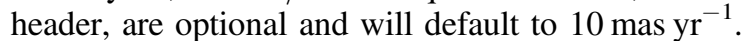

7. pi (or plx) - trigonometric parallax in milliarcseconds. It is highly recommended that the photometric parallax estimates are not used.

8. epi (or e_plx) - trigonometric parallax uncertainty in milliarcseconds. This must be present in order to use the parallax.

9. rv (or HRV) —radial velocity in $\mathrm{km} \mathrm{s}^{-1}$.

10. erv (or e_HRV)—radial velocity uncertainty in $\mathrm{km} \mathrm{s}^{-1}$. This must be present in order to use the radial velocity.

11. Note-any text you wish to have duplicated in the file output, such as a previously known membership assignment.

The additional command-line parameters are

1. calibration-type "young" to switch to the alternative LACEwING calibration where stars are assumed to be young. Defaults to "field" 
2. output filename-enter an output filename, otherwise LACEwING defaults to lacewing_output.csv.

3. verbose-If this is "verbose," LACEwING will output a more detailed report as described below. It defaults to a much more compact output format

4. g.o.f-If this is set to anything other than "percentage," LACEwING outputs the combined goodness-of-fit statistic rather than determining the moving group membership probability.

The LACEwING output format, in the regular version, is a comma-separated value file containing, in order:

1. Star name

2. Note (directly from the input)

3 . Best-matching moving group

4. Membership probability for the best-matching moving group (unless the "g.o.f" flag is set on the command line)

5. Kinematic distance (in parsecs) for the best-matching group

6. Kinematic distance uncertainty (in parsecs) for the bestmatching group

7. Kinematic radial velocity (in $\mathrm{km} \mathrm{s}^{-1}$ ) for the bestmatching group

8. Kinematic radial velocity uncertainty (in $\mathrm{km} \mathrm{s}^{-1}$ ) for the best-matching group

9. Membership probability for $\epsilon$ Cha

10. Membership probability for $\eta$ Cha

11. Membership probability for TW Hya

12. Membership probability for $\beta$ Pic

13. Membership probability for 32 Ori

14. Membership probability for Octans

15. Membership probability for Tuc-Hor

16. Membership probability for Columba

17. Membership probability for Carina

18. Membership probability for Argus

19. Membership probability for AB Dor

20. Membership probability for Carina-Near

21. Membership probability for Coma Ber

22. Membership probability for Ursa Major

23. Membership probability for $\chi^{01}$ For

24. Membership probability for Hyades

The Verbose Output formula is drastically different, consisting of a block of 16 lines detailing the match between the star and every group, one at a time. It is most useful for tracking down the reason why a particular star did not match a particular group.

1. Name

2. R.A. (degrees, from the input)

3. Decl. (degrees, from the input)

4. Moving group

5. Text string: "PROB $=$ " (or "SIG=," if the g.o.f. flag has been set on the command line)

6. Membership probability in the moving group (or combined goodness of fit if the g.o.f. flag has been set)

7. Text string: "PM="

8. Proper motion goodness-of-fit metric

9. Kinematic (predicted) $\mu_{\text {R.A. cos decl. }}\left(\right.$ mas yr $\left.^{-1}\right)$

10. Kinematic $\mu_{\text {R.A. cos decl. uncertainty (mas } \mathrm{yr}^{-1} \text { ) }}$

11. Kinematic $\mu_{\text {decl. }}\left(\right.$ mas $\left.\mathrm{yr}^{-1}\right)$

12. Kinematic $\mu_{\text {decl. }}$ uncertainty (mas $\mathrm{yr}^{-1}$ )

13. Measured $\mu_{\text {R.A. cos decl. }}\left(\right.$ mas $\left.\mathrm{yr}^{-1}\right)$
14. Measured $\mu_{\text {R.A. cos decl. }}$ uncertainty ( ${\operatorname{mas~} \mathrm{yr}^{-1} \text { ) }}^{-1}$

15. Measured $\mu_{\text {decl. }}\left(\right.$ mas $\left.\mathrm{yr}^{-1}\right)$

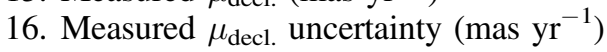

17. Text string: "DIST $="$

18. Distance goodness-of-fit metric

19. Kinematic distance (parsecs)

20. Kinematic distance uncertainty (parsecs)

21. Measured distance $\left(\frac{1}{\pi}\right.$, parsecs)

22. Measured distance uncertainty $\left(\frac{\sigma_{\pi}}{\pi^{2}}\right.$, parsecs $)$

23. Text string: " $\mathrm{RV}=$ "

24. Radial velocity goodness-of-fit metric $\left(\mathrm{km} \mathrm{s}^{-1}\right)$

25 . Kinematic radial velocity $\left(\mathrm{km} \mathrm{s}^{-1}\right)$

26. Kinematic radial velocity uncertainty $\left(\mathrm{km} \mathrm{s}^{-1}\right)$

27. Measured radial velocity $\left(\mathrm{km} \mathrm{s}^{-1}\right)$

28. Measured radial velocity uncertainty $\left(\mathrm{km} \mathrm{s}^{-1}\right)$

29. Text string: "POS=" (or "KPOS=," if the spatial position is based on the kinematic distance)

30. Spatial position goodness-of-fit metric

31. 3D separation between star and center of moving group (parsecs)

32. 3D separation uncertainty (parsecs)

33. Note (directly from the input)

The lacewing_summary.py function will summarize the verbose output into the regular format.

python lacewing_summary.py inputfile

The output file will be named inputfile.summary.csv

\section{A.2. LACEwING As a Function}

LACEwING as a function requires two calls. One, lacewing. moving_group_loader() returns a list of moving group classes loaded from the Moving_Group_all.csv file. This must be done before lacewing.lacewing(), so the resulting list can be passed to lacewing.lacewing(). This is done to avoid the redundancy of reloading the parameters every time.

lacewing.lacewing () accepts data on a single object at a time, and cannot be fed lists, arrays, or tuples. The returned list of moving group classes must be passed to lacewing.lacewing() every time. The first argument is required:

1. moving_group-list, moving group class list (as created by lacewing.moving_group_loader()).

The remainder of the items are optional; if not present (or explicitly set to NoneType None) lacewing() will treat them as unknowns.

2. young $=$ string, if this is "young" then lacewing() will use the young star calibration. Otherwise (or if None, or not specified), lacewing() will use the field star calibration

3. $\mathrm{ra}=$ float, decimal degrees, J2000/ICRS coordinates

4. era $=$ float, R.A. cos decl. uncertainty in decimal degrees

5. $\operatorname{dec}=$ float, decimal degrees, J2000/ICRS coordinates

6. edec $=$ float, decl. uncertainty in decimal degrees

7. pmra $=$ float, $\mu_{\text {R.A. cos decl. }}$ in $\operatorname{arcsec} \mathrm{yr}^{-1}$

8. epmra $=$ float, $\mu_{\text {R.A. cos decl. }}$ uncertainty in $\operatorname{arcsec} \mathrm{yr}^{-1}$

9. $\mathrm{pmdec}=$ float, $\mu_{\text {decl. }}$ in $\operatorname{arcsec} \mathrm{yr}^{-1}$

10. epmdec $=$ float, $\mu_{\text {decl. }}$ uncertainty in $\operatorname{arcsec} \mathrm{yr}^{-1}$

11. $\mathrm{plx}=$ float, $\pi$ in arcseconds

12. eplx $=$ float, $\pi$ uncertainty in arcseconds

13. $\mathrm{rV}=$ float, $\mathrm{RV}$ in $\mathrm{km} \mathrm{s}^{-1}$

14. erv $=$ float, $\mathrm{RV}$ uncertainty in $\mathrm{km} \mathrm{s}^{-1}$ 
Note that lacewing() does not check to see if the uncertainties exist (or are not None), so (for example) supplying rv without erv will cause lacewing() to crash with a TypeError.

The output from lacewing() is a list of dicts, one dict per moving group in the order they appear in Moving_Group_all. csv. Each dict contains the following keys (which will be None unless data exists):

1. "group"-string, group name

2. "gof"-float, goodness-of-fit parameter

3. "probability" float, membership probability (percent)

4. "pmsig"-float, proper motion match metric

5. "kin_pmra"-_float, estimated $\mu_{\text {R.A. } \cos \text { decl. }}\left(\operatorname{arcsec} \mathrm{yr}^{-1}\right)$

6. "kin_epmra"-float, estimated $\mu_{\text {R.A. cos decl. uncertainty }}$ $\left(\operatorname{arcsec} \mathrm{yr}^{-1}\right)$

7. "kin_pmdec"-float, estimated $\mu_{\text {decl. }}\left(\operatorname{arcsec} \mathrm{yr}^{-1}\right)$

8. "kin_epmdec"-float, estimated $\mu_{\text {decl. }}$ uncertainty $\left(\operatorname{arcsec} \mathrm{yr}^{-1}\right)$

9. "distsig"-float, distance match metric

10. "kin_dist"-float, expected distance (parsecs)

11. "kin_edist"-float, expected distance uncertainty (parsecs)

12. "rvsig"- float, radial velocity match metric

13. "kin_rv"_-float, expected radial velocity $\left(\mathrm{km} \mathrm{s}^{-1}\right)$

14. "kin_erv"_float, expected radial velocity uncertainty $\left(\mathrm{km} \mathrm{s}^{-1}\right)$

15. "possig"-float, position match metric (using measured distance)

16. "pos_esig"_-float, position match metric uncertainty

17. "pos_sep"-float, distance from moving group center (parsecs)

18. "posksig"-float, position match metric (using kinematic distance)

19. "pos_eksig"_float, kinematic position match metric uncertainty

20. "pos_ksep"-float, kinematic distance from moving group center (parsecs)

There are two example implementations of lacewing. lacewing() in the repository: The default reader within the lacewing.py file, and the sample star generator in lacewing_montecarlo.py.

\section{A.3. Adding a Moving Group to LACEwING}

Step 1: Add the group to Moving_Groups_all.csv.

Generate new moving group parameters-Create a commaseparated value file in the format described above for the default csv loader, containing data on every member of the group.

Run this file through the moving group maker:

python lacewing_mgpmaker.py inputfile

Also, run this through the $2 \mathrm{D}$ version to get parameters for lacewing_uvwxyz.py

python lacewing_mgpmaker2d.py inputfile

The output files are .csv files named "Moving_Group_Group Name.dat" and "Moving_Group_Group Name_2d.dat" with $U$, $V, W, A, B, C, U V, U W, V W, X, Y, Z, D, E, F, X Y, X Z$, and $Y Z$ values, with uncertainties (which LACEwING ignores) for each value on the second line. The first line is suitable for entering into Moving_Groups_all.csv. The 2D versions of those values should be stored in the A2, B2, C2, UV2 (etc.) columns. Note that $U V W$ and $X Y Z$ values are the same for $2 \mathrm{D}$ and $3 \mathrm{D}$ projections, so there are no special $2 \mathrm{D}$ entries for them.

Alternatively, if you have the more customary $U V W$ and $X Y Z$ values (no rotations) from a different source, you can enter those into the file, specifying 0 for all the rotation angles. The same values will apply for $3 \mathrm{D}$ and $2 \mathrm{D}$.

Column "Name" should contain the name of the group, preferably less than 20 characters, with underscores instead of spaces.

Column "Number" is a best estimate of the current known members of the group.

Column "Weightednumber" is a cumulative fraction of stars in each group,

$$
\frac{\sum_{0}^{i} \text { Number }_{\text {group (i) }}}{\sum_{0}^{N} \text { Number }_{\text {group (i) }}},
$$

such that all are between 0 and 1, and should be generated from the number of members.

Column "uniform" specifies whether the group should be simulated as (0) a uniform spatial and Gaussian velocity distribution (unused), (1) a Gaussian spatial and Gaussian velocity distribution, like an open cluster, or (2) a spatial distribution with a scale height of $300 \mathrm{pc}$ and a Gaussian velocity distribution, like the field population.

You can also add an Age (unused), References (unused), and the fractional RGB color components (for plots, such as lacewing_uvwxyz.py). Also, fill the membership coefficients section with zeros so that the program will function initially.

If you are removing a group, just delete its line and recompute the "Weightednumber" column.

Step 2: Generate a new simulation-Generate a new Monte Carlo simulation.

\section{python lacewing_montecarlo.py iterations number}

where "iterations" is the number of stars to draw out of the distributions now listed in Moving_Groups_all.csv. "Number" is a value to append to the names of the output files so that they will be kept separate, and is a way of more efficiently using computer resources.

Good values for "number" are the number of cores you are willing to devote to this process. Good values for "iterations" depends on the number of members in the least-populated group. This process needs roughly 1000 simulated members in total to populate the eventual membership histograms well enough to fit them. So, if you have a quad-core machine and the least-populated group accounts for fractionally 0.0001 of the stars, you need 10 million points and should generate 5 million with four running instances. An Intel Core i7 4700MQ running eight instances can generate about two million stars per day, and 8,000,000 entries takes up roughly $610 \mathrm{MiB}$ of space per moving group.

The program will use random number generators to follow the procedure in Section 2.2 and generate simulated stars to run through lacewing.lacewing().

The output will be number files for each moving group, each containing iterations entries, with the goodness-of-fit values matching each star to the moving group using all seven possible combinations of input data, and a record of what group the star was generated as a member of. 
Concatenate the files for each group into one file per group. It is preferable to move them to another subfolder.

Step 3: Generate and fit probability histograms-Run lacewing_percentages.py on the Monte Carlo output.

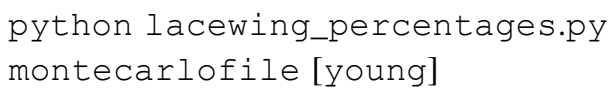

This program generates the histogram of "percentage of objects at each goodness-of-fit value that are actual members." Run it on one of the files from the Monte Carlo output to generate a wide variety of plots (as appearing in Section 2.2) and a file called group.percentages. This file contains the cumulative Gaussian fit parameters that need to be saved in Moving_Groups_all.csv. All of these files are stored in a folder called montecarlo, which is created if it does not exist.

If you specify "young," the program will generate the young star calibration; as it combs through the input file it will ignore all but an equal number of field stars to provide the case where the stars are assumed to be young. These calibrations (in group. young.percentages) should also be saved in Moving_Groups_all.csv, replacing the zeroes put there earlier.

You can now use LACEwING to predict members of your new moving group.

\section{A.4. lacewing_uvwxyz.py}

Requirements:

1. A Python 2.7 interpreter with numpy, astropy, and matplotlib

2. lacewing.py - the main routines necessary for LACEwING, specifically the csv loader

3. lacewing_uvwxyz.py-the UVWXYZ fitting and plotting tool

4. kinematics.py-routines to convert between equatorial (R.A., decl., $\pi$ ) and Galactic (XYZ) coordinates (and also kinematic tracebacks)

5. ellipse.py-ellipse fitting and rotation routines

6. astrometry.py-proper motion conversion routines and other important miscellaneous routines

7. Moving_Groups_all.csv-a comma-separated value format file with the precalculated moving group parameters

\section{A.4.1. General Usage}

lacewing_uvwxyz.py calculates $X Y Z$ (where parallaxes exist) and $U V W X Y Z$ (where all six elements of kinematics exist) values, which are output to an output filename. It also generates $2 \mathrm{D}$ projection plots of stars plotted on the $U V W$ and $X Y Z$ spatial axes, as shown in Figure 18:

python lacewing_uvwxyz.py inputfile [output

filename] [XYZ]

Input file format is the same as lacewing; it uses the same . csv loader).

The next two arguments are optional:

1. output filename-enter an output filename, otherwise LACEwING defaults to lacewing_output.csv

2. $X Y Z$ - if this string is " $X Y Z$," the output .png image will have a second row of projected $X Y Z$ positions

There are two outputs:
1. One image per star (named starname_decimal coordinates.png) with panels showing the $U$ versus $V, U$ versus $W$, and $V$ versus $W$ projections of 3D velocity (and, if “ $X Y Z$ ' was specified, $X$ versus $Y, X$ versus $Z$, and $Y$ versus $Z$ projections of $3 \mathrm{D}$ space) similar to Figure 18

2. One output comma-separated value file with rows of Name, U, eU, V, eV, W, eW, X, eX, Y, eY, Z, and eZ values for each star.

\section{A.5. TRACEWING}

1. A Python 2.7 interpreter with numpy, astropy, and matplotlib

2. tracewing.py - the kinematic traceback tool

3. lacewing.py-for the csv loader

4. kinematics.py-routines to trace the star back in time with epicyclic tracebacks

5. ellipse.py-ellipse fitting and rotation routines

6. astrometry.py-proper motion conversion routines and other important miscellaneous routines

7. Moving_Group_Group Name_epicyclic.dat-stored parameter files for the moving groups. The files on GitHub have been calculated back to $-800 \mathrm{Myr}$.

tracewing.py computes the traceback of stars to a given moving group, which must be present in a saved parameter file. The outputs are .png figures of the star traced back to the NYMG (Figure 7), covering the time between 0 and the end of the plotting time period.

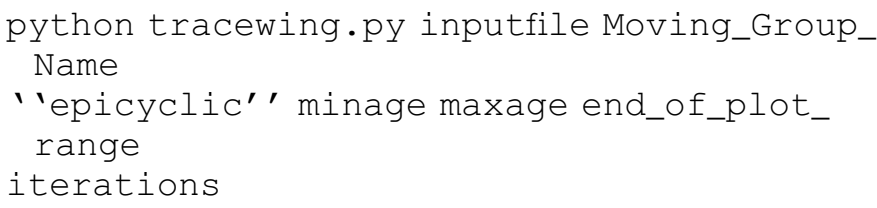

Inputfile should be in the same file format as LACEwING. The Moving Group Name should be "beta_Pic" if the name is "Moving_Group_beta_Pic_epicyclic.dat." "epicyclic" is the only method tested and with moving group data to trace back to. The ages and end of plot range must all be negative, but greater than $-800 \mathrm{Myr}$. The number of iterations should determine the quality of the plot; 10,000 is likely more than enough.

\section{A.6. Generating New Moving Groups}

To create a new NYMG from a group of stars, assemble all the stellar properties of the NYMG in a file inputfile (same format as lacewing.py and tracewing.py). Then run the following:

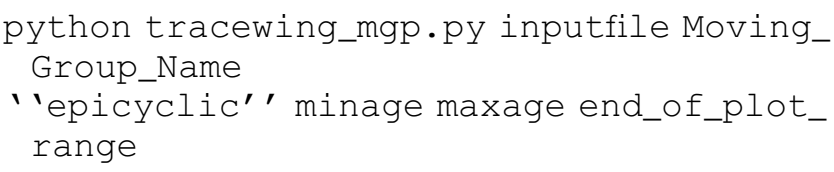

Tracewing_mgp.py has all the same requirements as tracewing.py. This will trace back all the stars in the file to $-800 \mathrm{Myr}$, fit ellipses at every $0.1 \mathrm{Myr}$ step, and save the output to a file Moving_Group_Group Name_epicyclic.dat suitable for use in tracewing.py. It will also attempt to generate 
a .png figure of all the stellar positions from 0 to the end of the plot range, with a blue bar drawn in over the min and max age of the group, as shown in Figure 14.

Be aware that this will require several gigabytes of memory, as it must hold $N_{\text {members }} \times 1000 \times 8000$ positions in memory.

\section{Appendix B \\ Contents of the Catalog of Suspected Nearby Young Stars}

The Catalog of Suspected Young Stars is also be available on GitHub at https://github.com/ariedel/young_catalog in its present form, and will be incorporated into the Hillenbrand \& Baliber (2015) database.

\section{References}

Ahn, C. P., Alexandroff, R., Allende Prieto, C., et al. 2012, ApJS, 203, 21 Allen, P. R., Burgasser, A. J., Faherty, J. K., \& Kirkpatrick, J. D. 2012, AJ, 144,62

Alonso-Floriano, F. J., Caballero, J. A., Cortés-Contreras, M., Solano, E., \& Montes, D. 2015, A\&A, 583, A85

Andersen, J., Clausen, J. V., Nordstrom, B., Tomkin, J., \& Mayor, M. 1991, A\&A, 246, 99

Anderson, E., \& Francis, C. 2012, AstL, 38, 331

Anosova, J. P., \& Orlov, V. V. 1991, A\&A, 252, 123

Asiain, R., Figueras, F., \& Torra, J. 1999, A\&A, 350, 434

Astropy Collaboration, Robitaille, T. P., Tollerud, E. J., et al. 2013, A\&A, 558, A33

Bailey, J. I., III, White, R. J., Blake, C. H., et al. 2012, ApJ, 749, 16

Baines, E. K., White, R. J., Huber, D., et al. 2012, ApJ, 761, 57

Barbier-Brossat, M., \& Figon, P. 2000, A\&AS, 142, 217

Barenfeld, S. A., Bubar, E. J., Mamajek, E. E., \& Young, P. A. 2013, ApJ, 766, 6

Barrado y Navascues, D. 1998, A\&A, 339, 831

Barrado y Navascués, D., Stauffer, J. R., \& Patten, B. M. 1999a, ApJL, 522, L53

Barrado y Navascués, D., Stauffer, J. R., Song, I., \& Caillault, J. 1999b, ApJL, 520, L123

Bell, C. P. M., Mamajek, E. E., \& Naylor, T. 2015, MNRAS, 454, 593

Benedict, G. F., McArthur, B., Chappell, D. W., et al. 1999, AJ, 118, 1086

Benedict, G. F., McArthur, B. E., Franz, O. G., Wasserman, L. H., \& Henry, T. J. 2000, AJ, 120, 1106

Benedict, G. F., McArthur, B. E., Gatewood, G., et al. 2006, AJ, 132, 2206

Bergfors, C., Brandner, W., Janson, M., et al. 2010, A\&A, 520, A54

Bianchi, L., Herald, J., Efremova, B., et al. 2011, Ap\&SS, 335, 161

Biller, B. A., \& Close, L. M. 2007, ApJL, 669, L41

Binks, A. S., \& Jeffries, R. D. 2014, MNRAS, 438, L11

Binks, A. S., Jeffries, R. D., \& Maxted, P. F. L. 2015, MNRAS, 452, 173

Blake, C. H., Charbonneau, D., \& White, R. J. 2010, ApJ, 723, 684

Bobylev, V. V. 2010, AstL, 36, 220

Bochanski, J. J., Hawley, S. L., Covey, K. R., et al. 2010, AJ, 139, 2679

Bouy, H., \& Alves, J. 2015, A\&A, 584, A26

Bowler, B. P., Liu, M. C., Shkolnik, E. L., et al. 2012a, ApJ, 753, 142

Bowler, B. P., Liu, M. C., Shkolnik, E. L., \& Dupuy, T. J. 2013, ApJ, 774, 55

Bowler, B. P., Liu, M. C., Shkolnik, E. L., \& Tamura, M. 2012b, ApJ, 756, 69

Brandt, T. D., \& Huang, C. X. 2015, ApJ, 807, 58

Caballero, J. A. 2009, A\&A, 507, 251

Caballero, J. A. 2010, A\&A, 514, A98

Caballero, J. A., \& Dinis, L. 2008, AN, 329, 801

Cannon, A. J., \& Pickering, E. C. 1993, yCat, 3135

Casewell, S. L., Jameson, R. F., \& Dobbie, P. D. 2006, MNRAS, 365, 447

Casewell, S. L., Littlefair, S. P., Burleigh, M. R., \& Roy, M. 2014, MNRAS, 441, 2644

Castro, S., Porto de Mello, G. F., \& da Silva, L. 1999, MNRAS, 305, 693

Close, L. M., Lenzen, R., Guirado, J. C., et al. 2005, Natur, 433, 286

Close, L. M., Thatte, N., Nielsen, E. L., et al. 2007, ApJ, 665, 736

Costa, E., Méndez, R. A., Jao, W.-C., et al. 2005, AJ, 130, 337

Covino, E., Alcala, J. M., Allain, S., et al. 1997, A\&A, 328, 187

Cutri, R. M., Skrutskie, M. F., van Dyk, S., et al. 2003, 2MASS All Sky Catalog of point sources. (NASA/IPAC Infrared Science Archive. http:// irsa.ipac.caltech.edu/applications/Gator/)
Cutri, R. M., Wright, E. L., Conrow, T., et al. 2012, Explanatory Supplement to the WISE All-Sky Data Release Products, Tech. Rep., 1C

Cutri, R. M., Wright, E. L., Conrow, T., et al. 2013, Explanatory Supplement to the AllWISE Data Release Products, Tech. Rep., 1C

Daemgen, S., Siegler, N., Reid, I. N., \& Close, L. M. 2007, ApJ, 654, 558

Dahn, C. C., Harris, H. C., Vrba, F. J., et al. 2002, AJ, 124, 1170

da Silva, L., Torres, C. A. O., de La Reza, R., et al. 2009, A\&A, 508, 833

David, T. J., \& Hillenbrand, L. A. 2015, ApJ, 804, 146

de la Reza, R., Torres, C. A. O., Quast, G., Castilho, B. V., \& Vieira, G. L. 1989, ApJL, 343, L61

Delorme, P., Gagné, J., Girard, J. H., et al. 2013, A\&A, 553, L5

De Silva, G. M., D'Orazi, V., Melo, C., et al. 2013, MNRAS, 431, 1005

Dias, W. S., Alessi, B. S., Moitinho, A., \& Lépine, J. R. D. 2002, A\&A, 389,871

Dieterich, S. B., Henry, T. J., Jao, W.-C., et al. 2014, AJ, 147, 94

Dittmann, J. A., Irwin, J. M., Charbonneau, D., \& Berta-Thompson, Z. K. 2014, ApJ, 784, 156

D’Orazi, V., Biazzo, K., Desidera, S., et al. 2012, MNRAS, 423, 2789

Ducati, J. R., Bevilacqua, C. M., Rembold, S. B., \& Ribeiro, D. 2001, ApJ, 558, 309

Ducourant, C., Teixeira, R., Chauvin, G., et al. 2008, A\&A, 477, L1

Ducourant, C., Teixeira, R., Galli, P. A. B., et al. 2014, A\&A, 563, A121

Dupuy, T. J., \& Liu, M. C. 2012, ApJS, 201, 19

Eggen, O. J. 1985, AJ, 90, 74

Eggen, O. J. 1991, AJ, 102, 2028

Eggen, O. J. 1995, AJ, 110, 2862

Eisenbeiss, T., Ammler-von Eiff, M., Roell, T., et al. 2013, A\&A, 556, A53

Elliott, P., Huélamo, N., Bouy, H., et al. 2015, A\&A, 580, A88

Faherty, J. K., Burgasser, A. J., Walter, F. M., et al. 2012, ApJ, 752, 56

Faherty, J. K., Riedel, A. R., Cruz, K. L., et al. 2016, ApJS, 225, 10

Famaey, B., Siebert, A., \& Jorissen, A. 2008, A\&A, 483, 453

Feigelson, E. D., Broos, P., Gaffney, J. A., III, et al. 2002, ApJ, 574, 258

Fernández, D., Figueras, F., \& Torra, J. 2008, A\&A, 480, 735

Fuhrmann, K. 2004, AN, 325, 3

Gagné, J., Faherty, J. K., Cruz, K., et al. 2014a, ApJL, 785, L14

Gagné, J., Lafrenière, D., Doyon, R., Malo, L., \& Artigau, É. 2014b, ApJ, 783,121

Gagné, J., Lafrenière, D., Doyon, R., et al. 2014c, ApJL, 792, L17

Gagné, J., Lafrenière, D., Doyon, R., et al. 2015, ApJ, 798, 73

Gaia Collaboration, Brown, A. G. A., Vallenari, A., et al. 2016, arXiv:1609. 04172

Gaidos, E. J. 1998, PASP, 110, 1259

Gatewood, G. 1995, ApJ, 445, 712

Gatewood, G., \& Coban, L. 2009, AJ, 137, 402

Gatewood, G., \& de Jonge, J. K. 1995, ApJ, 450, 364

Gatewood, G., de Jonge, K. J., \& Stephenson, B. 1993, PASP, 105, 1101

Girard, T. M., van Altena, W. F., Zacharias, N., et al. 2011, AJ, 142, 15

Gizis, J. E., Jao, W., Subasavage, J. P., \& Henry, T. J. 2007, ApJL, 669, L45

Gizis, J. E., Reid, I. N., \& Hawley, S. L. 2002, AJ, 123, 3356

Gliese, W., \& Jahreiß, H. 1991, Preliminary Version of the Third Catalogue of Nearby Stars, Tech. Rep., 1

Goldman, B., Röser, S., Schilbach, E., et al. 2013, A\&A, 559, A43

Gontcharov, G. A. 2006, A\&AT, 25, 145

Gray, R. O., Corbally, C. J., Garrison, R. F., et al. 2006, AJ, 132, 161

Guenther, E. W., Esposito, M., Mundt, R., et al. 2007, A\&A, 467, 1147

Guillout, P., Klutsch, A., Frasca, A., et al. 2009, A\&A, 504, 829

Henden, A. A., Levine, S., Terrell, D., \& Welch, D. L. 2015, in American Astronomical Society Meeting 225 Abstracts, 336.16

Henden, A. A., Templeton, M., Terrell, D., et al. 2016, yCat, 2336

Henry, T. J., Jao, W., Subasavage, J. P., et al. 2006, AJ, 132, 2360

Henry, T. J., Walkowicz, L. M., Barto, T. C., \& Golimowski, D. A. 2002, AJ, 123, 2002

Hillenbrand, L. A., \& Baliber, n. 2015, IAUGA, 22, 53943

Hillenbrand, L. A., Hoffer, A. S., \& Herczeg, G. J. 2013, AJ, 146, 85

Hinkley, S., Pueyo, L., Faherty, J. K., et al. 2013, ApJ, 779, 153

Hoffleit, D., \& Jaschek, C. 1991, The Bright star Catalogue (New Haven, CT: Yale Univ.)

Høg, E., Fabricius, C., Makarov, V. V., et al. 2000, A\&A, 355, L27

Houk, N. 1978, Michigan Catalogue of Two-dimensional Spectral Types for the HD Stars (Ann Arbor: Dept. of Astronomy, University of Michigan: distributed by University Microfilms International)

Houk, N. 1982, Michigan Catalogue of Two-dimensional Spectral Types for the HD stars, Vol. 3, Declinations -40_0 to -26_0 (Ann Arbor, MI: Department of Astronomy, University of Michigan) $12+390$

Houk, N., \& Cowley, A. P. 1975, University of Michigan Catalogue of twodimensional spectral types for the HD stars, Vol. I, Declinations -90_ to 
-53_0 (Ann Arbor, MI: Department of Astronomy, University of Michigan) $19+452$

Hunter, J. D. 2007, CSE, 9, 90

Jameson, R. F., Casewell, S. L., Bannister, N. P., et al. 2008, MNRAS, 384, 1399

Janson, M., Jayawardhana, R., Girard, J. H., et al. 2012, ApJL, 758, L2

Jao, W., Henry, T. J., Subasavage, J. P., et al. 2005, AJ, 129, 1954

Jeffries, R. D. 1995, MNRAS, 273, 559

Johnson, D. R. H., \& Soderblom, D. R. 1987, AJ, 93, 864

Jones, E., Oliphant, T., Peterson, P., et al. 2001, SciPy: Open source scientific tools for Python, http://www.scipy.org/

Kastner, J. H., Thompson, E. A., Montez, R., et al. 2012, ApJL, 747, L23

Kastner, J. H., Zuckerman, B., Weintraub, D. A., \& Forveille, T. 1997, Sci, 277, 67

Kharchenko, N. V., Piskunov, A. E., Schilbach, E., Röser, S., \& Scholz, R.-D. 2013, A\&A, 558, A53

Kharchenko, N. V., Scholz, R.-D., Piskunov, A. E., Röser, S., \& Schilbach, E. 2007, AN, 328, 889

King, J. R., Villarreal, A. R., Soderblom, D. R., Gulliver, A. F., \& Adelman, S. J. 2003, AJ, 125, 1980

Kirkpatrick, J. D., Cruz, K. L., Barman, T. S., et al. 2008, ApJ, 689, 1295

Kirkpatrick, J. D., Henry, T. J., \& McCarthy, D. W., Jr. 1991, ApJS, 77, 417

Kiss, L. L., Moór, A., Szalai, T., et al. 2011, MNRAS, 411, 117

Klutsch, A., Freire Ferrero, R., Guillout, P., et al. 2014, A\&A, 567, A52

Kordopatis, G., Gilmore, G., Steinmetz, M., et al. 2013, AJ, 146, 134

Kraus, A. L., \& Hillenbrand, L. A. 2007, AJ, 134, 2340

Kraus, A. L., Ireland, M. J., Cieza, L. A., et al. 2014a, ApJ, 781, 20

Kraus, A. L., Shkolnik, E. L., Allers, K. N., \& Liu, M. C. 2014b, AJ, 147, 146

Lépine, S., \& Shara, M. M. 2005, AJ, 129, 1483

Lépine, S., \& Simon, M. 2009, AJ, 137, 3632

Liu, M. C., Dupuy, T. J., \& Allers, K. N. 2013a, AN, 334, 85

Liu, M. C., Magnier, E. A., Deacon, N. R., et al. 2013b, ApJL, 777, L20

López-Santiago, J., Albacete Colombo, J. F., \& López-García, M. A. 2010a, A\&A, 524, A97

López-Santiago, J., Micela, G., \& Montes, D. 2009, A\&A, 499, 129

López-Santiago, J., Montes, D., Crespo-Chacón, I., \& Fernández-Figueroa, M. J. 2006, ApJ, 643, 1160

López-Santiago, J., Montes, D., Gálvez-Ortiz, M. C., et al. 2010b, A\&A, 514, A97

Luhman, K. L., Stauffer, J. R., \& Mamajek, E. E. 2005, ApJL, 628, L69

Lurie, J. C., Henry, T. J., Jao, W.-C., et al. 2014, AJ, 148, 91

Lyo, A.-R., Lawson, W. A., \& Bessell, M. S. 2004, MNRAS, 355, 363

Makarov, V. V. 2007, ApJS, 169, 105

Makarov, V. V., Olling, R. P., \& Teuben, P. J. 2004, MNRAS, 352, 1199

Makarov, V. V., \& Urban, S. 2000, MNRAS, 317, 289

Maldonado, J., Martínez-Arnáiz, R. M., Eiroa, C., Montes, D., \& Montesinos, B. 2010, A\&A, 521, A12

Malo, L., Artigau, É., Doyon, R., et al. 2014a, ApJ, 788, 81

Malo, L., Doyon, R., Feiden, G. A., et al. 2014b, ApJ, 792, 37

Malo, L., Doyon, R., Lafrenière, D., et al. 2013, ApJ, 762, 88

Mamajek, E. E. 2005, ApJ, 634, 1385

Mamajek, E. E. 2007, in IAU Symp. 237, Triggered Star Formation in a Turbulent ISM, ed. B. G. Elmegreen \& J. Palous (Cambridge: Cambridge Univ. Press), 442

Mamajek, E. E. 2015, arXiv:1507.06697

Mamajek, E. E. 2016, in IAU Symp. 314, Young Stars \& Planets Near the Sun, ed. J. H. Kastner, B. Stelzer, \& S. A. Metchev (Cambridge: Cambridge Univ. Press), 21

Mamajek, E. E., Bartlett, J. L., Seifahrt, A., et al. 2013, AJ, 146, 154

Mamajek, E. E., \& Bell, C. P. M. 2014, MNRAS, 445, 2169

Mamajek, E. E., \& Hillenbrand, L. A. 2008, ApJ, 687, 1264

Mamajek, E. E., Lawson, W. A., \& Feigelson, E. D. 1999, ApJL, 516 L77

Mamajek, E. E., Lawson, W. A., \& Feigelson, E. D. 2000, ApJ, 544, 356

Marocco, F., Andrei, A. H., Smart, R. L., et al. 2013, AJ, 146, 161

Marois, C., Macintosh, B., Barman, T., et al. 2008, Sci, 322, 1348

Mason, B. D., Wycoff, G. L., Hartkopf, W. I., Douglass, G. G., \& Worley, C. E. 2015, yCat, 1, 2026

Mathieu, R. D., Baraffe, I., Simon, M., Stassun, K. G., \& White, R. 2007, in Protostars and Planets V, Dynamical Mass Measurements of Pre-MainSequence Stars: Fundamental Tests of the Physics of Young Stars, ed. B. Reipurth, D. Jewitt, \& K. Keil (Tucson, AZ: Univ. Arizona Press), 411 McArthur, B. E., Endl, M., Cochran, W. D., et al. 2004, ApJL, 614, L81

McCarthy, K., \& White, R. J. 2012, AJ, 143, 134

McCarthy, K., \& Wilhelm, R. J. 2014, AJ, 148, 70
Mentuch, E., Brandeker, A., van Kerkwijk, M. H., Jayawardhana, R., \& Hauschildt, P. H. 2008, ApJ, 689, 1127

Messina, S., Desidera, S., Lanzafame, A. C., Turatto, M., \& Guinan, E. F. 2011, A\&A, 532, A10

Micela, G., Sciortino, S., Harnden, F. R., Jr., et al. 1999, A\&A, 341, 751

Mishenina, T. V., Soubiran, C., Kovtyukh, V. V., Katsova, M. M., \& Livshits, M. A. 2012, A\&A, 547, A106

Montes, D., López-Santiago, J., Fernández-Figueroa, M. J., \& Gálvez, M. C. 2001a, A\&A, 379, 976

Montes, D., López-Santiago, J., Gálvez, M. C., et al. 2001b, MNRAS, 328,45

Moór, A., Ábrahám, P., Derekas, A., et al. 2006, ApJ, 644, 525

Moór, A., Pascucci, I., Kóspál, Á., et al. 2011, ApJS, 193, 4

Moór, A., Szabó, G. M., Kiss, L. L., et al. 2013, MNRAS, 435, 1376

Murphy, S. J. 2012, PhD thesis, Mount Stromlo Observatory, Australian National Univ.

Murphy, S. J., \& Lawson, W. A. 2015, MNRAS, 447, 1267

Murphy, S. J., Lawson, W. A., \& Bessell, M. S. 2010, MNRAS, 406, L50

Murphy, S. J., Lawson, W. A., \& Bessell, M. S. 2013, MNRAS, 435, 1325

Nakajima, T., \& Morino, J.-I. 2012, AJ, 143, 2

Nakajima, T., Morino, J.-I., \& Fukagawa, M. 2010, AJ, 140, 713

Naud, M.-E., Artigau, É., Malo, L., et al. 2014, ApJ, 787, 5

Nidever, D. L., Marcy, G. W., Butler, R. P., Fischer, D. A., \& Vogt, S. S. 2002, ApJS, 141, 503

Nordström, B., Mayor, M., Andersen, J., et al. 2004, A\&A, 418, 989

Ortega, V. G., Jilinski, E., de La Reza, R., \& Bazzanella, B. 2007, MNRAS, 377, 441

Platais, I., Melo, C., Mermilliod, J., et al. 2007, A\&A, 461, 509

Pöhnl, H., \& Paunzen, E. 2010, A\&A, 514, A81

Pourbaix, D., Tokovinin, A. A., Batten, A. H., et al. 2004, A\&A, 424, 727

Preibisch, T., \& Mamajek, E. 2008, Handbook of Star Forming Regions, Vol. 2 (San Francisco, CA: ASP)

Raghavan, D., McAlister, H. A., Henry, T. J., et al. 2010, ApJS, 190, 1

Rebolo, R., Martin, E. L., \& Magazzu, A. 1992, ApJL, 389, L83

Reid, I. N., Hawley, S. L., \& Gizis, J. E. 1995, AJ, 110, 1838

Ribas, I. 2003, A\&A, 400, 297

Rice, E. L., Faherty, J. K., \& Cruz, K. L. 2010, ApJL, 715, L165

Riedel, A. 2016a, LACEwING Moving Group Identification Code, Zenodo, doi:10.5281/zenodo.199161

Riedel, A., Blunt, S., \& Lambrides, E. 2016a, ariedel/young_catalog: The Catalog of Suspected Nearby Young Stars (2016.1118), Zenodo, doi:10. 5281 /zenodo. 201437

Riedel, A. R. 2016b, in IAU Symp. 314, Young Stars \& Planets Near the Sun, ed. J. H. Kastner, B. Stelzer, \& S. A. Metchev (Cambridge: Cambridge Univ. Press), 33

Riedel, A. R. 2016c, LACEwING: LocAting Constituent mEmbers In Nearby Groups, Astrophysics Source Code Library, ascl:1601.011

Riedel, A. R., Alam, M. K., Rice, E. L., Cruz, K. L., \& Henry, T. J. 2016b, arXiv: 1610.03867

Riedel, A. R., Finch, C. T., Henry, T. J., et al. 2014, AJ, 147, 85

Riedel, A. R., Murphy, S. J., Henry, T. J., et al. 2011, AJ, 142, 104

Riedel, A. R., Subasavage, J. P., Finch, C. T., et al. 2010, AJ, 140, 897

Robin, A. C., Reylé, C., Derrière, S., \& Picaud, S. 2003, A\&A, 409, 523

Rodriguez, D. R., Bessell, M. S., Zuckerman, B., \& Kastner, J. H. 2011, ApJ, 727, 62

Rodriguez, D. R., Zuckerman, B., Faherty, J. K., \& Vican, L. 2014, A\&A, 567, A20

Rodriguez, D. R., Zuckerman, B., Kastner, J. H., et al. 2013, ApJ, 774, 101

Röser, S., Demleitner, M., \& Schilbach, E. 2010, AJ, 139, 2440

Röser, S., Schilbach, E., Piskunov, A. E., Kharchenko, N. V., \& Scholz, R.-D. 2011, A\&A, 531, A92

Rucinski, S. M., \& Krautter, J. 1983, A\&A, 121, 217

Schlieder, J. E., Lépine, S., Rice, E., et al. 2012a, AJ, 143, 114

Schlieder, J. E., Lépine, S., \& Simon, M. 2010, AJ, 140, 119

Schlieder, J. E., Lépine, S., \& Simon, M. 2012b, AJ, 143, 80

Schlieder, J. E., Lépine, S., \& Simon, M. 2012c, AJ, 144, 109

Schmitt, J. H. M. M., Fleming, T. A., \& Giampapa, M. S. 1995, ApJ, 450, 392

Schneider, A., Melis, C., \& Song, I. 2012a, ApJ, 754, 39

Schneider, A., Song, I., Melis, C., et al. 2013, ApJ, 777, 78

Schneider, A., Song, I., Melis, C., Zuckerman, B., \& Bessell, M. 2012b, ApJ, 757,163

Schneider, A. C., Cushing, M. C., Kirkpatrick, J. D., et al. 2014, AJ, 147, 34

Scholz, A., Coffey, J., Brandeker, A., \& Jayawardhana, R. 2007, ApJ, 662,1254

Shaya, E. J., \& Olling, R. P. 2011, ApJS, 192, 2 
Shkolnik, E., Liu, M. C., \& Reid, I. N. 2009, ApJ, 699, 649

Shkolnik, E. L., Anglada-Escudé, G., Liu, M. C., et al. 2012, ApJ, 758, 56

Shkolnik, E. L., Hebb, L., Liu, M. C., Reid, I. N., \& Collier Cameron, A. 2010 ApJ, 716, 1522

Shkolnik, E. L., Liu, M. C., Reid, I. N., Dupuy, T., \& Weinberger, A. J. 2011, ApJ, 727, 6

Skrutskie, M. F., Cutri, R. M., Stiening, R., et al. 2006, AJ, 131, 1163

Skuljan, J., Hearnshaw, J. B., \& Cottrell, P. L. 1999, MNRAS, 308, 731

Smart, R. L., Ioannidis, G., Jones, H. R. A., Bucciarelli, B., \& Lattanzi, M. G. 2010, A\&A, 514, A84

Soderblom, D. R. 2010, ARA\&A, 48, 581

Soderblom, D. R., \& Mayor, M. 1993, AJ, 105, 226

Soderblom, D. R., Nelan, E., Benedict, G. F., et al. 2005, AJ, 129, 1616

Söderhjelm, S. 1999, A\&A, 341, 121

Song, I., Bessell, M. S., \& Zuckerman, B. 2002, ApJL, 581, L43

Song, I., Zuckerman, B., \& Bessell, M. S. 2003, ApJ, 599, 342

Stauffer, J., Tanner, A. M., Bryden, G., et al. 2010, PASP, 122, 885

Stauffer, J. R., Hartmann, L. W., Fazio, G. G., et al. 2007, ApJS, 172, 663

Subasavage, J. P., Jao, W., Henry, T. J., et al. 2009, AJ, 137, 4547

Tabernero, H. M., Montes, D., \& González Hernández, J. I. 2012, A\&A, 547, A13

Teixeira, R., Ducourant, C., Chauvin, G., et al. 2008, A\&A, 489, 825

Teixeira, R., Ducourant, C., Chauvin, G., et al. 2009, A\&A, 503, 281

Thorstensen, J. R., \& Kirkpatrick, J. D. 2003, PASP, 115, 1207

Torres, C. A. O., da Silva, L., Quast, G. R., de la Reza, R., \& Jilinski, E. 2000, AJ, 120,1410

Torres, C. A. O., Quast, G. R., da Silva, L., et al. 2006, A\&A, 460, 695

Torres, C. A. O., Quast, G. R., de La Reza, R., et al. 2003, in Open Issues in Local Star Formation, Vol. 299 ed. J. Lépine \& J. Gregorio-Hetem (Dordecht: Kluwer), 83

Torres, C. A. O., Quast, G. R., Melo, C. H. F., \& Sterzik, M. F. 2008, Handbook of Star Forming Regions, Vol. 2 (San Francisco, CA: ASP)

Torres, C. A. O., Quast, G. R., \& Montes, D. 2016, in IAU Symp. 314, Young Stars \& Planets Near the Sun, ed. J. H. Kastner, B. Stelzer, \& S. A. Metchev (Cambridge: Cambridge Univ. Press), 77
Torres, G., \& Ribas, I. 2002, ApJ, 567, 1140

Valenti, J. A., \& Fischer, D. A. 2005, ApJS, 159, 141

van Altena, W. F., Lee, J. T., \& Hoffleit, E. D. 1995, The General Catalogue of Trigonometric [stellar] Parallaxes (4th ed.; New Haven, CT: Yale Univ. Press)

van den Ancker, M. E., Pérez, M. R., de Winter, D., \& McCollum, B. 2000, A\&A, 363, L25

van Leeuwen, F. 2007, A\&A, 474, 653

van Leeuwen, F. 2009, A\&A, 497, 209

Voges, W., Aschenbach, B., Boller, T., et al. 1999, A\&A, 349, 389

Voges, W., Aschenbach, B., Boller, T., et al. 2000, IAUC, 7432

von Braun, K., Boyajian, T. S., Kane, S. R., et al. 2011, ApJL, 729, L26

Vrba, F. J., Henden, A. A., Luginbuhl, C. B., et al. 2004, AJ, 127, 2948

Wahhaj, Z., Liu, M. C., Biller, B. A., et al. 2011, ApJ, 729, 139

Walt, S. v. d., Colbert, S. C., \& Varoquaux, G. 2011, CSE, 13, 22

Webb, R. A., Zuckerman, B., Platais, I., et al. 1999, ApJL, 512, L63

Weinberger, A. J., Anglada-Escudé, G., \& Boss, A. P. 2013, ApJ, 762, 118

Weis, E. W., Lee, J. T., Lee, A. H., et al. 1999, AJ, 117, 1037

West, A. A., Hawley, S. L., Bochanski, J. J., et al. 2008, AJ, 135, 785

Wilson, R. E. 1953, General catalogue of stellar radial velocities (Washington, D.C.: Carnegie Institution of Washington)

Xing, L.-F., \& Xing, Q.-F. 2012, A\&A, 537, A91

Yee, J. C., \& Jensen, E. L. N. 2010, ApJ, 711, 303

Zacharias, N., Finch, C. T., Girard, T. M., et al. 2013, AJ, 145, 44

Zapatero Osorio, M. R., Béjar, V. J. S., Miles-Páez, P. A., et al. 2014, A\&A, 568, A6

Zuckerman, B. 2001, in ASP Conf. Ser. 244, Young Stars Near Earth: Progress and Prospects, ed. R. Jayawardhana \& T. Greene (San Francisco, CA: ASP), 122

Zuckerman, B., Bessell, M. S., Song, I., \& Kim, S. 2006, ApJL, 649, L115

Zuckerman, B., Rhee, J. H., Song, I., \& Bessell, M. S. 2011, ApJ, 732, 61

Zuckerman, B., \& Song, I. 2004, ARA\&A, 42, 685

Zuckerman, B., Song, I., \& Bessell, M. S. 2004, ApJL, 613, L65

Zuckerman, B., Song, I., \& Webb, R. A. 2001, ApJ, 559, 388

Zuckerman, B., Vican, L., Song, I., \& Schneider, A. 2013, ApJ, 778, 5 